[99] J. Xu. A Theory of Types and Type Inference in Logic Programming Languages. PhD thesisTSUNY at Stony BrookГ1989.

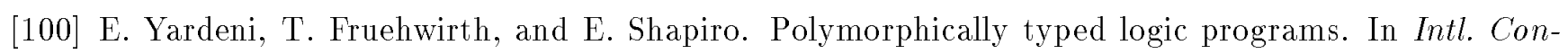
ference on Logic Programming ParisTFranceГJune 1991.

[101] E. Yardeni and E. Shapiro. A type system for logic programs. In E. ShapiroTeditorT Concurrent Prolog Tvolume 2. MIT Press 1987.

[102] C. Zaniolo. The database language GEM. In ACM SIGMOD Conference on Management of Data pages $423-434 \Gamma 1983$.

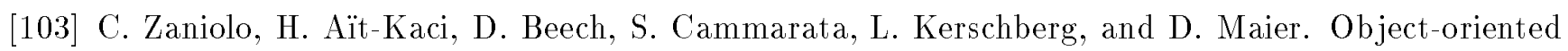
database and knowledge systems. Technical Report DB-038-85ГМССГ1985. 
[83] R. Ramakrishnan. Magic Templates: A spellbinding approach to logic programs. In IEEE Symposium on Logic Programming Tpages 140-159Г1988.

[84] K.A. Ross. Relations with relation names as arguments: Algebra and calculus. In ACM SIGACTSIGMOD-SIGART Symposium on Principles of Database SystemsTMay 1992.

[85] M.A. RothГH.F. KorthГand A. Silberschatz. Extended algebra and calculus for $\neg 1 \mathrm{NF}$ relational databases. Technical Report 84-36 UUniv. of Texas at AustinГ1985.

[86] J.W. Schmidt. Some high-level language constructs for data of type relation. ACM Transactions on Database SystemsГ2(3):247-261ГSeptember 1977.

[87] D.W. Shipman. The functional data model and the data language DAPLEX. ACM Transactions on Database Systems pages 140-173Г1981.

[88] M. Stefik and D.G. Bobrow. Object-oriented programming: Themes and variations. The AI MagazineTpages 40-62ГJanuary 1986.

[89] B. Stroustrup. The C++ Programming Language. Addison-WesleyГReadingГМАГ1986.

[90] K. Thirunarayan and M. Kifer. A theory of nonmonotonic inheritance based on annotated logic. Artificial IntelligenceГ60(1):23-50ГMarch 1993.

[91] D.S. Touretzky. The Mathematics of Inheritance. Morgan-KaufmannГLos AltosГCAГ1986.

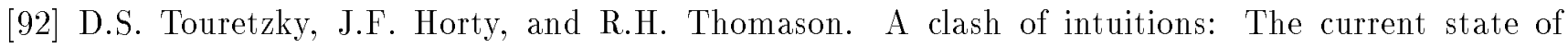
nonmonotonic multiple inheritance systems. In Intl. Joint Conference on Artificial Intelligence $\Gamma$ pages $476-482 \Gamma 1987$.

[93] J.D. Ullman. Database theory: Past and future. In ACM SIGACT-SIGMOD-SIGART Symposium on Principles of Database SystemsTpages 1-10Г1987.

[94] J.F. Ullman. Principles of Database and Knowledge-Base Systems, Volume 1. Computer Science Press 1988.

[95] A. Van Gelder. The alternating fixpoint of logic programs with negation. In ACM SIGACTSIGMOD-SIGART Symposium on Principles of Database SystemsГpages 1-10Г1989.

[96] A. Van GelderTK.A. RossTand J.S. Schlipf. The well-founded semantics for general logic programs. In ACM SIGACT-SIGMOD-SIGART Symposium on Principles of Database SystemsГpages 221$230 \Gamma 1988$.

[97] P. Wegner. The object-oriented classification paradigm. In B. Shriver and P. WegnerTeditors Research Directions in Object-Oriented ProgrammingГpages 479-560. MIT PressГ1987.

[98] J. Wu. A Theory of Types and Polymorphism in Logic Programming. PhD thesisTSUNY at Stony BrookГ1992. 


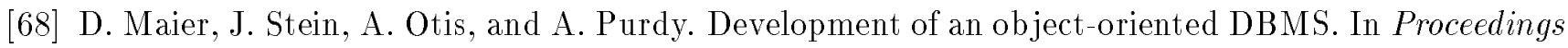
of OOPSLA-86Гpages 472-482Г1986.

[69] J. McCarthy. First order theories of individual concepts and propositions. In J.E. Hayes and D. Michie $\Gamma$ editors $\Gamma$ Machine Inteligence $\Gamma$ volume $9 \Gamma$ pages 129-147. Edinburgh University Press $\Gamma$ 1979.

[70] B. Meyer. Object-Oriented Software Construction. Prentice HallГ1988.

[71] D. Miller. A logical analysis of modules in logic programming. Journal of Logic Programming $\Gamma$ 6:79-108Г1989.

[72] M. Minsky. A framework for representing knowledge. In J. HaugelandTeditor $\Gamma$ Mind design $\Gamma$ pages 95-128. MIT PressГCambridgeГМАГ1981.

[73] P. Mishra. Towards a theory of types in Prolog. In IEEE Symposium on Logic Programming Tpages $289-298 \Gamma 1984$.

[74] J.C. Mitchell. Toward a typed foundation for method specialization and inheritance. In $A C M$ Symposium on Principles of Programming Languages $\Gamma$ pages 109-124Г1990.

[75] K. MorrisTJ. NaughtonTY. SaraiyaГJ. UllmanTand A. Van Gelder. YAWN! (Yet another window on NAIL!). IEEE Database EngineeringГ6:211-226Г1987.

[76] A. Motro. BAROQUE: A browser for relational databases. ACM Trans. on Office Information Systems $\Gamma 4(2): 164-181 \Gamma 1986$.

[77] S. Naqvi and S. Tsur. A Logical Language for Data and Knowledge Bases. Computer Science Press 1989.

[78] E. Neuhold and M. Stonebraker. Future directions in DBMS research (The Laguna Beech report). SIGMOD Record $18(1)$ TMarch 1989.

[79] G. PhippsГM.A. DerrTand K.A. Ross. Glue-Nail: A deductive database system. In ACM SIGMOD Conference on Management of DataГpages 308-317Г1991.

[80] H. Przymusinska and M. Gelfond. Inheritance hierarchies and autoepistemic logic. In Intl. Symposium on Methodologies for Intelligent Systems 1989.

[81] T.C. Przymusinski. On the declarative semantics of deductive databases and logic programs. In

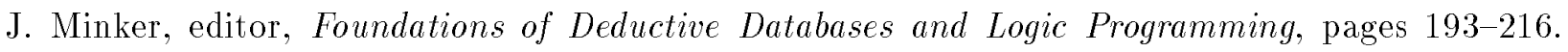
Morgan KaufmannTLos AltosГCАГ1988.

[82] T.C. Przymusinski. Every logic program has a natural stratification and an iterated least fixed point model. In ACM SIGACT-SIGMOD-SIGART Symposium on Principles of Database Systems $\Gamma$ pages 11-21Г1989. 
[52] M. Kifer and J. Wu. A logic for programming with complex objects. Journal of Computer and System SciencesГ46Г1993. A special issue from PODS-89. To appear.

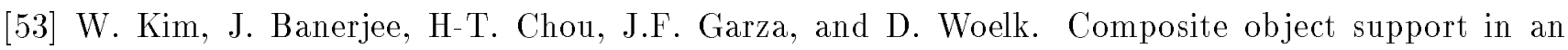
object-oriented database system. In Proceedings of OOPSLA-87Г1987.

[54] P.G. Kolaitis and C.H. Papadimitriou. Why not negation by fixpoint. In ACM SIGACT-SIGMODSIGART Symposium on Principles of Database Systems $\Gamma$ pages 231-239Г1988.

[55] R. Krishnamurthy and S. Naqvi. Towards a real horn clause language. In Intl. Conference on Very Large Data BasesГ1988.

[56] T. KrishnaprasadT M. KiferTand D.S. Warren. On the circumscriptive semantics of inheritance networks. In Intl. Symposium on Methodologies for Intelligent Systems Гpages 448-457Г1989.

[57] T. KrishnaprasadГM. KiferTand D.S. Warren. On the declarative semantics of inheritance networks. In Intl. Joint Conference on Artificial IntelligenceГpages 1099-1103Г1989.

[58] G. Kuper and M.Y. Vardi. A new approach to database logic. In ACM SIGACT-SIGMOD-SIGART Symposium on Principles of Database SystemsГ1984.

[59] G.M. Kuper. An extension of LPS to arbitrary sets. Technical reportГ IBMГ Yorktown HeightsГ 1987.

[60] G.M. Kuper. Logic programming with sets. Journal of Computer and System Sciences $441(1): 44-64 \Gamma$ August 1990.

[61] E. LaenensTD. SaccaTand D. Vermeir. Extending logic programming. In ACM SIGMOD Conference on Management of DataГpages 184-193ГJune 1990.

[62] E. Laenens and D. Vermeir. A fixpoint semantics for ordered logic. Journal Logic and Computation $1(2): 159-185 \Gamma 1990$.

[63] C. Lecluse and P. Richard. The $\mathrm{O}_{2}$ database programming language. In Intl. Conference on Very Large Data BasesTAugust 1989.

[64] J.W. Lloyd. Foundations of Logic Programming (Second Edition). Springer VerlagГ1987.

[65] D. Maier. A logic for objects. In Workshop on Foundations of Deductive Databases and Logic Programming Гpages 6-26ГWashington D.C.CAugust 1986.

[66] D. Maier. Why database languages are a bad idea (position paper). In Proc. of the Workshop on Database Programming Languages TRoscoffTFranceГSeptember 1987.

[67] D. Maier. Why isn't there an object-oriented data model. Technical report Oregon Graduate CenterГMay 1989. 
[38] H. Geffner and T. Verma. Inheritance = chaining + defeat. In Intl. Symposium on Methodologies for Intelligent Systems $\Gamma$ pages 411-418Г1989.

[39] M. Gelfond and V. Lifschitz. The stable model semantics for logic programming. In Logic Programming: Proceedings of the Fifth Conference and Symposium Гpages 1070-1080Г1988.

[40] R.P. Hall. Computational approaches to analogical reasoning: A comparative study. Artificial Intelligence $\mathrm{30} 30: 39-120 \Gamma 1989$.

[41] P.J. Hayes. The logic for frames. In D. MetzingTeditorTFrame Conception and Text Understanding $\Gamma$ pages 46-61. Walter de Gruyter and Co.Г1979.

[42] P. Hill and R. Topor. A semantics for typed logic programs. In F. PfenningTeditor $\Gamma$ Types in Logic Programming pages 1-62. The MIT Press 1992.

[43] J.F. HortyГR.H. ThomasonTand D.S. Touretzky. A skeptical theory of inheritance in nonmonotonic semantic nets. In National Conference on Artificial Intelligence Tpages 358-363Г1987.

[44] R. Hull and M. Yoshikawa. ILOG: Declarative creation and manipulation of object identifiers. In

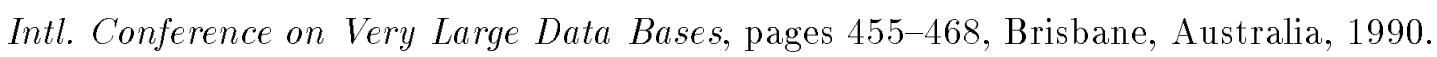

[45] F.N. Kesim and M. Sergot. On the evolution of objects in a logic programming framework. In Pro-

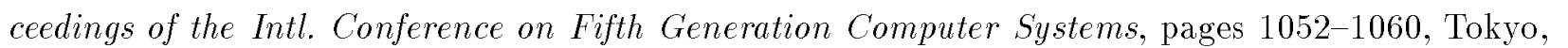
JapanTJune 1992.

[46] S.N. Khoshafian and G.P. Copeland. Object identity. In Proceedings of OOPSLA-86Гpages 406-416Г 1986.

[47] M. Kifer TW. Kim Tand Y. Sagiv. Querying object-oriented databases. In ACM SIGMOD Conference on Management of DataГpages 393-402ГJune 1992.

[48] M. Kifer and G. Lausen. F-logic: A higher-order language for reasoning about objectsTinheritance and schema. In ACM SIGMOD Conference on Management of Data Tpages 134-146Г1989.

[49] M. Kifer and G. Lausen. Behavioral inheritance in F-logic. in preparation 1993.

[50] M. Kifer and J. Wu. A logic for object-oriented logic programming (Maier's O-logic revisited). In ACM SIGACT-SIGMOD-SIGART Symposium on Principles of Database Systems $\Gamma$ pages 379-393Г March 1989.

[51] M. Kifer and J. Wu. A first-order theory of types and polymorphism in logic programming. In

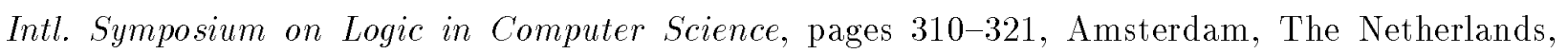
July 1991. Expanded version: TR 90/23 under the same title Department of Computer Science University at Stony BrookГJuly 1990. 
[22] G. Brewka. The logic of inheritance in frame systems. In Intl. Joint Conference on Artificial Intelligence $\Gamma$ pages $483-488 \Gamma 1987$.

[23] P. Buneman and R.E. Frankel. FQL - A functional query language. In ACM SIGMOD Conference on Management of DataГpages 52-58Г1979.

[24] P. Buneman and A. Ohori. Using powerdomains to generalize relational databases. Theoretical Computer ScienceГ1989.

[25] L. Cardelli. A semantics of multiple inheritance. Information and Computation $\mathrm{76}(2): 138-164 \Gamma$ February 1988.

[26] M. CareyГD. DeWittTand S. Vanderberg. A data model and query language for EXODUS. In ACM SIGMOD Conference on Management of DataГ1988.

[27] C.L. Chang and R.C.T. Lee. Symbolic Logic and Mechanical Theorem Proving. Academic PressT 1973.

[28] W. Chen. A theory of modules based on second-order logic. In IEEE Symposium on Logic Programming $\Gamma$ pages 24-33ГSeptember 1987.

[29] W. Chen and M. Kifer. SortsTtypes and polymorphism in higher-order logic programming. Technical Report 92-CSE-7ГDepartment of Computer Science and Engineering $\Gamma$ Southern Methodist UniversityГMarch 1992.

[30] W. Chen TM. KiferTand D.S. Warren. HiLog: A foundation for higher-order logic programming. Journal of Logic ProgrammingГ15(3):187-230ГFebruary 1993.

[31] W. Chen and D.S. Warren. C-logic for complex objects. In ACM SIGACT-SIGMOD-SIGART Symposium on Principles of Database SystemsГpages 369-378ГMarch 1989.

[32] W.F. Clocksin and C.S. Mellish. Programming in Prolog. Springer VerlagT1981.

[33] W.R. CookTW.L. HillГ and P.S. Canning. Inheritance is not subtyping. In ACM Symposium on Principles of Programming Languages Tpages 125-136Г1990.

[34] G. Dobbie and R. Topor. A model for inheritance and overriding in deductive object-oriented systems. In Sixteenth Australian Computer Science ConferenceГJanuary 1993.

[35] H.B. Enderton. A Mathematical Introduction to Logic. Academic PressГ1972.

[36] D.W. Etherington and R. Reiter. On inheritance hierarchies with exceptions. In National Conference on Artificial IntelligenceГpages 104-108ГWashingtonГD.C.Г1983.

[37] R. Fikes and T. Kehler. The role of frame-based representation in reasoning. Communications of А СМГ28(9):904-920Г1985. 
[8] R. Anderson and W.W. Bledsoe. A linear format resolution with merging and a new technique for establishing completeness. Journal of ACMГ17(3):525-534Г1970.

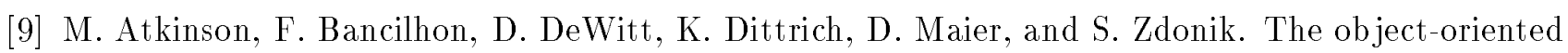
database system manifesto. In Intl. Conference on Deductive and Object-Oriented DatabasesTpages $40-57 \Gamma 1989$.

[10] M. Balaban. The generalized concept formalism - A frames and logic based representation model. In Proceedings of the Canadian Artificial Intelligence Conference Tpages 215-219Г1986.

[11] M. Balaban and S. Strack. LOGSTER - A relationalГ object-oriented system for knowledge representation. In Intl. Symposium on Methodologies for Intelligent Systems Гpages 210-219Г1988.

[12] F. Bancilhon. Object-oriented database systems. In ACM SIGACT-SIGMOD-SIGART Symposium on Principles of Database Systems Tpages 152-162Г1988.

[13] F. Bancilhon and S.N. Khoshafian. A calculus of complex objects. Journal of Computer and System SciencesГ38(2):326-340ГApril 1989.

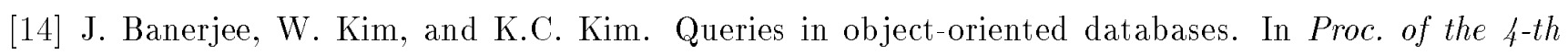
Intl. Conf. on Data Engineering LLos AngelesГCАГFebruary 1988.

[15] C. Beeri. Formal models for object-oriented databases. In Intl. Conference on Deductive and Object-Oriented DatabasesГpages 370-395. Elsevier Science Pub1.Г1989.

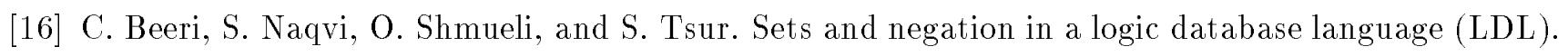
Technical reportГМССГ1987.

[17] C. BeeriT R. NasrTand S. Tsur. Embedding $\psi$-terms in a horn-clause logic language. In Third International Conference on Data and Knowledge Bases: Improving Usability and Responsiveness $\Gamma$ pages 347-359. Morgan KaufmannГ1988.

[18] C. Beeri and R. Ramakrishnan. On the power of magic. Journal of Logic Programming $\Gamma 10: 255-300 \Gamma$ April 1991.

[19] A.J. Bonner and M. Kifer. Transaction logic programming (or a logic of declarative and procedural knowledge). Technical Report CSRI-270Г University of TorontoГ April 1992. Revised: April 1993. Available in csri-technical-reports/270/report.ps by anonymous ftp to csri.toronto.edu.

[20] A.J. Bonner and M. Kifer. Transaction logic programming. In Intl. Conference on Logic Programming $\mathrm{Budapest \Gamma Hungary \Gamma} \mathrm{June} \mathrm{1993.} \mathrm{To} \mathrm{appear.}$

[21] S. Brass and U.W. Lipeck. Semantics of inheritance in logical object specifications. In Intl. Conference on Deductive and Object-Oriented DatabasesTpages 411-430ГDecember 1991. 
1. Unification of sets:

This is not a problem specific to F-logic butTratherTis a fact of life. Every language that allows the use of sets in any essential way has to put up with the exponential worst-case complexity of set-unification.

\section{Permutation of methods inside molecules:}

Since methods may be denoted by nonground id-terms $T$ they can match each other in many different ways. For instance $\Gamma$ in unifying $P[X \rightarrow V ; Y \rightarrow W]$ and $P\left[X^{\prime} \rightarrow V^{\prime} ; Y^{\prime} \rightarrow W^{\prime}\right] \Gamma$ the method denoted by $X$ can match either $X^{\prime}$ or $Y^{\prime}$; similarly for $Y$. Thus $\Gamma$ an extra complexity is expected due to the higher-order syntax of F-logic.

The key factor in estimating the complexity of unification in "practical" cases is the number of atoms comprising each molecule in the bodies of the rules. For $\Gamma$ if $n_{1}$ is the number of atoms in $T_{1}$ and $n_{2}$ is the same for $T_{2}$ Tthen the number of unifiers of $T_{1}$ into $T_{2}$ is bounded by $n_{2}^{n_{1}}$. Now $\Gamma$ to resolve a pair of

rules $\ldots \leftarrow \ldots, T_{1}, \ldots$ and $T_{2} \leftarrow \ldots \Gamma$ where $T_{1}$ and $T_{2}$ are data or signature molecules $\Gamma$ we need to unify $T_{1}$ into $T_{2}$ and $\Gamma$ therefore $\Gamma$ the above parameter $\Gamma n_{1} \Gamma$ which denotes the number of atoms in $T_{1} \Gamma$ is most crucial. HoweverTour experience with writing F-programs and all the examples in this paper show that this number is usually very small $(\leq 2)$ and soTwe believe Combinatorial explosion is unlikely to happen in practice.

\section{References}

[1] S. Abiteboul and C. Beeri. On the power of languages for manipulation of complex objects. Technical report TRapport de Recherche INRIAT1988. To appear in TODS.

[2] S. Abiteboul and S. Grumbach. COL: A logic-based language for complex objects. In Workshop on Database Programming LanguagesTpages 253-276ГRoscoffГFranceГSeptember 1987.

[3] S. Abiteboul and P.C. Kanellakis. Object identity as a query language primitive. In ACM SIGMOD Conference on Management of DataГpages 159-173Г1989.

[4] S. Abiteboul and V. Vianu. Procedural and declarative database update languages. In $A C M$ SIGACT-SIGMOD-SIGART Symposium on Principles of Database SystemsTpages 240-250Г1988.

[5] H. Ait-Kaci. An algebraic semantics approach to the effective resolution of type equations. Theoretical Computer ScienceГ45:293-351Г1986.

[6] H. Aït-Kaci and R. Nasr. LOGIN: A logic programming language with built-in inheritance. Journal of Logic ProgrammingГ3:185-215Г1986.

[7] H. Aït-Kaci and A. Podelski. Towards a meaning of LIFE. In Programming Languages Implementation and Logic Programming (PLILP'91) Tpages 255-274ГPassauTGermanyГAugust 1991. 
Input : Pair of molecules $\Gamma T_{1}$ and $T_{2}$.

Output : A complete set $\Omega$ of mgu's of $T_{1}$ into $T_{2}$.

1. If $i d\left(T_{1}\right)$ and $i d\left(T_{2}\right)$ are unifiableT then set $\theta:=U N I F Y\left(\left\langle i d\left(T_{1}\right)\right\rangle,\left\langle i d\left(T_{2}\right)\right\rangle\right)$.

Otherwise $\Gamma$ stop: $T_{1}$ and $T_{2}$ are not unifiable.

2. If $T_{1}$ is of the form $S[]$ (a degenerated molecule) Tthen stop: $\theta$ is the only mgu.

3. Set $\Omega:=\{\}$.

for each mapping $\lambda \in \operatorname{Maps}\left(T_{1}, T_{2}\right)$ do:

Set $\sigma_{\lambda}:=\theta$.

for each atom $\varphi$ in atoms $\left(T_{1}\right)$ do:

Let $\psi \stackrel{\text { def }}{=} \lambda(\varphi)$.

if $\operatorname{val}(\varphi)=\emptyset$ then Unify the tuples $\sigma_{\lambda}\left(\overrightarrow{S_{1}}\right)$ and $\sigma_{\lambda}\left(\overrightarrow{S_{2}}\right)$ Twhere

$\left.\overrightarrow{S_{1}}=<\operatorname{method}(\varphi), \arg _{1}(\varphi), \ldots, \arg _{n}(\varphi)\right\rangle$ and

$\overrightarrow{S_{2}}=<\operatorname{method}(\psi), \arg _{1}(\psi), \ldots, \arg _{n}(\psi)>$.

if $\sigma_{\lambda}\left(\overrightarrow{S_{1}}\right)$ and $\sigma_{\lambda}\left(\overrightarrow{S_{2}}\right)$ are unifiable then set $\sigma_{\lambda}:=\operatorname{UNIFY}\left(\sigma_{\lambda}\left(\overrightarrow{S_{1}}\right), \sigma_{\lambda}\left(\overrightarrow{S_{2}}\right)\right) \circ \sigma_{\lambda}$.

else discard this $\sigma_{\lambda}$ and jump out of the inner loop to select another $\lambda$.

else

Unify tuples $\sigma_{\lambda}\left(\overrightarrow{S_{1}}\right)$ and $\sigma_{\lambda}\left(\overrightarrow{S_{2}}\right)$ Twhere

$\left.\overrightarrow{S_{1}}=<\operatorname{method}(\varphi), \arg _{1}(\varphi), \ldots, \arg _{n}(\varphi), \operatorname{val}(\varphi)\right\rangle$ and

$\overrightarrow{S_{2}}=<\operatorname{method}(\psi), \arg _{1}(\psi), \ldots, \arg _{n}(\psi), \operatorname{val}(\psi)>$.

If $\sigma_{\lambda}\left(\overrightarrow{S_{1}}\right)$ and $\sigma_{\lambda}\left(\overrightarrow{S_{2}}\right)$ are unifiable then set $\sigma_{\lambda}:=\operatorname{UNIFY}\left(\sigma_{\lambda}\left(\overrightarrow{S_{1}}\right), \sigma_{\lambda}\left(\overrightarrow{S_{2}}\right)\right) \circ \sigma_{\lambda}$. else discard this $\sigma_{\lambda}$ and jump out of the inner loop to select another $\lambda$.

fi

fi

od

Set $\Omega:=\Omega \cup\left\{\sigma_{\lambda}\right\}$.

4. Return $\Omega \Gamma$ a complete set of mgu's of $T_{1}$ into $T_{2}$.

Figure 10: Computing a Complete Set of MGU's

Lemma B.1 The algorithm in Figure 10 correctly finds a complete set of mgu's of $T_{1}$ into $T_{2}$.

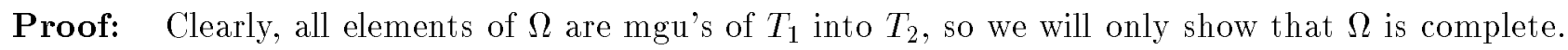
Consider a unifier $\theta$ of $T_{1}$ into $T_{2}$. By definition $\Gamma$ there is a mapping $\lambda \in \operatorname{Maps}\left(T_{1}, T_{2}\right)$ from atoms $\left(T_{1}\right)$ to atoms $\left(T_{2}\right)$ Tsuch that $\theta$ maps every $\varphi$ in atoms $\left(T_{1}\right)$ into an appropriate atom $\lambda(\varphi)$ in atoms $\left(T_{2}\right)$. Clearly substitution $\sigma_{\lambda}$ constructed in the inner for-loop in Step 3 of the above algorithm is a most general unifier that maps every $\varphi \in \operatorname{atoms}\left(T_{1}\right)$ to $\lambda(\varphi) \in \operatorname{atoms}\left(T_{2}\right)$. So $\mathrm{S} \theta$ is an instance of $\sigma_{\lambda}$.

Summarizing $\Gamma$ we have shown that 1 ) each element of $\Omega$ is an mgu; and 2 ) if $\theta$ is a unifier of $T_{1}$ into $T_{2} \Gamma$ then $\theta$ is an instance of an element $\sigma_{\lambda} \in \Omega$. Hence $\Gamma \Omega$ is a complete set of mgu's of $T_{1}$ into $T_{2}$.

In the worst-case Tthe algorithm of Figure 10 may yield an exponential number of unifiersTwhich may happen due to the following two factors: 


\section{Proposition A.6 Every locally stratified F-program has a unique perfect H-model.}

Proof: (Sketch) The proof is similar to that in [81]. AnotherTindirect proof can be derived from Theorem 17.1. Let $\Gamma\left(\mathbf{P}^{a}\right)$ be a translation of $\mathbf{P}^{a}$ into classical logic. To prove the result $\Gamma$ it is necessary to show that if $\prec_{\mathbf{P}^{a}}$ is well-founded then so is $\prec_{\Gamma\left(\mathbf{P}^{a}\right)} \Gamma$ where the latter is the order on ground atoms that is used in the usual definition of local stratification. Then Tit must be shown that (in the notation of Theorem 17.1) $\Phi$ maps $\ll_{\mathbf{P}^{a}}$-minimal models of $\mathbf{P}$ into $\ll_{\Gamma\left(\mathbf{P}^{a}\right)}$-minimal models of $\Gamma\left(\mathbf{P}^{a}\right)$.

\section{B Appendix: A Unification Algorithm for F-molecules}

This appendix presents an algorithm for finding a complete set of mgu's for a pair of molecules. We remind (Section 10.1) that all complete sets of mgu's are equivalent to each other and therefore it suffices to find just one such set. Recall that a molecule can be represented as a conjunction of its constituent atoms. For a pair of molecules $\Gamma T_{1}$ and $T_{2} \Gamma$ an mgu of $T_{1}$ into $T_{2}$ is a most general substitution that turns every constituent atom of $T_{1}$ into a constituent atom of $T_{2}$. Different correspondences between the constituent atoms of $T_{1}$ and $T_{2}$ may lead to different mgu's. To find a complete set of mgu's take all such correspondences into account. Let $\varphi$ be an atom of either of the following forms:

(i) $P\left[\right.$ Method $\left.@ Q_{1}, \ldots, Q_{k} \sim R\right]$ Twhere $\sim$ denotes any of the six

allowed types of arrows in method expressions;

(ii) $P\left[\right.$ Method $\left.@ Q_{1}, \ldots, Q_{k} \sim\{\}\right]$ Twhere $\sim$ denotes $\rightarrow$ or $\bullet ;$ or

(iii) $P\left[\right.$ Method $\left.@ Q_{1}, \ldots, Q_{k} \sim()\right]$ Twhere $\sim$ denotes $\Rightarrow$ or $\Rightarrow$.

The following notation is used in the unification algorithm in Figure 10:

$$
\begin{aligned}
& \operatorname{id}(\varphi)=P \Gamma \\
& \operatorname{method}(\varphi)=\text { Method } \Gamma \\
& \arg _{i}(\varphi)=Q_{i}, \text { for } i=1 \ldots k \Gamma \\
& \operatorname{val}(\varphi)= \begin{cases}R & \text { if } \varphi \text { is of the form (i) above } \\
\emptyset & \text { if } \varphi \text { has the form (ii) or (iii) above }\end{cases}
\end{aligned}
$$

In addition $\Gamma$ if $T$ is a molecule then $\operatorname{atoms}(T)$ will denote the set of atoms of $T$. If $T_{1}$ and $T_{2}$ are molecules then $\operatorname{Maps}\left(T_{1}, T_{2}\right)$ denotes the collection of mappings $\left\{\lambda: \operatorname{atoms}\left(T_{1}\right) \vdash \longrightarrow\right.$ atoms $\left.\left(T_{2}\right)\right\}$ that preserves method arities and the type of the method expression (or Tin other wordsTthe type of the arrow used in those expressions).

As noted in Section 10.1Tthe mgu of a pair of tuples of id-terms $\Gamma\left\langle P_{1}, \ldots, P_{n}\right\rangle$ and $\left\langle Q_{1}, \ldots, Q_{n}\right\rangle$ Tcoincides with the mgu of the terms $f\left(P_{1}, \ldots, P_{n}\right)$ and $f\left(Q_{1}, \ldots, Q_{n}\right)$ Twhere $f$ is an arbitrary $n$-ary function symbol. SoTwe can use any standard unification procedure to unify tuples of id-terms. Given a pair of tuples of id-terms $\overrightarrow{S_{1}}$ and $\overrightarrow{S_{2}}$ Twe use $U N I F Y\left(\overrightarrow{S_{1}}, \overrightarrow{S_{2}}\right)$ to denote a procedure that returns the mgu of $\overrightarrow{S_{1}}$ and $\overrightarrow{S_{2}}$ Tif one exists. 
Axioms of the IS-A hierarchy.

$$
\begin{array}{rll}
X:: Y & \leftarrow X:: Z \wedge Z:: Y & \text { \% Transitivity } \\
X \doteq Y & \leftarrow X:: Y \wedge Y:: X & \text { \% Acyclicity } \\
X: Y & \leftarrow X: Z \wedge Z:: Y & \text { \% Subclass inclusion }
\end{array}
$$

Axioms of typing. For every arity $n$ Tand for $\approx$ standing for either $\Rightarrow$ or $\Rightarrow$ :

$$
\begin{aligned}
& X\left[M @ A_{1}, \ldots, A_{n} \approx T\right] \leftarrow X^{\prime}\left[M @ A_{1}, \ldots, A_{n} \approx T\right] \wedge X:: X^{\prime} \\
& \% \text { Type inheritance } \\
& X\left[M @ A_{1}, \ldots A_{1}, \ldots, A_{n} \approx T\right] \leftarrow X\left[M @ A_{1}, \ldots, A_{i}^{\prime}, \ldots, A_{n} \approx T\right] \wedge A_{i}:: A_{i}^{\prime} \leftarrow \% \text { Input restriction } \\
& X\left[M @ A_{1}, \ldots, A_{n} \approx T\right] \leftarrow X\left[M @ A_{1}, \ldots, A_{n} \approx T^{\prime}\right] \wedge T^{\prime}:: T \\
& \% \text { Output relaxation }
\end{aligned}
$$

Axioms of scalarity. For every arity $n$ Tand for $\leadsto$ standing for $\rightarrow$ or $\bullet$ :

$$
\left(V \doteq V^{\prime}\right) \leftarrow X\left[M @ A_{1}, \ldots, A_{n} \sim V\right] \wedge X\left[M @ A_{1}, \ldots, A_{n} \sim V^{\prime}\right]
$$

Definition A.3 (Program Augmentation) For any programГP $\Gamma$ define its augmentation $\Gamma \mathbf{P}^{a} \Gamma$ to be the union of $\mathbf{P}$ with the relevant ground instances of the closure axioms.

A ground instance of an axiom is relevant if all atoms in its premises are relevant. An atom is relevant if and only if it occurs in the head of a rule in $\mathbf{P}^{*}$ or TrecursivelyCin the head of a axiom-instance whose relevance was established previously.

We can now rectify the earlier definitions by considering the order $\prec_{\mathbf{P}^{a}}$ instead of $\prec_{\mathbf{P}}$ Ti.e. Tby using augmentations of programs rather than programs themselves.

Definition A.4 (Locally Stratified Programs) An F-program $\mathbf{P}$ is locally stratified if the relation " $\prec_{\mathbf{P}^{a}}$ " is well-founded.

Definition A.5 (Perfect Models) H-models of $\mathbf{P}$ that are minimal with respect to $\ll_{\mathrm{p}^{a}}$ are called perfect models of $\mathbf{P}$.

Returning to programs $\mathbf{P}_{1}$ and $\mathbf{P}_{2}$ in (29) Twe can now see that they are not locally stratified with respect to Definition A.4 (while they were locally stratified with respect to Definition A.1). Indeed $\Gamma$ consider the following instance of the paramodulation axiom:

$$
p(b) \leftarrow p(a) \wedge(a \doteq b)
$$

Here $\Gamma$ both atoms in the premise are relevant. Therefore $\Gamma$ we have a negative cycle from $\neg p(b)$ through $p(a)$ Tand back to $p(b)$ in $\mathbf{D}_{\mathcal{L}}\left(\mathbf{P}_{1}^{a}\right)$; and from $\neg p(b)$ through $a \doteq b$ Tand back to $p(b)$ in $\mathbf{D}_{\mathcal{L}}\left(\mathbf{P}_{2}^{a}\right)$.

Similarly we can show that (31) has a negative cycle. Consider the following instance of the type inheritance axiom:

$$
b[a t t r \Rightarrow c] \leftarrow a[a t t r \Rightarrow c] \wedge b:: a
$$

This rule belongs to the augmentation of $\mathbf{P}$ because both of its premises are relevant. Thus $\Gamma \mathbf{D}_{\mathcal{L}}\left(\mathbf{P}_{3}^{a}\right)$ has the following negative cycle: $\neg b[$ attr $\Rightarrow c]$ Tto $p(a)$ Tto $a[$ attr $\Rightarrow c]$ Tand back to $b[$ attr $\Rightarrow c]$. 
The unique perfect model of $\mathbf{P}_{1}$ is $M_{1}=\{p(a), p(b), a \doteq b\}$ Tand $M_{2}=\{p(a), p(b)\}$ is the unique perfect model of $\mathbf{P}_{2}$. (These models are obtained from the definitions in [81] in a simple-minded way i.e. $\Gamma$ by considering equality as an ordinary predicate.) The trouble is that $p(b)$ is not supported by any rule. In fact $\Gamma$ in both programs $\Gamma p(b)$ was derived assuming $\neg p(b)$ is true.

As the above example shows $\mathrm{T}$ equality is a problem not only in F-logic but also in classical logic programming. However $\Gamma$ in the classical case $\Gamma$ this problem is side-stepped by disallowing equality to occur in the rule heads. Unfortunately this shortcut does not work for F-logic since equality may be derived even if it is not mentioned explicitly. For instance $\{a:: b, b:: a\} \models(a \doteq b)$ and $\{a[a t t r \rightarrow$ $b], a[a t t r \rightarrow c]\} \mid=(b \doteq c)$.

Another difficulty comes from the closure properties inherent in F-programs. For instanceTthe built-in property of signature inheritance corresponds to the following deductive rule:

$$
X[M @ \overrightarrow{a r g} s \Rightarrow Y] \leftarrow X:: Z \wedge Z[M @ \overrightarrow{a r g} s \Rightarrow Y]
$$

This rule creates an effect similar to recursive cycles through negation when signatures are negatively dependent on other data. To illustrate the problem Tconsider the following program:

$$
\begin{aligned}
& b:: a \\
& p(a) \leftarrow \neg b[a t t r \Rightarrow c] \\
& a[a t t r \Rightarrow c] \leftarrow p(a)
\end{aligned}
$$

Together with $(30) \Gamma$ we obtain a negative cycle going from $\neg b[a t t r \Rightarrow c]$ to $p(a)$ to $a[a t t r \Rightarrow c]$ to $b[a t t r \Rightarrow c]$.

Fortunately[there is a simple way out of these difficulties (which also works for classical logic programs with equality). The idea is to account for the idiosyncrasies of the equality and of the built-in features of F-logic by adding additional axioms to $\mathbf{P}$ in the form of logical rules. Each of these new axioms Tcalled closure axiomsTcorresponds to an inference rule of Section 10. The only inference rules that do not need axioms are the rules of resolution $\Gamma$ factorization $\Gamma$ merging Tand elimination.

Axioms of paramodulation. For every arity $\Gamma$ $n$ every predicate symbol $\Gamma p$ (including $\doteq$ ) $\Gamma$ and for “ $\sim$ " standing for either of the six arrow types:

$$
\begin{aligned}
p\left(Y_{1}, \ldots, Y_{i}^{\prime}, \ldots, Y_{n}\right) & \longleftarrow p\left(Y_{1}, \ldots, Y_{i}, \ldots, Y_{n}\right) \wedge\left(Y_{i} \dot{\doteq} Y_{i}^{\prime}\right) \\
X\left[M^{\prime} @ A_{1}, \ldots, A_{n} \sim V\right] & \longleftarrow X\left[M @ A_{1}, \ldots, A_{n} \sim V\right] \wedge\left(M \doteq M^{\prime}\right)
\end{aligned}
$$

There are also rules similar to the last one for the paramodulation on $X \Gamma V \Gamma$ and on each of the $A_{i}$. Notice that paramodulation also accounts for the transitivity and the symmetry of the equality predicate. Additionally to account for the paramodulation into subterms $\Gamma$ the following axiom is needed:

$$
\left(f\left(X_{1}, \ldots, X_{i}^{\prime}, \ldots, X_{n}\right) \doteq f\left(X_{1}, \ldots, X_{i}, \ldots, X_{n}\right)\right) \leftarrow\left(X_{i} \doteq X_{i}^{\prime}\right)
$$

for every function symbol $f \in \mathcal{F}$. 
“ $\prec_{\mathbf{P}}$ " and “ $\preceq_{\mathbf{P}}$ " are not partial orders. HoweverTthey are partial orders when $\mathcal{D}_{\mathcal{L}}(\mathbf{P})$ has no cycles that contain negative edges (the negative cycles).

Definition A.1 (Locally Stratified Programs, preliminary) An F-programГP Tis locally stratified if the relation " $\prec_{\mathrm{P}}$ " is well-founded Ti.e. Tthere is no infinite decreasing chain of the form $\cdots \prec_{\mathrm{P}} \phi_{2} \prec_{\mathrm{P}} \phi_{1}$.

This definition implies that for locally stratified programs “ $\prec_{\mathbf{P}}$ " is irreflexive and asymmetric (for $\Gamma$ otherwise $\Gamma$ there would be an infinite chain $\cdots \prec_{\mathbf{P}} \phi_{1} \prec_{\mathbf{P}} \phi_{2} \prec_{\mathbf{P}} \phi_{1} \Gamma$ for some $\phi_{1}$ and $\phi_{2}$ such that $\phi_{1} \prec_{\mathbf{P}} \phi_{2}$ and $\left.\phi_{2} \prec_{\mathbf{P}} \phi_{1}\right)$. SimilarlyCDefinition A.1 implies that the graph $\mathcal{D}_{\mathcal{L}}(\mathbf{P})$ does not have negative cycles when $\mathbf{P}$ is locally stratified.

Next we introduce a preference quasi-order as follows: an H-structure $\mathrm{M}$ is preferable to LTdenoted $\mathbf{M} \ll_{\mathrm{P}} \mathrm{L} \Gamma$ if whenever there is an atom $\varphi$ such that $\varphi \in \mathbf{M}-\mathbf{L}$ and then there is an atom $\psi$ such that $\operatorname{level}_{\mathbf{P}}(\psi)<\operatorname{level}_{\mathbf{P}}(\varphi) \Gamma \psi \in \mathrm{L}-\mathrm{M}$.

Definition A.2 (Perfect Models, preliminary) Let $\mathbf{P}$ be a locally stratified F-program and $\ll_{\mathbf{P}}$ be an associated quasi-order on its H-models. H-models of $\mathbf{P}$ that are minimal with respect to $\ll_{\mathbf{P}}$ are called perfect models of $\mathbf{P}$.

In general $\Gamma \ll_{\mathbf{P}}$ is a quasi-order. However $\Gamma$ as in [81] $\Gamma$ it can be shown that for any locally stratified

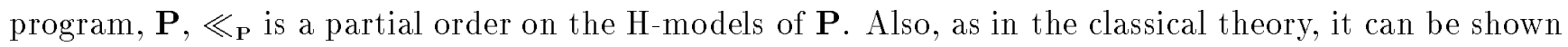
that every locally stratified program has a unique perfect model.

Let $\mathbf{P}$ be a locally stratified program. It is easy to verify that there is a function

$$
\operatorname{level}_{\mathrm{P}}: \mathcal{H B}(\mathcal{F}) \longmapsto \mathcal{N}
$$

where $\mathcal{N}$ is the set of all natural numbers $\Gamma$ such that for any pair $\phi \phi, \psi \in \mathcal{H B}(\mathcal{F}) \Gamma$ if $\phi \preceq_{\mathbf{P}} \psi$ then $\operatorname{level}_{\mathbf{P}}(\phi) \leq \operatorname{level}_{\mathbf{P}}(\psi)$ and if $\phi \prec_{\mathbf{P}} \psi$ then $\operatorname{level}_{\mathbf{P}}(\phi)<\operatorname{level}_{\mathbf{P}}(\psi)$.

Given a level-function $\Gamma$ we can partition the rules in $\mathbf{P}^{*}$ into $\mathbf{P}_{1}^{*}, \mathbf{P}_{2}^{*}, \ldots$ according to the level of the head-literal in each rule. More precisely each $\mathbf{P}_{k}^{*}$ consists of all those rules in $\mathbf{P}^{*}$ whose head-literal is assigned the level $\leq k$. In particular $\Gamma \mathbf{P}_{k}^{*}-\mathbf{P}_{k-1}^{*}$ consists of all rules with head-literals at level $k$.

As in the classical theory it can be shown that the perfect model of $\mathbf{P}$ can be computed by firing logical rules in the stratification order determined by level $_{P} \Gamma$ and that the result does not depend on the choice of the level-function as long as it is synchronized with $\prec_{\mathrm{P}}$ and $\preceq_{\mathrm{P}}$.

Unfortunately the above straightforward adaptation of the notion of perfect models is inadequate. One problem comes from the equality predicate $\Gamma^{\prime \doteq ” . ~ I n ~[81] ~} \Gamma$ perfect models were introduced assuming the so called freeness axioms for equality which postulate that ground terms are equal if and only if they are identical. Without these axiomsT perfect models do not provide natural semantics even for simple locally stratified programs. For instance Cconsider the following pair of F-programs:

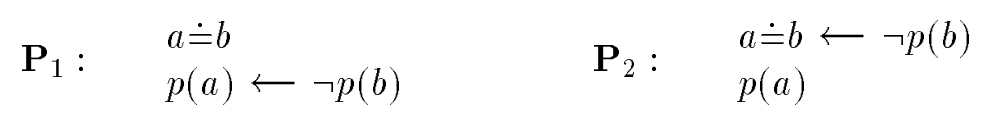


dimension: F-logic is capable of representing almost all aspects of what is known as the object-oriented paradigm. We have provided a formal semantics for the logic and have shown that it naturally embodies the notions of complex objects $\mathrm{inh}$ eritance $\mathrm{methods} \Gamma$ and types. F-logic has a sound and complete resolution-based proof procedure which makes it also computationally attractive and renders it a suitable basis for developing a theory of object-oriented logic programming.

Acknowledgements: We are indebted to Catriel Beeri for the very detailed commentsTalmost as long as this paperTwhich helped us improve the presentation. Many other improvements in this paper are due to the suggestions of Rodney Topor. We are grateful to Alexandre Lefebvre and Heinz Uphoff for pointing out a problem with an earlier version of the semantics of inheritance. We also wish to thank

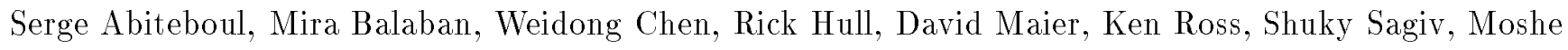
VardiTDavid Warren and Gio Wiederhold for the discussions that helped shape this paper. Last $\mathrm{but}$ not least $\Gamma$ thanks goes to the anonymous referees for their constructive critique.

\section{A Appendix: A Perfect-Model Semantics for F-logic}

The general principle in adapting the various semantics for negation (such as [81Г39Г96Г95Г 82 Г 4 Г 54]) to F-logic is to use method names whenever predicates are used in the standard setting. For instance for the perfect-model semantics [81] stratification must be ensured with respect to method namesTand so the program

$$
X[\text { wants } \rightarrow Y] \leftarrow \neg X[\text { has } \rightarrow Y]
$$

would be considered as stratified despite the recursion through negation within the same object $X$. Note that $\neg x[$ has $\rightarrow y]$ here means that $y \notin x$.has; it does not mean that the value of has on $x$ is empty or undefined. In contrastTconsider the following one-rule program (adapted from [96]):

$$
\begin{aligned}
\text { sillyGame }\left[\text { winningPos } \rightarrow \text { Pos }_{1}\right] \longleftarrow & \neg \text { sillyGame }\left[\text { winningPos } \rightarrow \text { Pos }_{2}\right] \\
& \wedge \text { Pos }_{1}\left[\text { legalMoves } \rightarrow \text { Pos }_{2}\right]
\end{aligned}
$$

It is not considered as locally stratified in F-logic since there is recursion through negation in the method winningPos when both $\operatorname{Pos}_{1}$ and $P o s_{2}$ are instantiated to the same constant Tsay badPos.

HoweverTthe process of adapting perfect models to F-logic is more involved. We present a solution in two steps. FirstTwe apply the above general principle directly and come up with a preliminary definition. Then we point to the inadequacies of this definition and show how problems can be corrected.

Let $\mathcal{L}$ be an $\mathrm{F}$-logic language with $\mathcal{F}$ being its set of function symbols. Consider a general F-program $\Gamma$ $\mathbf{P}$. We define a dependency graph $\Gamma \mathcal{D}_{\mathcal{L}}(\mathbf{P}) \Gamma$ as follows: Let $\mathbf{P}^{*}$ denote the set of all ground instances of $\mathbf{P}$ in $\mathcal{L}$. The nodes of $\mathcal{D}_{\mathcal{L}}(\mathbf{P})$ correspond to the ground atoms in the Herbrand base $\mathcal{H} \mathcal{B}(\mathcal{F})$. A positive $\operatorname{arc} \Gamma \varphi \stackrel{\oplus}{\longleftarrow} \psi$ Connects a pair of nodes $\Gamma \varphi$ and $\psi \Gamma$ if and only if there is a rule $\bar{\varphi} \leftarrow \cdots \wedge \bar{\psi} \wedge \cdots$ Tin $\mathbf{P}^{*}$ such that $\varphi$ and $\psi$ are constituent atoms of $\bar{\varphi}$ and $\bar{\psi}$ Trespectively. A negative $\operatorname{arc} \Gamma \varphi \stackrel{\ominus}{=} \Gamma$ is in $\mathcal{D}_{\mathcal{L}}(\mathbf{P})$ if and only if $\mathbf{P}^{*}$ has a rule $\bar{\varphi} \leftarrow \cdots \wedge \neg \bar{\psi} \wedge \cdots$.

We shall write $\phi \preceq_{\mathbf{P}} \psi$ Twhere $\phi$ and $\psi$ are atoms in $\mathcal{H B}(\mathcal{F}) \Gamma$ if $\mathcal{D}_{\mathcal{L}}(\mathbf{P})$ has a directed path from $\phi$ to $\psi$. We write $\phi \prec_{\mathbf{P}} \psi$ if there is a negative such path (i.e. Tsome arcs on the path are negative). In general $\Gamma$ 
The semantic mapping $\Phi$ can be defined along similar lines. Once the mappings $\Gamma$ and $\Phi$ are defined

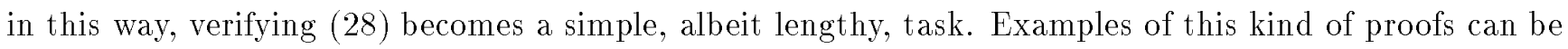
found in [98Г30].

The reader who might be puzzled by the revelation that F-logic isTin a senseTequivalent to predicate

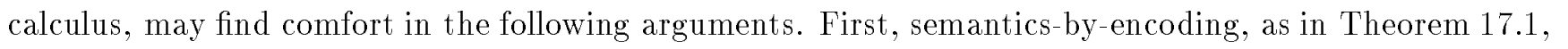
is inadequate as it is indirect and gives little help to the user when it comes to understanding the meaning of a program. Since our declared goal was to provide a logical rendition for a class of languages that are collectively classified as object-oriented $\Gamma$ taking the mapping $\Gamma$ of Theorem 17.1 for the meaning of F-logic would be a misnomer. Indeed $\Gamma \Gamma$ is merely an algorithm that gives little insight into the nature of object-oriented concepts the logic is designed to model 5 and the proof sketch of Theorem 17.1 should make it clear that even for simple F-programs their $\Gamma$-image is not easily understandable. Another argument-well articulated in the concluding section to [30] - can be summarized as follows: The syntax of a programming language is of paramount importance $\mathrm{T}$ as it shapes the way programmers approach and solve problems. Third Ta direct semantics for a logic shows a way of defining a proof theory that is tailored to that logic. Such a proof theory is likely to be a better basis for an implementation than the general-purpose proof theory of the classical logic. LastlyCTheorem 17.1 relates only the monotonic part of F-logic to classical logic. Mapping the non-monotonic components of F-logic into a non-monotonic theory for predicate calculus does not lead to a bonified logic.

\section{Conclusion}

Unlike the relational approach that was based on theoretical grounds from the very beginning Tthe objectoriented approach to databases was dominated by "grass-roots" activity where several implementations existed (e.g. $[53 \Gamma 68]$ ) without the accompanying theoretical progress. As a result $\Gamma$ many researchers had felt that the whole area of object-oriented databases is misguided Tlacking direction and needing a spokesman like Codd $\Gamma$ who could "coerce the researchers in this area into using common set of terms and defining a common goal that they are hoping to achieve [78]."

Our contention is that the problem lies much deeper: when Codd made his influential proposalThe was relying on a large body of knowledge in Mathematical Logic concerning predicate calculus. He had an insight to see a very practical twist to a rather theoretical body of knowledge about mathematical logic which has led him to develop a theory that revolutionized the entire database field. Until now logical foundations for object-oriented databases that are parallel to those underlying the relational theory were lacking and this was a major factor for the uneasy feeling. In his pioneering work [65] TMaier proposed a framework for defining a model-theoretic semantics for a logic with an object-oriented syntax. However $\Gamma$ he encountered many difficulties with his approach and subsequently abandoned this promising direction. As it turned out Tthe difficulties were not fatalГand the theory was repaired and significantly extended in $[31 \Gamma 50]$.

In this paper $\Gamma$ we have proposed a novel logic that takes the works reported in $[31 \Gamma 50]$ to a new 
of canonic models.

Because of the independence of its various componentsГF-logic can be viewed as a tool-kit for designing custom-tailored declarative languages. In fact Tthere is a more fine-grained division inside the logic. For instance $\Gamma$ the semantics of scalar methods is independent from that of set-valued methods $\Gamma$ and we can consider each type of methods as a separate tool. Likewise $\mathrm{T}$ we could require the oid's of classes and methods to be constants; method-name overloading can also be controlled in a number of ways (using sorts $\mathrm{f}$ for example). This tool-kit-like structure of F-logic is extremely helpfulГ as it lets us address different aspects of the logic in separation from each other Tboth theoretically and implementationally.

The last issue we would like to discuss is the relationship between predicate calculus and F-logic. In one direction Tpredicate calculus is a subset of F-logic and so the latter is more powerful than the former. HoweverTit turns out that Tin a sense $\Gamma$ the two logics are equivalent.

Theorem 17.1 There are mappings

$$
\begin{array}{llll}
\Gamma:\{F \text {-formulas }\} & \longmapsto & \{\text { well-formed formulas of predicate calculus }\} \\
\Phi:\{F \text {-structures }\} & \longmapsto & \{\text { semantic structures of predicate calculus }\}
\end{array}
$$

such that

$$
\left.\mathbf{M}\right|_{F} \psi \text { if and only if }\left.\Phi(\mathbf{M})\right|_{P C} \Gamma(\psi)
$$

for any F-structure $\mathrm{M}$ and any $F$-formula $\psi$; here $"={ }_{F}$ " and " $=P C$ " denote logical entailment in F-logic and predicate calculus, respectively.

Proof: (Sketch) The proof is easy but tediousTso most of it is left as an exercise. The main idea is to introduce new predicates that encode the meaning of the methods for every arity and for every type of invocation.

Let scalarNonInheritable $e_{n}$ be the predicate that is chosen to encode $n$-ary non-inheritable scalar data expressions. SimilarlyГ we can choose setNonInheritable ${ }_{n}$ to encode non-inheritable set-valued data expressions. Then we can split F-molecules into constituent atoms and represent each atom by an appropriate tuple in one of the above predicates. In addition $\Gamma$ we shall need predicates scalarType $_{n}$ and setType $_{n}$ to encode scalar and set-valued signature expressions. For instanceI

$$
\text { bob }[j o i n t W o r k s @ p h i l \rightarrow X] \wedge e m p l[\text { budget } \Rightarrow \text { integer }]
$$

can be represented as

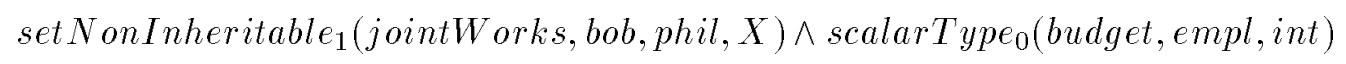

Class membership and subclassing can be represented by appropriate binary predicates. To complete the encoding $\Gamma$ Tit remains to specify axioms that achieve the effect of the built-in features of F-structures. For instance $\Gamma$ it will be necessary to write down a transitivity axiom for subclassing $\Gamma$ type inheritance axiomsTetc. 


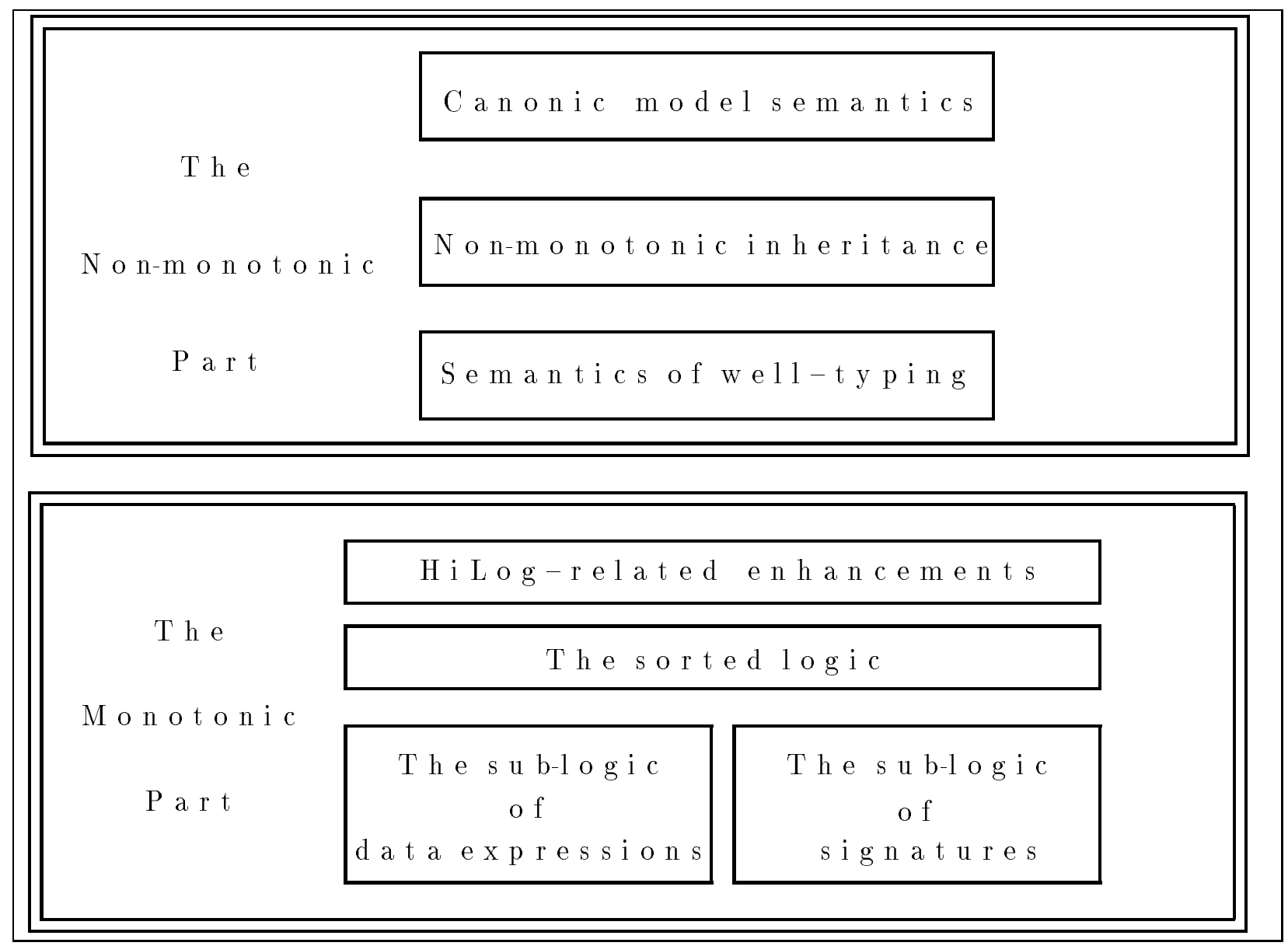

Figure 9: The Internal Structure of F-logic

semantics and the proof theory presented earlier. The glue between these two sublogics is provided by the well-typing semantic conditions of Section 12 Tin the non-monotonic part of the logic. Furthermore $\Gamma$ notice that Tas far as the monotonic logic is concerned Tinheritable and non-inheritable data expressions are completely independent.

The third component is a technique for defining sorts in F-logic. This technique has been discussed in Section 16.1. The fourth component consists of ideas borrowed from HiLog [30] $\Gamma$ which extend the syntax of F-logic to allow variables and even complex terms to appear in the positions of predicate and function symbolsTas discussed in Section 16.2.

The non-monotonic part of F-logic is also composed of a collection of distinct ideas. One of them is the canonic model semantics for F-programs with equalityTas discussed in Section 11 and in Appendix A. The next two determine the semantics of non-monotonic inheritance and typing $\Gamma$ both based on the idea of a canonic model. The notion of encapsulation also belongs here $\mathrm{Tsince}$ we view it simply as an elaborate policy of type-correctness. Note thatTalthough Appendix A defines perfect models as an example of a canonic model semantics Tour treatment of inheritance and typing is independent from this specific theory 
language-engineering issues furtherTas they are beyond the scope of this paper.

\subsection{HiLog-Inspired Extensions}

HiLog [30] is a higher-order extension of predicate calculus that allows variables to range over the names of function and predicate symbols. Furthermore These names themselves may have a rather complex structure and Tin particularTthe usual first-order terms (with variables) may appear in predicate positions. Many applications of HiLog are described in [30Г $100 \Gamma 84]$ and it is clear that extending F-logic in this direction is a good idea. In Section 11.4.1 $\Gamma$ we have seen one example (graph restructuring) where a combination of HiLog and F-logic may be useful.

We illustrate HiLog using the following example:

$$
\begin{aligned}
& \text { closure }(\text { Graph })(\text { From }, \text { To }) \leftarrow \operatorname{Graph}(\text { From }, \text { To }) \\
& \text { closure }(\text { Graph })(\text { From }, \text { To }) \longleftarrow G r a p h(\text { From }, \text { Mid }) \wedge \operatorname{closure}(\text { Graph })(\text { Mid,To })
\end{aligned}
$$

This HiLog program computes the closure of any binary relation that is passed to a predicate constructor $\Gamma$ closure $\mathrm{T}$ as an argument. Here Graph is a variable that ranges over predicates and closure $(G r a p h)$ is a non-ground term that denotes a parameterized family of predicates. For any given binary predicate graph The term closure (graph) is another binary predicate that is true of a pair $\langle$ from,to $\rangle$ precisely when graph has a directed path connecting from and to.

The ideas underlying HiLog are applicable to a wide range of logical languagesTand the reader familiar with HiLog will have no difficulty to integrate them into F-logic by providing a semantics to HiLog-style terms. To this end Tall that has to be done is to change the interpretation of id-terms from the one given in this paper to that in [30].

\section{The Anatomy of F-logic}

It is instructive to take a retrospective look back at the structure of F-logic and clarify the various relationships that exist between its different components. A careful examination would then reveal that most of the components of F-logic are independent from each otherTboth semantically and prooftheoretically. A high-level view of the internals of F-logic is depicted in Figure 9. In the figure $\mathrm{T}$ the logic is presented as a bag of ideas that can be classified into two main categories. The monotonic part of F-logic was presented in Sections 4 through Section 10. The non-monotonic part is comprised of a number of techniques $\Gamma$ all based on the canonic model semantics. These techniques are described in Sections $11 \Gamma 12$ T14 Tand in Appendix A. The non-monotonic part of F-logic is based on the monotonic part Tespecially on its model theory. The proof theory presented in Section 10 is sound and complete for the monotonic part Tbut the non-monotonic extensions cannot have a complete proof theory. This is completely analogous to classical logic where logic programs with stratified negation have no complete proof theory.

The two main components of the monotonic department are the logics of data expressions and the logic of signatures. These sublogics are independent from each otherTas can be seen by inspecting the 


\subsection{Sorted F-logic and its Uses}

In some cases it may be necessary to distinguish between set-valued and scalar attributes at the syntactic

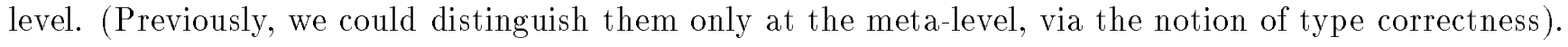

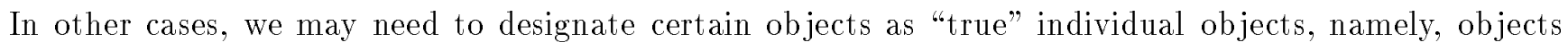
that can never play the role of a class. For instanceГobjects that represent people $\Gamma$ such as john $\Gamma$ mary $\Gamma$ etc. Tmay be in this category. Using sorts to separate things that should not be mingled in the same domain is an old idea. The advantage of having sorts in addition to types is that well-formedness with respect to sorts is easier to verifyTusually much easier.

To illustrate how this idea can be applied to F-logic Tsuppose we wanted to keep a distinction between classes and individuals and yet be able to manipulate both kinds of objects in a uniform way. To this end $\Gamma$ we could separate all variables and function symbols into three categories with different name spaces. For instance Tto represent individuals $\Gamma$ we could use id-terms whose outermost symbol's name begins with a "!"; to represent classes Twe may use id-terms beginning with a "\#"-symbol; and id-terms that begin with any other legal symbol (e.g. Ta letterTas it was so far) can be used to represent any object Tan individual or a class. These latter symbols allow accessing both kinds of objects uniformly without duplicating program clauses to deal with each sort separately.

Semantically sorts are accommodated in a standard way. The domain $\Gamma U$ Tof an F-structure would now consist of two disjoint parts: $U^{\sharp}$ — to interpret class-objects; and $U^{!}$-for individual objects. Variables beginning with a $\sharp$-symbol will then range over the subdomain of classes $\Gamma$ while !-variables will be restricted to the domain of individuals. All other variables will be allowed to range over the entire domain $\Gamma U$. Since we wish to allow class-objects to be constructed out of both $\Gamma$ class-objects and individual-objects $\Gamma$ a function symbol beginning with a "\#" will have the type $U^{n} \mapsto U^{\sharp} \Gamma$ for a suitable $n \Gamma$ while a function symbol beginning with a "!"-sign will have the type $U^{n} \mapsto U^{!}$.

Alternatively the same effect can be achieved by introducing a pair of new unary predicatesTclass $(X)$ and individual $(X)$ Twhere class and individual are true precisely of those ground id-terms whose outermost symbol starts with a “\#” and “!” Trespectively. In addition $\Gamma$ rules and queries should be relativized as follows: For every individual id-term $\Gamma ! T \Gamma$ in the body of a rule $\Gamma$ head $\leftarrow$ body (or in a

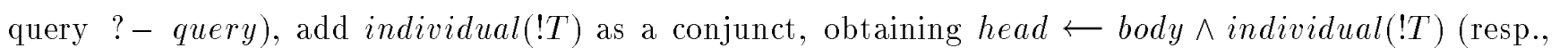
? - query $\wedge$ individual $(! T))$. Likewise $\Gamma$ for every id-term that represents a class $\Gamma \sharp S \Gamma$ add $\operatorname{class}(\sharp S)$ as a conjunct in the body of the rule or the query.

Next we could further restrict logical formulas $\Gamma$ such as signatures and is-a assertions. For instance $\Gamma$ in $T\left[M t h d @ V_{1}, \ldots, V_{n} \Rightarrow W\right] \Gamma$ we may require $T \Gamma V_{1} \Gamma \ldots \Gamma V_{n} \Gamma W$ to represent classes $\Gamma$ (i.e. $\Gamma$ all signatures will actually look like $\sharp T\left[M t h d @ \sharp V_{1}, \ldots, \sharp V_{n} \Rightarrow \sharp W\right]$ ). Likewise $\Gamma$ for is-a assertions of the form $O: C l$ and $C l:: C L^{\prime}$ we may require that $C l$ and $C l^{\prime}$ will be classes.

The above ideas effectively lead to a 3 -sorted logic on top of the basic F-logic. However Tthe utility of sorts is not limited to distinguishing individuals or methods from other objects. In principle $\Gamma$ we may let users define their own naming conventions. In each case $\Gamma$ this will add a new sort of symbols to the logic $\Gamma$ to harbor the oid's of objects the user wishes to distinguish. We shall not discuss these 
equation

$$
\text { current }(\operatorname{chip} 123) \doteq \operatorname{version}(\text { mary, version }(j o h n, v e r \operatorname{sion}(j o h n, \operatorname{chip} 123)))
$$

and delete any other equation of this form that might have been previously asserted.

Navigation through the versions is quite easy. For instanceIto access to the previous version of the chip Tone can use the following query:

$$
?-(\text { current }(\text { chip } 123) \doteq \text { version }(\text { Whoever, PrevVersion })) \wedge \text { PrevVersion }[\ldots] \wedge \ldots
$$

Another way to organize versions in F-logic is to define a method $\Gamma$ version $\Gamma$ that returns appropriate versions $\Gamma$ depending on the argument. To access a version of chip 123 Tone could use

$$
\text { ? - chip } 123[\text { version } @ \arg \rightarrow \text { Version }] \wedge \operatorname{Version}[\ldots]
$$

where $\arg$ is a suitable encoding of the desired version. Yet another way to do versioning is to parameterize attributes with version id's Tas suggested in [45].

\subsection{Path Expressions}

Many calculus-like object-oriented languages (e.g.Г[102Г 14]) use syntactic constructs known as path expressions. For instance $\Gamma$ to select employees working in " $C S$ " departmentsTone could use a path expression X.worksFor.dname $\dot{\doteq}$ "CS" instead of $X[$ worksFor $\rightarrow D[$ dname $\rightarrow$ "CS"]].

The idea of path expressions was significantly extended in [47] and has become a basis of an objectoriented extension of SQL. In the syntax of [47] The expression (ix) of Figure 4 can be written thus: X.worksFor.dname["CS"]. HereГ "CS" plays the role of a selector that selects sequences of objects $x, y, z$ such that $x$. worksFor $=y \Gamma y . d$ name $=z$ Tand $z=$ "CS".

Although path expressions are not explicitly part of F-logic syntax Tthey can be added as a syntactic sugar to help reduce the number variables in F-programs (and also because this notation is more familiar to database users). For instanceГwe could write:

$$
?-X: e m p l \wedge X . n a m e[N] \wedge X . \text { friends.name["Bill"] }] \wedge X . \text { worksFor.dname["CS"] }
$$

instead of the bulkier query:

$$
\text { ?- X:empl[name } \rightarrow N ; \text { friends } \rightarrow F[\text { name } \rightarrow \text { "Bill" }] ; \text { worksFor } \rightarrow D[\text { dname } \rightarrow \text { "CS"] }]
$$

Formal translation of path expressions (as defined in [47]) into F-logic is left to the reader as an easy exercise.

\section{Extensions to F-logic}

In this section Twe explore two enhancements of the logic: the introduction of sorts and extending F-logic in the direction of HiLog [30]. 
where $\mathrm{Tas}$ in Section $6 \Gamma$ p-tuple is a function symbol specificly chosen to represent "invented objects" of class $p$. In other wordsTinvention atoms of [44] are essentially value-based P-molecules of F-logic. ${ }^{26}$

The "*"-style don't-care symbols can be useful for modeling value-based objects $\Gamma$ such as relations with mnemonically meaningful attribute names. For instance Twe could represent the SUPPLIER relation via a class T suppl $\Gamma$ declared as follows:

$$
\text { suppl[name } \Rightarrow \text { string; parts } \Rightarrow \text { part; addr } \Rightarrow \text { string }]
$$

Then we could define the contents of that relation as follows:

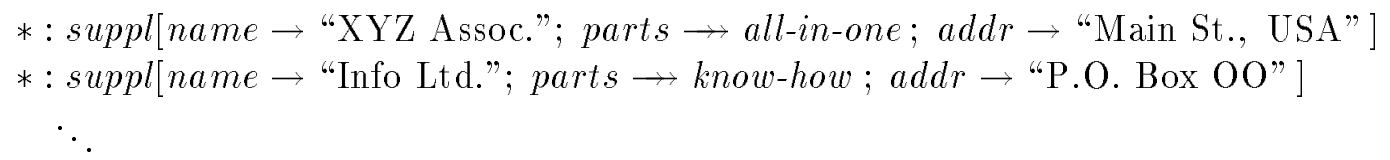

Here $\Gamma$ the symbol "*" is used as a short-hand notation intended to relieve programmers from the tedium of specifying oid's explicitly (which are suppl-tuple("XYZ Assoc.", "Main St. USA") and suppl-tuple("Info Ltd.", "P.O. Box OO") in the above).

It should be clear that the semantics of "*"-style objects is different from the semantics of "_"-style objects. For instance " “*”-style objects are entirely value-based Tas their id's are uniquely determined by their structure. Therefore $\mathrm{an}$ “*”-style object can live its own Tindependent life. Its oid is replaced by "*" simply because this oid is uniquely determined by the values of the scalar attributes of the object $\Gamma$

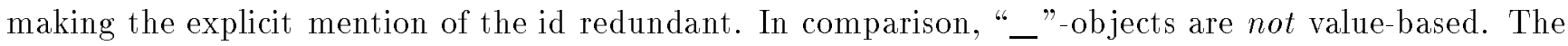
identity of such an object depends mostly on the syntactic position occupied by this object inside another molecule. What the two don't-care styles shareIthough Tis that in both cases the explicit mention of the object identity is redundantTas it is uniquely determined from the context or by the object structure.

\subsection{Version Control}

Although version control is not an intrinsically object-oriented feature $i$ it has been often associated with object-oriented databases because of the importance of versioning in several "classical" object-oriented

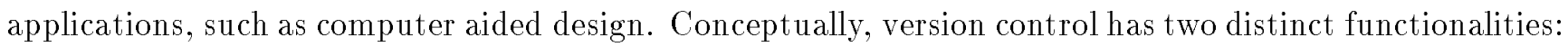
creation of new versions and navigation among the existing versions. As an example $\mathrm{Twe}$ outline one of the several possible schemes for realizing version control in F-logic.

Suppose Mary and John are working cooperatively on a computer chip Tdenoted chip123. Each time Mary makes a modification to a version $\Gamma v \Gamma$ of the chip $\Gamma$ she creates a new object $\Gamma$ version $($ mary, $v)$. SimilarlyГ John constructs versions of the form version $(j o h n, v)$. ThusTall versions of the chip have the form version $\left(p_{n}, \ldots\right.$ version $\left(p_{1}\right.$, chip123) ...) Twhere each $p_{i}$ is either mary or john.

Both Mary and John access the current version of chip123 via the name current(chip123). To turn

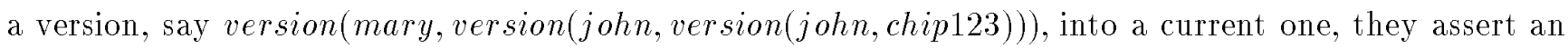

\footnotetext{
${ }^{26}$ However, ILOG is not an entirely value-based language, since there is a way (albeit indirect) to create multiple copies of distinct objects with the same contents.
} 
First Tnote that (i) above is already legal in data expressions. Also (ii) is "almost" legal: it is a generalization of the set-construct where previously we allowed only sets of id-terms on the right-hand side in data expressions.

The next question is the semantics of values introduced in this way. The answer depends on the intended usage of values. If they are taken too literally $\Gamma$ as terms assignable to logical variables $\Gamma$ this could jeopardize the proof theory of our logic. Indeed $\Gamma$ by (i) Tvariables are values and so they can unify with other values. But then the term obj[attr $\rightarrow X]$ should be unifiable with $\Gamma$ say $\Gamma$

$$
o b j[a t t r \rightarrow\{a, b, c\}]
$$

where $X$ unifies with the set $\{a, b, c\}$. In other words $\Gamma$ (i) entails that variables can range over sets $\Gamma$ thereby rendering the semantics second-order in the sense of [30].

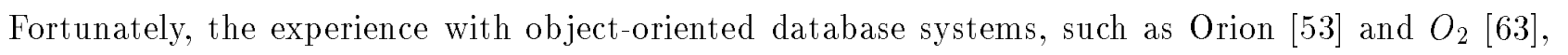
suggests that complex values are used in fairly restricted ways - primarily as weak entities of the EntityRelationship Approach. In other wordsTthey are used as "second-rate objectsT" accessible only through other objects.

This leads to the conclusion that values are used not for their own sake $\mathrm{b} b u t$ rather for convenience $\Gamma$ to let the user omit oid's when they are immaterial. To an extent Tthis is similar to Prolog's don't-care variables represented by underscores. By analogyTwe could permit the use of don't-care object id's. For instance we can re-cast the previous example as follows:

$$
\text { eiffelTower }[\text { name } \rightarrow \text { "Eiffel Tower"; address } \rightarrow \text { _ }[\text { city } \rightarrow \text { paris; street } \rightarrow \text { champDeMars }]]
$$

It remains to decide on a suitable semantics of the don't-care symboll "_". Let

$$
T\left[S \operatorname{cal} M @ S_{1}, \ldots, S_{n} \rightarrow[\ldots]\right]
$$

be a term with a don't-care symbol. It's meaning can be given through the following encoding:

$$
T\left[S \operatorname{cal} M @ S_{1}, \ldots, S_{n} \rightarrow \operatorname{val}_{n}\left(T, S \text { cal } M, S_{1}, \ldots, S_{n}\right)[\ldots]\right]
$$

where $\mathrm{val}_{n}$ is an $(n+2)$-ary new function symbolГspecifically chosen for this kind of encoding. It is not accidental that we have given no interpretation to don't-care symbols inside the set-construct Tas in

$$
T\left[S e t M @ R_{1}, \ldots, R_{m} \rightarrow\{\ldots, \ldots[\ldots], \ldots\}\right]
$$

The meaning and the utility of "_" in this context is unclear to us. However $\Gamma$ below $\Gamma$ we introduce a don"t-care symbol "**" Twith a different semantics $\Gamma$ and this kind of oid may well occur inside sets.

The need for don't-care oid's was also emphasized in ILOG [44] $\Gamma$ where a special "*”-notation was introduced for this purpose. An invention atom $\Gamma p\left(*, t_{1}, \ldots, t_{n}\right) \Gamma$ of ILOG is semantically close to an F-molecule of the form

$$
p \text {-tuple }\left(t_{1}, \ldots, t_{n}\right): p\left[\arg _{1} \rightarrow t_{1} ; \ldots ; \arg _{n} \rightarrow t_{n}\right]
$$


HoweverTwhen the user poses a queryГsuch as

$$
?-j o h n[\text { children } \rightarrow X]
$$

and $X$ gets no bindingsTit is unclear whether this is because John has no children or because this attribute has a null value. To verify this $\Gamma$ the user will have to ask one more query: ? - john[children $\rightarrow\{\}]$.

ClearlyCasking two queries to find out one simple thing is a questionable practice $\mathrm{T}$ and it is highly desirable that a single query $(27)$ Twill suffice. One easy way to fix the problem is to introduce a special

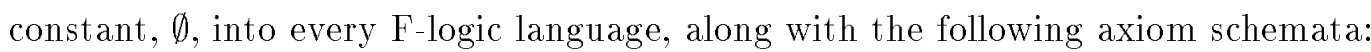

$$
\begin{aligned}
& X\left[M @ A_{1}, \ldots, A_{n} \rightarrow \emptyset\right] \\
& X\left[M @ A_{1}, \ldots, A_{n} \bullet \emptyset\right]
\end{aligned}
$$

where $n=0,1,2, \ldots$. Technically speaking $\Gamma$ these axioms ensure that methods never return empty sets. However $\Gamma$ conceptually $\Gamma e m p t y$ sets can be represented via sets that contain $\emptyset$ alone.

\subsection{Complex Values}

Several recent works [15Г3Г63] have suggested that pure object-based languages may be burdensome when identity of certain objects is immaterial to the programmer. WeTtooThave made this point in Section $6 \mathrm{I}$ advocating the use of predicates on a par with objects. However $\Gamma$ it may sometimes be convenient to go beyond simple predicates and allow object-like structures that have no associated object id. Such structures are called complex values [15Г 3Г 63]. For instanceГadapting an example from [63] we could write:

$$
\text { eiffelTower }[\text { name } \rightarrow \text { "Eiffel Tower"; address } \rightarrow[\text { city } \rightarrow \text { paris; street } \rightarrow \text { champDeMars }]]
$$

Here $\left[\right.$ city $\rightarrow$ paris; street $\rightarrow$ champDeMars] is a "complex value" representing an address. In $O_{2}[63] \Gamma$ such constructs have no identity and are distinct from objects. The rationale is that the user does not have to shoulder the responsibility for assigning id's to objects that are not going to be accessed through these id's.

In the spirit of [3Г63] Twe can try to adapt the concept of complex values to F-logic as follows:

(i) Any id-term is a value;

(ii) $\left\{V a l_{1}, \ldots, V a l_{n}\right\}$ Tis a valueTprovided that each $V a l_{i}$ is a value;

(iii) $[\ldots$, Attr $\sim V a l, \ldots]$ is a value $\Gamma$ where

Attr is an id-term $\Gamma$ Val is a valueTand $\sim$ is one of the four arrows that may occur in data expressions.

To account for complex values T the definition of data expressions in Section 4 should be changed to allow complex values (rather than just oid's) to appear on the right-hand side of data expressions. 
Now $\Gamma$ let $\sup _{1} \Gamma \ldots \Gamma$ sup $_{n}$ denote all the classes where $o b j$ belongs and to which the method mthd applies. Then $o b j$ will inherit the methods $m t h d\left(s u p_{1}\right) \Gamma \ldots \Gamma m t h d\left(s u p_{n}\right)$. This timeThowever $\mathrm{Tthere}$ is no conflict between these methodsTdue to parameterization. So $o b j$ can invoke the method $m t h d$ as defined in any of its superclasses $\Gamma$ e.g. $\Gamma$

$$
?-\operatorname{obj}\left[\operatorname{mthd}\left(\sup _{7}\right) @ a, b \rightarrow Z\right]
$$

This is analogous to the use of scope resolution operators in object-oriented languages.

\section{Other Issues in Data Modeling}

While F-logic has a wide range of object-oriented concepts represented directly in its semantics $\Gamma$ several other ideas have been left out. These include complex values $\Gamma$ path expressions $\Gamma$ and others. In this section Twe propose ways to model these missing concepts using the rich arsenal of the built-in features available in F-logic.

\subsection{Existing and Non-existent Objects}

It is easy to verify that $\mathbf{P}=t[]$ holds for any id-term $\Gamma t \Gamma$ and any program $\Gamma \mathbf{P}$. In this sense $\ln \mathrm{F}$ - $\operatorname{logic} \Gamma$ any object exists as long as it can be named. On the other hand systemsTit is a common practice to distinguish between existing objects and those that do not exist. To come into existenceГeven an empty object must first be created $\Gamma$ which seems to be in stark contrast to F-logic where empty objects[such as $t[]$ above [exist all the time.

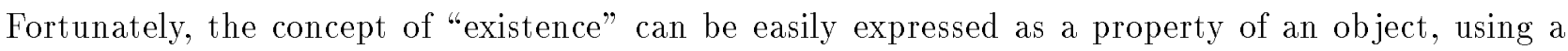

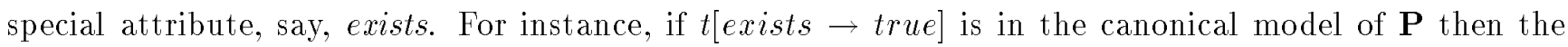
object is said to exist. Otherwise $\mathrm{Tit}$ does not.

Then $\Gamma$ a special preprocessor can modify every clause in $\mathbf{P}$ in such a way that every object molecule that contains a data expression will be augmented with another data expression $\Gamma$ exists $\rightarrow$ true. Queries should be modified accordingly. For instance $T$ the query ?- $X$ will be changed into ?- X[exists $\rightarrow$ true $] \Gamma$ and so only the id's of the existing objects will be retrieved.

\subsection{Empty Sets vs. Undefined Values}

The semantics of set-valued methods distinguishes between the cases when the value of a method is an empty set and when the method is undefined. Although in some cases the user may not care about this distinction Tthis difference is important in many other cases. For instanceTasserting john[children $\rightarrow\{\}]$ means that john has no children (provided that there are no other statements of the form john[children $\rightarrow$ $\rightarrow x y z])$. In contrast $\Gamma$ when $j o h n[$ children $\rightarrow\{\}]$ does not hold Tthis means that it is unknown whether john has children $\Gamma$ as method undefinedness is essentially a form of null values familiar from classical database theory. 
override the default. HoweverTnotice that overriding has taken place only for some specific argumentsT while the default definition is still being used for other arguments (e.g. Twhen Crs $=v l s i)$.

This property of F-logic inheritance is called pointwise inheritance. As we have seen $\Gamma$ pointwise overriding provides a great deal of flexibility by allowing to modify methods over specific subsets of arguments. This should be contrasted with most other languages where methods have to be overwritten in their entirety or not overwritten at all.

\section{Total Method Overriding}

In addition to pointwise overriding $\Gamma i t$ is easy to override methods in their entirety $\mathrm{T}$ as in most objectoriented languages. Consider the following example:

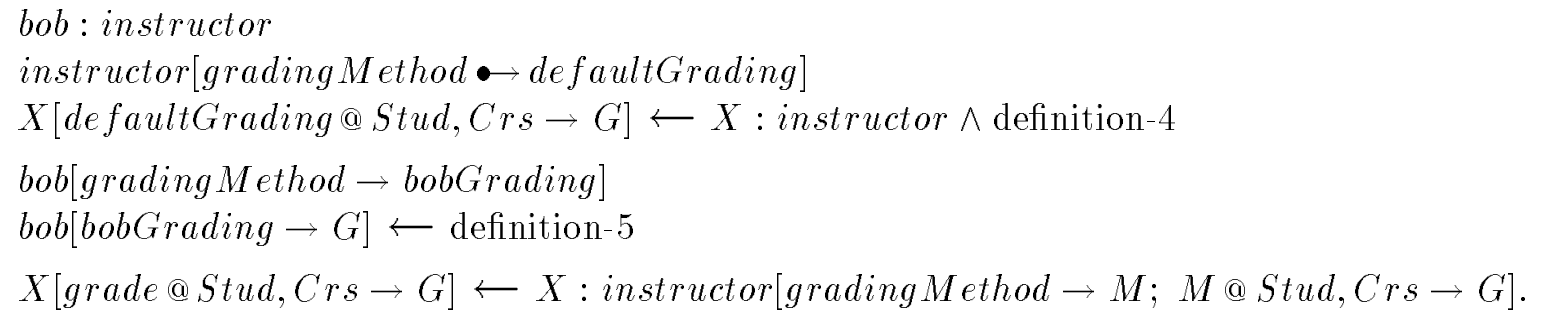

In this exampleTthe value of grading Method is the name of a method to be used for grade computation. This method is defined (in the third clause) for each instructor-object. The fourth clause specifies the name of the grading method that $b o b$ prefers to use. This method is defined on the object $b o b$ using the fifth clause.

The last clause Tthen Tdefines grade using the method $M$ obtained from the grading Method attribute. Normally this value would be inherited from the class instructor and will be defaultGrading. HoweverI the value of the grading Method attribute is overwritten on object bob. Therefore $\mathrm{Twhen} X$ is bound to $b o b \Gamma M$ will be bound to bobGrading and so grade will behave exactly like bobGrading. In contrast $\Gamma$ if mary is also an instructorTbut she did not override the attribute grading Method $\Gamma$ the value for $M$ in the last clause will be inherited from instructor and will thus be defaultGrading. ThereforeTwhen $X$ is bound to mary Tthe method grade will behave exactly like de faultGrading.

\section{User-Controlled Inheritance}

The semantics of inheritance described in this section is non-deterministic i.e. Tin case of an inheritance conflict $\Gamma$ the system will fire one of the active triggers non-deterministically. In some cases $\Gamma$ however $\Gamma$ the user may want to have more control over trigger-firing. This approach is common in many programming languages $\Gamma$ such as Eiffel [70] or $\mathrm{C}++[89] \Gamma$ where the programmer has to resolve inheritance conflicts explicitly.

User-defined inheritance can be expressed in F-logic as follows. To resolve a conflict Tsay method $\Gamma m t h d \Gamma$ we can first parameterize $m$ thd by defining a family of methods $\Gamma m t h d(C l a s s)$ :

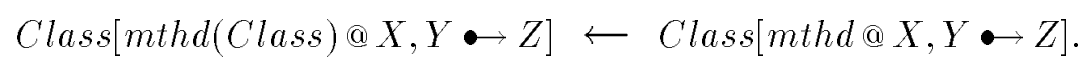




\begin{tabular}{|c|c|c|c|c|}
\hline object & $\mathrm{L}$ & $\mathrm{M}_{1}$ & $\mathbf{M}_{2}$ & $\mathrm{M}_{3}$ \\
\hline$p$ & $a t t r_{1} \bullet c$ & attr $_{1} \bullet c$ & $a t t r_{1} \bullet c$ & $a t t r_{1} \leftrightarrow c$ \\
\hline$t$ & $a t t r_{1} \bullet d$ & $a t t r_{1} \bullet d$ & $a t t r_{1} \bullet d$ & attr $_{1} \bullet d$ \\
\hline$a$ & - & $a t t r_{1} \rightarrow c$ & $a t t r_{1} \rightarrow d$ & $a t t r_{1} \rightarrow c$ \\
\hline$a$ & - & $a t t r_{2} \rightarrow e$ & & attr $_{2} \rightarrow e$ \\
\hline$a$ & - & - & - & attr $_{1} \rightarrow d$ \\
\hline$i s-a$ & $a: p$ & $a: t, t:: p$ & $a: t, t:: p$ & $a: t, t:: p$ \\
\hline
\end{tabular}

Among the above modelsTonly $\mathbf{M}_{1}$ is $\iota$-canonical; it is obtained from $\mathbf{L}$ by firing the previously defined triggers $\tau$ and $\kappa$.

The subtle point here is that if $a: t$ and $t:: p$ were true in the initial model $\mathrm{L} \Gamma$ the existence of $t\left[a_{t t r} \bullet \rightarrow d\right]$ on the path $a: t:: p$ would have blocked the inheritance of attr $1 \bullet \rightarrow c$ from $p$ to $a$. However blocking an inheritance path after the inheritance step is performed does not undo inheritance acts performed earlier.

\subsection{Modeling Other Overriding Strategies}

In this section $\Gamma$ we illustrate various ways in which F-logic inheritance can model overriding strategies used in other languages.

\section{Pointwise Overriding}

Suppose that the class instructor provides a default definition for the method grade used to compute students' grades in different courses:

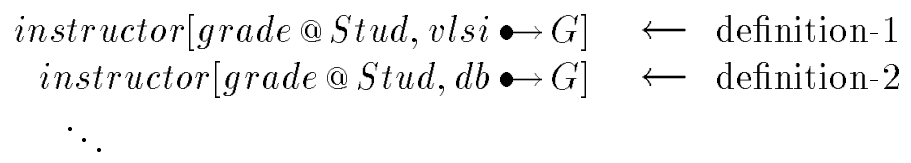

Any instructorTsay $b o b$ (assuming bob : instructor holds) Tcan access this method by asking queries:

$$
?-b o b[\text { grade } @ \text { mary, vlsi } \rightarrow G]
$$

Suppose instructor $[$ grade @ mary,vlsi $\bullet 90]$ holds in class instructor. Then grade@mary, vlsi $\bullet 90$ will be inherited by bobГderiving the atom bob[grade@mary, vlsi $\rightarrow 90]$. Similar inheritance will take place for $d b$ and other courses.

Suppose now that instructors are allowed to modify the default grade computation algorithm by defining their own. ThusTfor instance $b o b$ may define his own policy in the database course:

$$
b o b[\text { grade } @ S t u d, d b \rightarrow G] \leftarrow \text { definition-3 }
$$

This would provide an explicit definition of grade in the object bob when this method is invoked with arguments stud and $d b \Gamma$ where stud represents an arbitrary student. Thus 5 this explicit definition will 


\begin{tabular}{|c|c|c|c|c|}
\hline object & L & $\mathrm{M}_{1}$ & $\mathbf{M}_{2}$ & $\mathrm{M}_{3}$ \\
\hline$q$ & $a t t r \bullet \rightarrow a$ & $a t t r \bullet \rightarrow a$ & $a t t r \bullet \rightarrow a$ & $a t t r \bullet \mapsto a$ \\
\hline$p$ & - & $a t t r \rightarrow a$ & - & $a t t r \rightarrow a$ \\
\hline$r$ & - & $a t t r \rightarrow b$ & $a t t r \longrightarrow a$ & $a t t r \rightarrow a$ \\
\hline$r$ & - & - & - & $a t t r \rightarrow b$ \\
\hline$\overline{i s-a}$ & $p: q, r: q$ & $q, r: q$ & $p: q, r: q$ & $p: q, r: q$ \\
\hline
\end{tabular}

Both $\mathbf{M}_{1}$ and $\mathbf{M}_{3}$ are $\iota$-canonical models. Observe how the rule application that produced $\mathbf{M}_{1}$ from $I_{\mathbf{P}}^{\tau}(\mathrm{L})$ had deactivated the trigger $\Gamma \kappa$. On the other hand $\Gamma$ firing $\kappa$ did not prevent the rule from firing.

The existence of two $\iota$-canonic models for the above program may appear discomforting $\mathrm{Cbecause}$ a case can be made that trigger $\tau$ should have been fired before trigger $\kappa$. Indeed $\Gamma$ firing the former obviates the need to fire the latter and $\Gamma$ in that sense $\Gamma M_{1}$ is a "smaller" model than $\mathbf{M}_{3}$. This can be seen even more clearly if we replace " $\longrightarrow$ " and " $\rightarrow$ " in $(25)$ with " $\rightarrow$ " and " $\bullet$ "Trespectively. In this case model $\mathbf{M}_{3}$ (with double-headed arrows turned into the single-headed ones) would contain the atom $a \doteq b$ and thus also $r[a t t r \rightarrow b]$. This turns $\mathbf{M}_{1}$ into a strict subset of $\mathbf{M}_{3}$ Tfurther fueling our suspicion about the wisdom of keeping $\mathbf{M}_{3}$ as a canonic model.

The problem with premature firing of triggers (as in the case of $\kappa$ above) can be solved by ordering active triggers in certain ways. This issue will be discussed separatelyTin [49].

The following example illustrates the phenomenon of inheritance in dynamically changing IS-A hierarchies. Let the program $Г$ P Гbe:

$$
\begin{array}{ll}
a: p & p\left[a t t r_{1} \bullet c\right] \\
a: t \leftarrow a\left[a t t r_{1} \longrightarrow c\right] & t\left[a t t r_{1} \leftrightarrow d ; a t t r_{2} \bullet e\right] \\
t:: p \longleftarrow a\left[a t t r_{1} \longrightarrow c\right] &
\end{array}
$$

Let $\mathbf{L}$ be the minimum model of $\mathbf{P}$. Then $\tau=\left\langle a t t r_{1}, \bullet, a: p\right\rangle$ is the only active trigger in L. Firing $\tau$ causes $a: t$ and $t:: p$ to be derived. This $\Gamma$ in turn $\Gamma$ activates the trigger $\kappa=\left\langle\right.$ attr $\left._{2}, \bullet, a: t\right\rangle \Gamma$ and $a\left[a t t r_{2} \rightarrow e\right]$ is derived by inheritance. Note that there is no inheritance of $a t t r_{1} \bullet d$ from $t$ to $a \Gamma$ since $a^{t} t_{1}$ is already defined on $a$.

The most interesting aspect of this example is that the very act of inheritance alters the IS-A hierarchy on which it depends. MoreoverTthe derivation of $a: t$ and $t:: p$ propels the fact $t\left[a t t r_{1} \bullet d\right]$ with an inheritable property right in the middle of the path through which $a$ had inherited $a t t r_{1} \bullet c$ from $p$. This issue was already discussed in connection with Example (24) earlierTwhere we had argued that the above inheritance step should not be undoneTdespite the changes in the IS-A hierarchy.

To see how this dynamic situation is taken care of by our semanticsTconsider the following table that presents the relevant parts of several models of interest: 
We thus see that both $\Gamma M_{2}$ and $\mathbf{M}_{3}$ Tare obtained from $\mathbf{M}_{1}$ Talbeit by firing different triggers. These triggers are associated with the same attributeГ policy $\Gamma$ and the same recipient object $\Gamma$ nixon $\Gamma$ but with different contributing superclassesTquaker and republican. For this reasonTinheritance steps obtained by firing each trigger separately cannot be combined and vice versa. The existence of a pair of triggers with these properties is a formal explanation for the phenomenon of multiple inheritance.

The following result is a direct consequence of the definitions:

Lemma 14.4 Let $\mathbf{P}$ be a Horn F-program and let $\mathbf{I}$ be an H-structure for $\mathbf{P}$. Let, further, $\tau$ be an active trigger in $\mathbf{I}$. Then $T I_{\mathbf{P}}^{\tau}(\mathbf{I})=\left(T_{\mathbf{P}} \uparrow \omega\right)\left(I_{\mathbf{P}}^{\tau}(\mathbf{I})\right)$, where $T_{\mathbf{P}} \uparrow \omega$ denotes the countably-infinite iteration of $T_{\mathbf{P}}$.

This lemma leads to a result Tbelow Tthat sheds light on the computational properties of $\iota$-canonical models of Horn programs. First $\Gamma$ we need to define iterations of the operator $T I_{\mathbf{P}}$. Since $T I_{\mathbf{P}}^{\tau}$ is monotone with respect to $\mathrm{H}$-structures that have $\tau$ as an active trigger $\Gamma$ iterations can be defined in a standard way where we only have to take care of the non-deterministic selection of triggers.

- If $\alpha=0$ Tthen $\left(T I_{\mathbf{P}}^{\tau} \uparrow \alpha\right)(\mathbf{I})=$ ITfor any trigger $\tau$.

- If $\alpha$ is a non-limit ordinal $\Gamma\left(T I_{\mathbf{P}} \uparrow \alpha\right)(\mathbf{I})=T I_{\mathbf{P}}^{\tau}\left[\left(T I_{\mathbf{P}} \uparrow(\alpha-1)\right)(\mathbf{I})\right] \Gamma$ for any trigger $\tau$ that is active in $\left(T I_{\mathbf{P}} \uparrow(\alpha-1)\right)(\mathbf{I})$. That is $\Gamma$ the result of the transition from $\left(T I_{\mathbf{P}} \uparrow(\alpha-1)\right)(\mathbf{I})$ to $\left(T I_{\mathbf{P}} \uparrow \alpha\right)(\mathbf{I})$ is non-deterministic $\Gamma$ as it depends on the trigger selected.

- If $\alpha$ is a limit ordinal $\Gamma\left(T I_{\mathbf{P}} \uparrow \alpha\right)(\mathbf{I})=\bigcup_{\beta<\alpha}\left(T I_{\mathbf{P}} \uparrow \beta\right)(\mathbf{I})$.

Proposition 14.5 Let $\mathbf{P}$ be a Horn F-program and $\mathrm{L}$ be its minimum model. Then $\mathbf{P}$ has at least one ı-canonical model, which can be obtained by iterating $T I_{\mathbf{P}}$ using an appropriate sequence of triggers and taking $\mathbf{L}$ as the initial H-structure. Conversely, $\left(T I_{\mathbf{P}} \uparrow \omega\right)(\mathbf{L})$ is an $\iota$-canonical model of $\mathbf{P}$, where $\omega$ is the first infinite ordinal.

The next F-program $Г$ P $\Gamma$ illustrates one of the many ways in which inheritance can interact with deduction:

$$
\begin{array}{ll}
p: q & q[a t t r \bullet a] \\
r: q & r[a t t r \longrightarrow b] \leftarrow p[a t t r \longrightarrow a] .
\end{array}
$$

There are two triggers $\Gamma \tau=\langle$ attr $, \bullet, p: q\rangle$ Tand $\kappa=\langle$ attr $, \bullet, r: q\rangle$. Observe that applying $\tau$ will activate the last clause. Let $\mathbf{L}$ be the minimum model of $\mathbf{P}$ Tand let $\mathbf{M}_{1}, \mathbf{M}_{2}$ be the two models that emerge after firing one of the triggers in $\mathbf{L}$ i.e. $\Gamma T I_{\mathbf{P}}^{\tau}(\mathbf{L})=\mathrm{M}_{1}$ and $T I_{\mathbf{P}}^{\kappa}(\mathbf{L})=\mathbf{M}_{2}$. It is easy to verify that applying the first trigger $\Gamma \tau$ Tdeactivates $\kappa$ Tbut firing $\kappa$ does not deactivate $\tau$. Therefore $\Gamma$ there is no trigger to fire in $\mathbf{M}_{1}$ and no further inheritance is possible. However $\tau$ can still be fired in $\mathbf{M}_{2}$ Cleading to another model $\Gamma \mathrm{M}_{3}=T I_{\mathbf{P}}^{\tau}\left(\mathrm{M}_{2}\right)$. The following table shows the relevant parts of these models: 
Note that $T I_{\mathbf{P}}^{\tau}$ is monotonic with respect to H-structures that have $\tau$ as a trigger $\Gamma$ since $I_{\mathbf{P}}^{\tau}$ is also monotonic on such structures.

We are now ready to define the notion of canonical models that take inheritance into account.

Definition 14.3 (Canonical Models with Inheritance) Let $\mathbf{P}$ be a Horn F-program. A model $\mathbf{M}$ of $\mathbf{P}$ is inheritance-canonical (or $\iota$-canonical) if and only if $\mathrm{M}$ is a minimal element with respect to $\ll^{\text {inh }}$ in the set $\left\{\mathbf{N} \mid \mathbf{N} \ll^{i n h} \mathbf{L}\right\}$ of $\mathrm{H}$-models $\Gamma$ where $\mathbf{L}$ is the unique minimum model of $\mathbf{P}$ (which exists $\mathrm{s}$ since $\mathbf{P}$ is Horn (by assumption).

We shall now illustrate the notions of one-step inheritance and of $\iota$-canonical model on a number of examples. The following two examples are derived from the Nixon's Diamond example.

Consider the following program $\Gamma \mathbf{P}$ :

$$
\text { nixon }: \text { republican } \quad \text { republican }[\text { policy } \bullet \text { hawk }]
$$

This states that nixon belongs to the class republican and that TgenerallyTrepublicans are hawks. $\mathbf{P}$ has the following models of interest that are summarized below:

\begin{tabular}{l|cl} 
& $\mathrm{M}_{1}$ & \multicolumn{1}{c}{$\mathrm{M}_{2}$} \\
object & & policy $\bullet$ hawk \\
\hline republican & policy $\bullet$ hawk & policy $\rightarrow$ hawk \\
nixon & - & nixon $:$ republican
\end{tabular}

Here $\mathbf{M}_{1}$ is a minimal model of $\mathbf{P}$ Tbut it is not $\iota$-canonical. To see this $\Gamma$ note that $\mathbf{M}_{2}=I_{\mathbf{P}}^{\tau}\left(\mathbf{M}_{1}\right) \Gamma$ where $\tau=\langle$ policy, $\bullet$, nixon $:$ republican $\rangle$ Tand that Tin fact $\Gamma \mathrm{M}_{2}$ is derived from $\mathbf{M}_{1}$ by one inheritance step. It is easy to verify that $\Gamma$ furthermore $\Gamma \mathrm{M}_{2}$ is an $\iota$-canonical $\mathrm{H}$-model of the program. If $\Gamma$ however $\Gamma \mathbf{P}$ contained a clause nixon[policy $\rightarrow$ pacifist] $\Gamma$ then no inheritance could have taken place $\mathrm{Cbecause}$ the more specific valueTpacifist Toverrides the inheritance of the value hawk from the class republican.

The next example illustrates multiple inheritance. Let the program $\mathbf{P}$ be:

$$
\begin{array}{ll}
\text { nixon }: \text { republican } & \text { republican }[\text { policy } \bullet \text { hawk }] \\
\text { nixon }: \text { quaker } & \text { quaker }[\text { policy } \bullet \text { pacifist }]
\end{array}
$$

The following H-models are of interest here:

\begin{tabular}{l|lll} 
& $\mathbf{M}_{1}$ & \multicolumn{1}{c}{$\mathbf{M}_{2}$} & \multicolumn{1}{c}{$\mathbf{M}_{3}$} \\
object & & & \\
\hline republican & policy $\bullet$ hawk & policy $\bullet$ hawk & policy $\bullet$ hawk \\
quaker & policy $\bullet$ pacifist & policy $\bullet$ pacifist & policy $\bullet$ pacifist \\
nixon & - & policy $\rightarrow$ hawk & policy $\rightarrow$ pacifist \\
\hline is-a & nixon $:$ republican & nixon $:$ republican & nixon $:$ republican \\
& nixon $:$ quaker & nixon $:$ quaker & nixon $:$ quaker
\end{tabular}

These models are related as follows: $I_{\mathbf{P}}^{\tau}\left(\mathrm{M}_{1}\right)=\mathrm{M}_{2}$ and $I_{\mathbf{P}}^{\kappa}\left(\mathrm{M}_{1}\right)=\mathbf{M}_{3}$ Twhere $\tau=\langle$ policy, $\bullet$, nixon: republican $\rangle$ and $\kappa=\langle$ policy, $\rightarrow$, nixon : quaker $\rangle$. Furthermore $i$ it is easy to check that $\mathbf{M}_{2}$ and $\mathbf{M}_{3}$ are both $\iota$-canonical models of $\mathbf{P}$ Twhile the model $\mathbf{M}_{1}$ is minimal but not $\iota$-canonical. 
The intention of firing a trigger of the form $\tau=\left\langle m, \bullet, o b j: c l, a_{1}, \ldots, a_{n}\right\rangle \Gamma$ is to inherit to the recipient object $\Gamma$ obj $\Gamma$ the result of the application of $m$ on the source-class. To capture this idea $\Gamma$ we introduce an operator of firing an active trigger $\Gamma$ Ton an $\mathrm{H}$-structure $\mathrm{I}$. This operator is denoted $I_{\mathbf{P}}^{\tau}$ and is defined as follows: ${ }^{25}$

$$
I_{\mathbf{P}}^{\tau}(\mathbf{I})=\mathbf{I} \cup\left\{o b j\left[m @ a_{1}, \ldots, a_{n} \rightarrow v\right] \mid v \in \mathbf{I}\left(c l\left[m @ a_{1}, \ldots, a_{n}\right], \bullet\right)\right\}
$$

Note that since $o b j$ is a member of $c l$ the inheritable properties of $c l$ are passed down to $o b j$ as noninheritable properties. On the other hand $\Gamma$ if $o b j$ were a subclass of $c l$ i.e. Tif the trigger had the form $\kappa=\left\langle m, \bullet, o b j:: c l, a_{1}, \ldots, a_{n}\right\rangle \Gamma$ then the properties of $c l$ would have been passed down to $o b j$ as inheritable properties:

$$
I_{\mathbf{P}}^{\kappa}(\mathbf{I})=\mathbf{I} \cup\left\{\operatorname{obj}\left[m @ a_{1}, \ldots, a_{n} \bullet v\right] \mid v \in \mathbf{I}\left(c l\left[m @ a_{1}, \ldots, a_{n}\right], \bullet\right)\right\},
$$

For scalar invocations $\Gamma$ this operator is defined similarly by replacing $\rightarrow$ with $\rightarrow$ and $\bullet \rightarrow$ with $\bullet$.

Note that $I_{\mathbf{P}}^{\tau}$ is a monotonic operator on the H-structures that have $\tau$ as an active trigger. Once a trigger is fired $\Gamma$ program rules that were previously inactive may become applicable and more facts can be deduced. For instance $\Gamma$ consider the following program $\mathbf{P}$ :

$$
\begin{aligned}
& a: b \\
& b[a t t r \bullet c] \\
& a[a t t r \longrightarrow d] \longleftarrow a[\text { attr } \longrightarrow c]
\end{aligned}
$$

The minimal model here consists of $a: b$ and $b[a t t r \rightarrow c]$. Firing the trigger $\langle a t t r, \bullet, a: b\rangle$ introduces a new fact $\Gamma a[a t t r \rightarrow c]$ Tand the resulting H-structure is no longer a model $\Gamma$ as it violates the last clause. Therefore Tto obtain a model that accommodates the inherited fact Twe have to apply the last rule Twhich derives $a[$ attr $\rightarrow d]$. Note that derivations that are performed after trigger-firing may affect both the recipient object of the trigger (as shown above) and the source-class (as was shown earlier Tin (23)).

The above ideas can be captured via the notion of one-step inheritance Tdefined below. In this paper we limit our attention to the case of Horn F-programs. An extension of this semantics to include programs with negation is reported in [49].

Definition 14.2 (One Step Inheritance Transformation) Let $\mathbf{P}$ be a Horn F-program $\mathbf{I}$ and $\mathbf{J}$ be Hmodels of $\mathbf{P}$ Tand $\tau$ be a trigger in $\mathbf{I}$. We say that $\mathbf{J}$ is obtained from $\mathbf{I}$ via $\tau$ by one step of inheritance $\Gamma$ written $T I_{\mathbf{P}}^{\tau}(\mathbf{I})=\mathbf{J}$ Tif and only if $\mathbf{J}$ is the unique minimal model among those models of $\mathbf{P}$ that contain $I_{\mathbf{P}}^{\tau}(\mathbf{I})$.

When the actual trigger $\Gamma \tau$ Tis immaterial $\Gamma$ we shall write $\mathbf{J} \ll_{1}^{i n h}$ IГand $\ll^{i n h}$ will denote the reflexive and transitive closure of $\ll_{1}^{i n h}$. Informally $\mathbf{J} \ll^{i n h} \mathbf{I}$ means that when inheritance is taken into account $\Gamma$ $\mathbf{J}$ is preferable to $\mathbf{I}$ because it has more inherited facts.

\footnotetext{
${ }^{25}$ Since $\mathbf{I}\left(c l\left[m @ a_{1}, \ldots, a_{n}\right], \rightarrow\right)$ may contain $\emptyset, v$ may denote this element. In this case, obj $\left[m @ a_{1}, \ldots, a_{n} \rightarrow v\right]$ should be understood as obj $\left[m @ a_{1}, \ldots, a_{n} \rightarrow\{\}\right]$.
} 
$\bullet \rightarrow$ ) as follows:

$$
\begin{aligned}
\mathrm{I}\left(p\left[m @ a_{1}, \ldots, a_{n}\right], \rightarrow\right)= & \left\{v \mid p\left[m @ a_{1}, \ldots, a_{n} \rightarrow v\right] \in \mathrm{I}, v \in U(\mathcal{F})\right\} \\
\mathrm{I}\left(p\left[m @ a_{1}, \ldots, a_{n}\right], \bullet\right)= & \left\{v \mid p\left[m @ a_{1}, \ldots, a_{n} \bullet v\right] \in \mathrm{I}, v \in U(\mathcal{F})\right\} \\
\mathrm{I}\left(p\left[m @ a_{1}, \ldots, a_{n}\right], \rightarrow\right)= & \left\{v \mid p\left[m @ a_{1}, \ldots, a_{n} \rightarrow v\right] \in \mathrm{I}, v \in U(\mathcal{F})\right\} \\
& \cup\left\{\emptyset \mid \text { if } p\left[m @ a_{1}, \ldots, a_{n} \rightarrow\{\}\right] \in \mathrm{I}\right\} \\
\mathrm{I}\left(p\left[m @ a_{1}, \ldots, a_{n}\right], \bullet\right)= & \left\{v \mid p\left[m @ a_{1}, \ldots, a_{n} \bullet v\right] \in \mathrm{I}, v \in U(\mathcal{F})\right\} \\
& \cup\left\{\emptyset \mid \text { if } p\left[m @ a_{1}, \ldots, a_{n} \bullet\{\}\right] \in \mathrm{I}\right\}
\end{aligned}
$$

Observe that $\mathbf{I}\left(p\left[m @ a_{1}, \ldots, a_{n}\right], \rightarrow\right)$ is either emptyTwhen $m$ is undefined on $p$ as a set-valued method (invoked with the arguments $\left.a_{1}, \ldots, a_{n}\right)$; or it is a heterogeneous set that contains $\emptyset$ (the empty set) as an ele-

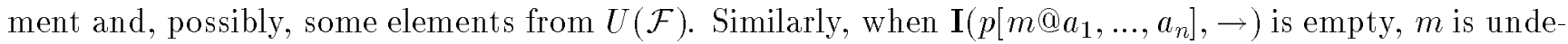
fined on $p$ Twhen it is interpreted as a scalar method. Otherwise $\Gamma i f\left(m\right.$ is defined $\Gamma$ then $\mathbf{I}\left(p\left[m @ a_{1}, \ldots, a_{n}\right], \rightarrow\right)$ is a homogeneous set of elements taken from the Herbrand universe $U(\mathcal{F})$. Note that this set may contain more than one element (even though the invocation is scalar) because of the effects of the equality operator: if $t \in \mathrm{I}\left(p\left[m @ a_{1}, \ldots, a_{n}\right], \rightarrow\right)$ and $(t \doteq s) \in \mathrm{I} \Gamma$ then also $s \in \mathrm{I}\left(p\left[m @ a_{1}, \ldots, a_{n}\right], \rightarrow\right)$. Similar observations apply to $\mathbf{I}\left(p\left[m @ a_{1}, \ldots, a_{n}\right], \bullet\right)$ and $\mathbf{I}\left(p\left[m @ a_{1}, \ldots, a_{n}\right], \bullet\right)$.

Definition 14.1 (Inheritance Triggers) Let I be an H-structure. Consider a tuple $\tau=\langle m, \bullet$, $\left.o b j \sharp c l, a_{1}, \ldots, a_{n}\right\rangle \Gamma$ where $o b j, c l, m, a_{1}, \ldots, a_{n} \in U(\mathcal{F})$ are ground id-terms $\Gamma$ and $\sharp$ denotes the type of the is-a relation (":" or "::") that exists between $o b j$ and $c l$. We shall say that $\tau$ is an active inheritance trigger in I (orTjust a trigger Tfor brevity) if and only if the following conditions hold:

- $o b j \sharp c l \in \mathrm{II}^{24}$ and there is no intervening class $\Gamma$ mid $\in U(\mathcal{F}) \Gamma$ such that mid $\neq c l$ and $o b j \sharp$ mid, mid :: $c l \in \mathrm{I}$;

- The method $m$ is defined in $\mathbf{I}$ as an inheritable property of $c l$ with arguments $a_{1}, \ldots, a_{n} ;$ i.e. $\Gamma \mathrm{I}\left(c l\left[m @ a_{1}, \ldots, a_{n}\right], \bullet\right) \neq \emptyset ;$ and

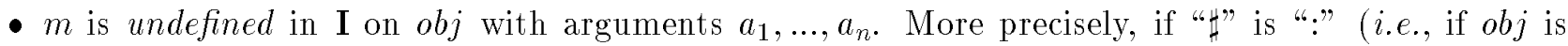
a member of $c l)$ then it must be the case that $\mathbf{I}\left(o b j\left[m @ a_{1}, \ldots, a_{n}\right], \rightarrow\right)=\emptyset$. Otherwise $\mathrm{Cif}$ "\#" is a subclass relationship $\Gamma::$ " Tthen $\mathbf{I}\left(\operatorname{obj}\left[m @ a_{1}, \ldots, a_{n}\right], \bullet\right)=\emptyset$.

Triggers are defined for set-valued invocations of methods similarly by replacing $\rightarrow$ with $\rightarrow$ and $\bullet$ with $\bullet \rightarrow$.

It is clear from the definition that an inheritance trigger is like a loaded gun waiting to fire. Firing a trigger leads to a derivation by inheritance of new facts that cannot be derived using classical deduction. After firing Tthe trigger is "deactivated" andTin factTis no longer a trigger in the resulting H-structure.

Given a trigger of the above form Twe will say that $o b j$ is a recipient object of the impending inheritance step and $c l$ is the source class. Note that $o b j$ here may represent an individual object as well as a subclass.

\footnotetext{
${ }^{24}$ That is, either $o b j: c l$ or $o b j:: c l$ is in I, depending on the specific relationship denoted by "\#”.
} 
Our choice is to not undo inheritance in such a case and Tat the same time to not inherit the newly derived fact $\Gamma b[a t t r \bullet d]$. The reason for the former is primarily computationalTas this leads to much less backtracking. The reason for the latter is aesthetic Tas it leads to a more uniform definition. Another way of looking at this is as follows: once the initial inheritance has taken placeCthe attribute attr is already defined on $a$ and so by the time $b[$ attr $\leftrightarrow d]$ is derived the inheritance of attr $\leftrightarrow d$ by $a$ is overwritten.

To illustrate the problems that (iii) may causeTconsider the following program:

$$
\begin{array}{ll}
a: p & p[a t t r \bullet c] \\
a: t \leftarrow a[\text { attr } \rightarrow c] & t[\text { attr } \bullet d] \\
t:: p \longleftarrow a[\text { attr } \rightarrow c] &
\end{array}
$$

In this example $\Gamma$ if $a$ inherits $a t t r \rightarrow c$ from $p$ the two deductive rules in the program are activated and then both $\Gamma a: t$ and $t:: p \Gamma$ are derived. In other words $\Gamma t$ is propelled into the middle of the inheritance path from $p$ to $a$. What is unusual here is the fact that the attribute attr is already defined on $t$ and has a value $\Gamma d \Gamma$ which is different from $c$. This means that Thad $t$ existed on the inheritance path right from the startTthe inheritance of $\operatorname{attr} \bullet c$ from $p$ to $a$ would have been written overTand $a$ would have inherited attr $\bullet d$ instead. However $\Gamma i n(24) \Gamma t$ was not on the inheritance path initially and so the above argument does not apply. NeverthelessTone can argue that the subsequent derivation of $a: t$ and $t:: p$

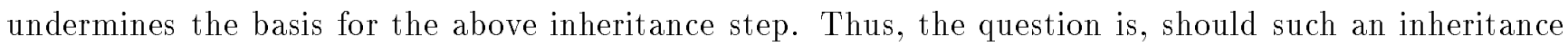
step be undone?

Again Tour choice is to not undo such steps. One important reason is $\Gamma$ as before $\Gamma$ the efficiency of the computation. The other-no less important-reason is that this leads to a very simple semantics whose implications are easy to follow.

In a nutshell the idea is to decompose each inheritance step into a pair of sub-steps: 1) a "pure" inheritance step Twhich may introduce new facts but whose output H-structure may no longer be a model of the original program; and 2) a derivation step that turns the result of the previous step into a model. These operations are repeated until inheritance can be done no more.

In this connection Twe should mention the recent work [34] which also proposes a semantics for inheritance in a language derived from F-logic. In particular Tthis work addresses the issue of the interaction between inheritance and deduction. HoweverTit does not account for set-valued methods and for dynamically changing IS-A hierarchies $\Gamma$ which are two of the three difficult issues listed above. This approach is also fundamentally different from ours in the way semantics is defined. What [34] calls a "canonic model" of a program is actually not a model of that program in the usual sense. Instead Trules that are found to be written over do not have to be satisfied. This aspect of the semantics in [34] is analogous to the work on ordered logic [61Г62] $\Gamma$ discussed earlier. In contrast Tour semantics is more traditional and $\Gamma$ in particularTour "canonic models" are also models in the usual sense.

\subsubsection{A Fixpoint Semantics for Non-monotonic Inheritance}

Let $\mathbf{I}$ be an $\mathrm{H}$-structure and $p, m, a_{1}, \ldots, a_{n}$ be ground id-terms. We define the result of application of a method $\Gamma m$ Ton the object $p$ with arguments $a_{1}, \ldots, a_{n}$ and for a given invocation type $(\rightarrow \Gamma \rightarrow \Gamma \bullet \Gamma$ or 
each canonical model as a viable "possible world" and so any one of these models can be chosen nondeterministically; the other interpretation is that only the facts that belong to the intersection of all such models can be trusted. Since our primary concern is programmingTwe adopt the former view. In other words when inheritance conflict occurs due to multiple inheritance any one of the canonic models can be selected non-deterministically and then used to answer queries.

In a different setting $\Gamma$ non-monotonic inheritance was also discussed in [61Г $62 \Gamma 21]$. According to these approaches $\Gamma$ a logical object (or a class) is a set of rules that represent "knowledge" embodied by the object (resp.Tclass). These rules can be inherited by objects that are placed lower in the hierarchy. Inheritance can be overwritten based on various criteria (e.g. T the existence of overriding rules $\Gamma$ as in [61Г62] Tor because of higher-priority rules Tas in [21]).

Apart from the very different setup in which behavioral inheritance takes place in these works $\mathrm{there}$ is another fundamental difference with our approach (and $\Gamma$ for that matter $\Gamma$ with [36Г 91] and related works). NamelyTin [61Г62Г21] Twhat is inherited is a set of program clauses Twhile in F-logic it is ground data expressions that are passed down the IS-A hierarchy. The latter approach seems to be more flexible. As shown in Section 14.3ГF-logic can easily account for inheritance of clauses and for some other forms of inheritance (see Section 14.3) Twhose representation in the framework of [61Г62Г21] is not obvious to 115.

\subsubsection{Informal Introduction to the Approach}

To integrate inheritance into an object-oriented logic programming system such as F-logic $\Gamma$ the following issues must be addressed:

(i) The interaction between inheritance and ordinary logical deduction;

(ii) Inheritance of sets; and

(iii) The dynamic nature of the IS-A hierarchy.

These issues appear to be closely related andTin fact it is (ii) and (iii) that makes (i) a hard problem. The difficulty is that after inheritance is done $\mathrm{T}$ a program clause may become "activel" causing other facts to be derived. This new inheritance may affect the recipient object and Teven more curiously source-class of inheritance. To illustrate consider the following program:

$$
\begin{aligned}
& a: b \\
& b[a t t r \leftrightarrow c] \\
& b[a t t r \bullet d] \longleftarrow a[\text { attr } \longrightarrow c]
\end{aligned}
$$

If $a$ inherits attr $\leftrightarrow c$ from $b$ The atom $a[$ attr $\rightarrow c]$ is derived and the last clause is activated. This causes the derivation of $b[a t t r \bullet d]$. The question now is $\Gamma$ should the inheritance be "undone" in such a case (because - it can be reasoned - the object $a$ should have inherited attr $\bullet\{c, d\}$ in its entirety or nothing at all)? It seems that different decisions are possible hereTand in some cases the choice may be the matter of taste. 
monly known as Nixon's Diamond:

$$
\begin{array}{ll}
\text { nixon }: \text { quaker } & \text { quaker }[\text { policy } \bullet \text { pacifist }] \\
\text { nixon }: \text { republican } & \text { republican }[\text { policy } \bullet \text { hawk }]
\end{array}
$$

As a member of two classes $\Gamma$ quaker and republican $\Gamma$ nixon can inherit either policy $\rightarrow$ pacifist or

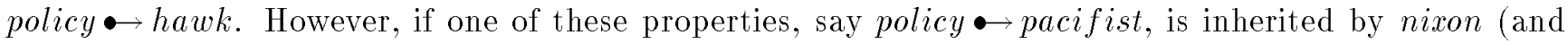
becomes a non-inheritable propertyГpolicy $\rightarrow$ pacifist $)$ Tthen the other property can no longer be inherited because $\mathrm{in}$ the new state $\mathrm{T}$ the attribute policy is defined on the object nixon $\Gamma$ and this definition

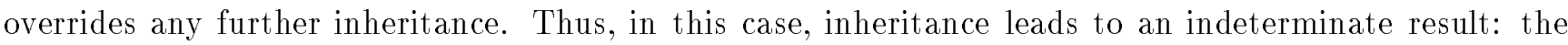
derivation of nixon[policy $\rightarrow$ pacifist $]$ or nixon[policy $\rightarrow$ pacifist $]$ [depending on which inheritance step is chosen first.

Overriding and multiple inheritance may each cause non-monotonic behavior. A relation of logical entailment $\Gamma \approx \Gamma$ is called non-monotonic if for some $\mathbf{P} \Gamma \psi \Gamma$ and $\phi \Gamma$ it is possible that $\mathbf{P} \approx \phi$ and $\mathbf{P} \wedge$

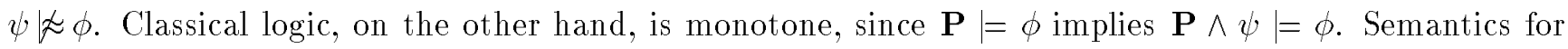
non-monotonic logics are usually much more involved $\Gamma$ and the corresponding proof theories are rarely complete.

To see why overriding may cause non-monotonic behaviorTconsider the example in $(20)$ Tand let $\approx$ be the logical entailment relation that does "the right thing" for in this situation. Let $\mathbf{P}$ denote the program in (20). Previously we have argued that the following is the intended inference:

$$
\mathbf{P} \quad \approx \quad \text { clyde }[\text { color } \rightarrow \text { white }]
$$

Now $\Gamma$ suppose we add $\psi=$ clyde[color $\rightarrow$ black $]$ to our set of assertions. Because of the overriding $\Gamma$ the property color $\rightarrow$ white is no longer inferred by clyde and $\Gamma$ thus $\Gamma \mathrm{P} \wedge \psi \not \notin$ clyde[color $\rightarrow$ white].

Multiple inheritance causes non-monotonic behavior for similar reasons. Consider a part of the Nixon's Diamond (21)Twhere it is not yet known that Nixon is a Quaker. In that case nixon would inherit policy $\rightarrow$ hawk from the only classTrepublican Twhere it belongs. HoweverTin a full-blown Nixon's

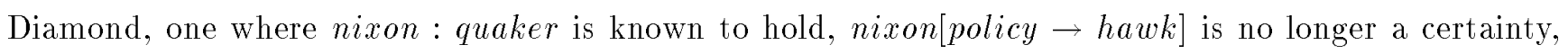
but rather just one of two possibilities.

Nonmonotonic inheritance has been a subject of intensive research (seeГe.g.Г[36Г 91 Г $92 \Gamma 43 \Gamma 22 \Gamma 90 \Gamma$ 57 [38Г56[80]). The main difference between our approach and the above works is that we are developing an inheritance theory for a general-purpose object-oriented language. By contrast Tmost of the aforesaid papers tend to study inheritance from a very general $\Gamma$ philosophical standpoint. This difference in the orientation clearly shows in the languages they use. First $\Gamma$ these languages are mostly propositional and TimportantlyГdo not distinguish between properties (i.e. TattributesTin our terminology) and classes. Second $\mathrm{T}$ these languages are not part of a more general logic programming system $\mathrm{Tand}$ so they do not raise the difficult issue of the interaction between inheritance and the ordinary logical deduction.

The semantics for inheritance that will be developed in this section is of the credulous breed [43] $\Gamma$ which means that in the presence of an inheritance conflict we are willing to accept multiple canonical models. This phenomenon can be interpreted in two different ways. One way to look at this is to view 
Here clyde is a member of the class royalElephant and so it inherits color $\bullet$ white from that class (which turns into a non-inheritable data expression $\Gamma$ color $\rightarrow$ white). In principle $\Gamma i t$ could have inherited color $\bullet$ grey from the class elephant. However $\Gamma$ a more specific class of clyde $\Gamma$ royal Elephant $\Gamma$ has an overriding inheritable property color $\bullet$ white. Likewise $\Gamma$ royalElephant does not inherit color $\bullet$ grey from elephant because of the aforesaid overriding property. On the other hand $\Gamma$ royalElephant inherits group $\bullet$ mammal as an inheritable property $\Gamma$ and clyde inherits it as a non-inheritable property group $\rightarrow$ mammal.

Note that (20) uses inheritable expressions to indicate that inheritance must take place. We remind from Section 3 that inheritable expressions are used in the following way: when such an expression is inherited by a class-member $\Gamma$ say clyde $\Gamma$ it becomes a non-inheritable expression within that member even if the member also plays the role of a class in some other context. In contrast Twhen an inheritable

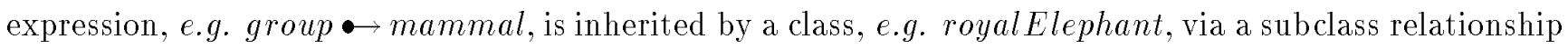
it remains an inheritable expression in that subclass $\Gamma$ and so it can be passed down to subclasses and members of that class (cf. the inheritance by royalElephant and by clyde).

To illustrate the rationale behind the dichotomy of inheritable/non-inheritable expressions $\Gamma$ we shall expand the example in Section 3 Twhere $b o b$ was represented as a member of the faculty.

Suppose that Tfor one reason or another Tthe information about bob's high school years and his years at Yale needs to be scrutinized. One way to represent this information is to create objects bobInHighschool and bobAt Yale and make them completely unrelated to the already existing object bob. The disadvantage of this approach is that then we will have to duplicate bob's date of birth $\Gamma$ gender $\Gamma$ etc. A better way is to turn the new objects into members of class bob as follows:

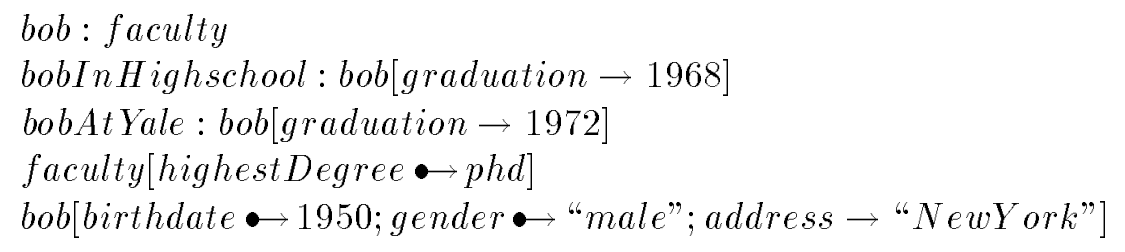

Here $b o b$ is a member of class faculty Twhile bobInHighschool and bobAtYale are members of bob. Additionally the second and the third clauses state that $b o b$ graduated from high school in 1968 and from Yale in 1972. Now bob[highestDegree $\rightarrow$ phd]. Notice that the property highestDegree $\bullet$ phd inherited from faculty turns into a non-inheritable property of $b o b \Gamma$ since inheritable properties are passed down to class members as non-inheritable properties. Because of that ThighestDegree $\rightarrow$ phd can no longer be propagated to the

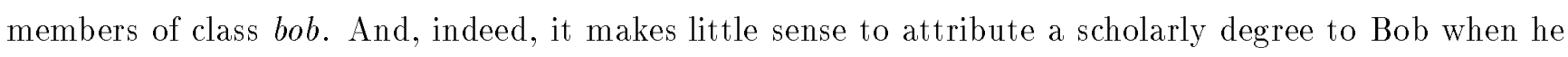
was in high school or a student at Yale. Likewise Tthe property address $\rightarrow$ "NewYork" of bob should not

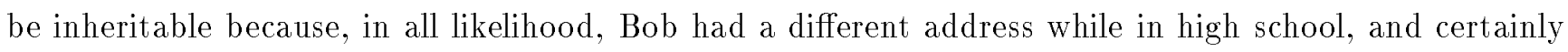
a different address while at Yale. On the other hand properties $\Gamma$ because these characteristics are not likely to change with time.

The phenomenon of multiple inheritance can be illustrated with the following example that is com- 
Other useful encapsulation policies can be represented along similar lines. We should mention that the above definition of type correctness has the same drawback as the notion presented in Section 12: being purely semantic $\Gamma$ it is weaker than desired. For instance $\Gamma$ suppose that $\Gamma$ no faculty has a recorded birthdate in the database. Then adding the clause (19) to faculty would still yield a well-typed program $\Gamma$ until a faculty with a proper birth date is inserted. Following this updateTthe program would become ill-typedTand in this way the use of the person-private method birthdate is precluded inside the module faculty. It should be clearThoweverTthat this weakness comes from our notion of type correctness-it is not inherent in our treatment of encapsulation.

\section{Inheritance}

The concept of inheritance is fundamental in AI and in object-oriented programming Tand a number of researchers had worked on combining this idea with programming languages. There are two main aspects of inheritance: structural inheritance and behavioral inheritance. Structural inheritance is a mechanism for propagating method declarations from superclasses to their subclasses. On the other hand Tbehavioral inheritance propagates what methods actually do rather than how they are declared.

\subsection{Structural Inheritance}

Structural inheritance is a subject of many works in functional and logic languages. Cardelli [25] considers inheritance in the framework of functional programming. He described a type inference procedure that is sound with respect to the denotational semantics of his system. Sound type inference systems for functional languages were also discussed in [74Г33] and in several other papers.

LOGIN and LIFE [6Г7] incorporate structural inheritance into logic programming via a unification algorithm for $\psi$-terms - complex structures that are related to signatures in F-logic but are different $\Gamma$ both semantically and syntactically.

In contrast to the above worksTF-logic is a full-fledged logic in which structural inheritance is built into the semanticsTand the proof procedure is sound and complete with respect to this semantics. Structural inheritance was discussed in Section 7.3.

\subsection{Behavioral Inheritance}

The main difficulty in dealing with behavioral inheritance is the fact that it is non-monotonic Twhich is mostly due to the property called overriding and also because of the phenomenon of multiple inheritance.

Overriding means that any explicit definition of a method takes precedence over any definition inherited from a superclass. For instanceTconsider the following F-program:

$$
\begin{array}{ll}
\text { royalElephant }:: \text { elephant } & \text { elephant }[\text { color } \bullet \text { "grey"; group } \bullet \text { mammal }] \\
\text { clyde }: \text { royalElephant } & \text { royalElephant }[\text { color } \bullet \text { "white"] }
\end{array}
$$


(i) both signatures are annotated with the same keyword; ${ }^{23}$ and

(ii) if the annotation is private or export-toTthen the rules $\rho_{1}$ and $\rho_{2}$ belong to one module.

A module structure is coherent if it is coherent with respect to all canonic H-models of $\mathbf{P}$.

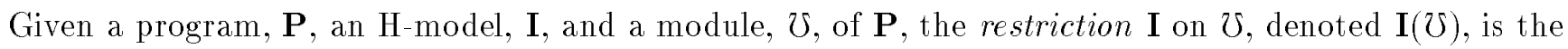
smallest H-structure that contains the following atoms:

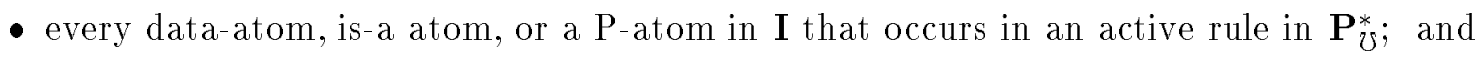

- every signature atom in $\mathbf{I}$ that is:

- a publicly annotated signature occurring in the head of an active rule in $\mathbf{P}^{*}$; or

- a privately annotated signature that occurs in the head of an active rule in $\mathbf{P}_{\mho}^{*}$; or

- a signature explicitly exported into $\mho$ from another module $\mho^{\prime}$ Tand such that it occurs in the head of an active rule in $\mathbf{P}_{\mho}^{*}$.

In other words $\mathrm{I}(\mho \mho)$ takes all data atoms that are relevant to the module and all signature atoms that are either derivable within that module or are exported by other modules. For instance $\mathrm{T}$ the restriction on administrative of a canonic model of a program that includes the three modules (16) - (18) will contain all data atoms that appear in the active instances of the last four clauses in (18) Tall the signatures in that

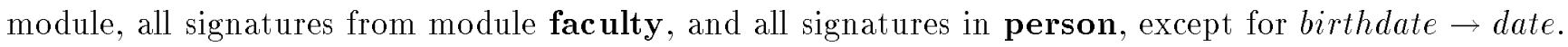

We can now define the notion of type-correctness under meta-annotations as follows:

Definition 13.3 (Type-Correctness with Meta-Annotations) Let $\mathbf{P}$ be an F-program with a coherent module structure. Then $\mathbf{P}$ is said to be type-correct (or well-typed) if it is well-typed in the usual sense (with respect to Definition 12.5) and if every module of $\mathbf{P}$ is also well-typed.

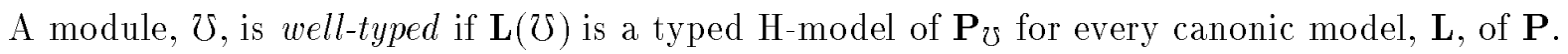

To illustrate this definition $\Gamma$ consider $\Gamma$ again $\Gamma$ the module structure (16) - (18). This program is well-typed because all signatures used in these modules are properly exported. Suppose now that we add

$$
F[\text { birthmonth } \rightarrow M] \leftarrow F: \text { faculty }[\text { birthdate } \rightarrow d(D, M, Y)]
$$

to module faculty. The resulting program will not be type-correct Tbecause the module faculty will no longer be well-typed.

To see whylet $\mathbf{L}$ be a canonic model of this program. The restriction $\mathrm{L}$ (faculty) does not contain the signature faculty[birthdate $\Rightarrow$ date] [since the latter does not appear in the head of any active rule local to faculty $\Gamma$ and it is not exported into this module. On the other hand $\Gamma$ if there is even a single facultyГsay fred Tfor whom birthdate is recorded Tthen fred[birthdate $\rightarrow \cdots]$ will be in $\mathrm{L}$ (faculty). Since this data atom is not covered by any signature in $\mathrm{L}$ (faculty) Tthe latter is not a typed H-structure.

\footnotetext{
${ }^{23}$ export-to(a) and export-to(b) are considered to be the same keyword for this purpose.
} 
(ii) An annotation (privateTpublicTor export-to(module)) attached to each signature expres-

sion that occurs in the head of a rule in $\mathbf{P}$.

Note that since modules do not necessarily consist of disjoint sets of clauses $\Gamma$ one module may be a sub-module of another module. Note further that $\Gamma$ as illustrated above $\Gamma$ some modules may represent authorization domains for various classes of users. Clauses and queries introduced by these users are assumed to enter the appropriate modules automatically. AlsoT Definition 13.1 does not assume that there is a correspondence between modules and classes; however Tif desiredTmodules can be made to fit

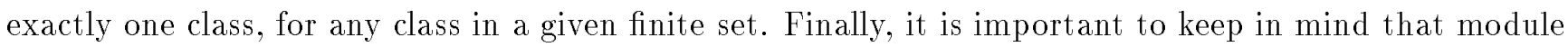
structures do not affect the canonic model semantics of the program-only the notion of type correctness is affected Tas explained below.

The above definition of module structures is very general and calls for further specialization. One thing that immediately comes to mind is that certain module structures may have internal contradictions. For instanceГif a method is declared as public in one module and as private in anotherTa problem may arise if these declarations clash. We consider a module structure with this property as incoherent.

Another situation where a module structure may be viewed as incoherent is when the same method expression is declared as private in two separate modules. If this were allowed Tany module could import any signature by declaring it private. This policy howeverTcannot control what is being exported and wheretoTand thus it prevents enforcing any sensible access authorization policy. There are also purely software-engineering arguments against this policyГwhich has led many object-oriented systems to adopting the idea of controlled export rather than import. A consequence of this policy is that duplication of private declarations in different modules is not allowed. Similarly we shall assume that a signature can be exported from only one module-the module where it is declared. These ideas are formally captured via conditions (i) and (ii) of Definition 13.2Tbelow.

To formulate the notion of coherence $\Gamma$ we need a few simple definitions. Let $\mathcal{L}$ be an F-logic languageI $\mathbf{P}$ be a program with a module structure Tand let $\mho$ be a module of $\mathbf{P}$. Let $\mathbf{P}_{\mho}$ denote the set of clauses in $\mathbf{P}$ that belong to $\mho$. The notation $\mathbf{P}^{*}$ (or $\mathbf{P}_{\mho}^{*}$ ) will be used to denote the set of annotated ground

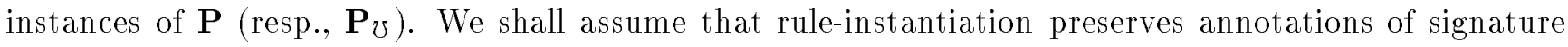
expressions occurring in the heads of the rules in $\mathbf{P} \Gamma$ and that instances of the signatures that have different annotations are not merged..$^{21}$

Let $\mathbf{I}$ be an $\mathrm{H}$-structure of $\mathbf{P}$. (Note: $\mathbf{I}$ has no annotationsTbut $\mathbf{P}$ and $\mathbf{P}^{*}$ have). A ground rule in $\mathbf{P}^{*}$ is active in $\mathbf{I}$ if all its premises are true in $\mathbf{I}$. If $\mathbf{I}$ is a model $\Gamma$ then the heads of all active rules are also true in $\mathrm{I}$.

Definition 13.2 (Coherent Module Structures) A module structure for $\mathbf{P}$ is coherent with respect to a canonic $\mathrm{H}$-modelГ LTif for every data-atom in $\mathbf{L}$ that is covered $^{22}$ by a pair of signatures that occur in the heads of some active rules $\Gamma \rho_{1}, \rho_{2} \in \mathbf{P}^{*}$ (where $\rho_{1} \equiv \rho_{2}$ is possible) $\Gamma$ the following holds:

\footnotetext{
${ }^{21}$ For instance, instantiating $X$ and $Y$ to $b$ in $a[$ private $: X \rightarrow c$; public $: Y \rightarrow c]$ yields $a[$ private $: b \rightarrow c$; public: $b \rightarrow c]$, where the two identical instances of $b \rightarrow c$ are kept separately due to the difference in annotations.

${ }^{22}$ Covering is defined in Definitions 12.1 and 12.2 .
} 
The above represents a fragment of the definition of the module faculty. The methods account and balance are not visible by any module except faculty and a module where administrative workers process project accounts. In this particular case $\mathrm{Cthe}$ reason for shielding account and balance is that of protection rather than encapsulation - we assume that the expenditures in project accounts are not in the public domain. However Tthe total amount of funds initially set aside for the project is made available through the public method funding.

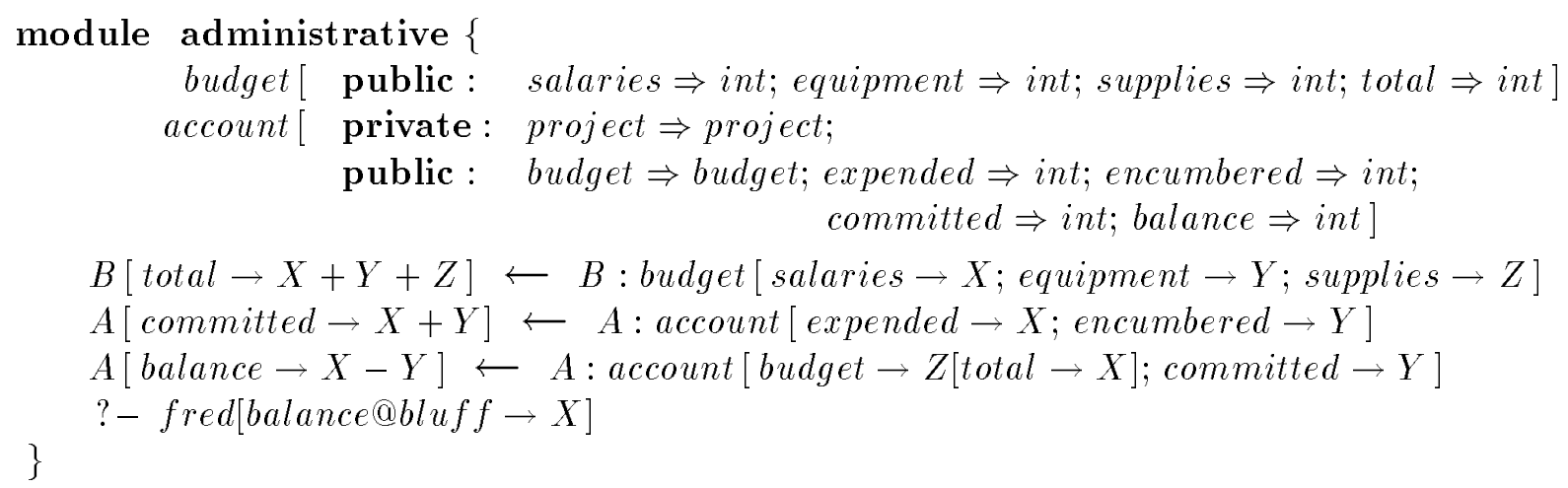

Unlike module person $\Gamma$ the last two modulesTadministrative and faculty $\Gamma$ are not built around any specific class. Apart from being depositories for method definitions Tthese modules serve as authorization domains for the members of the faculty and for workers who administer project accounts. Note that faculty exports two methods to module administrative $\Gamma$ which makes their invocation in that module legal (cf. the last query in administrative). AlsoTalthough most of the information about an account is made public in module administrative $\Gamma$ the identifying information of the account is not $\Gamma$ which is achieved by making the attribute project in class account (project associated with account) private.

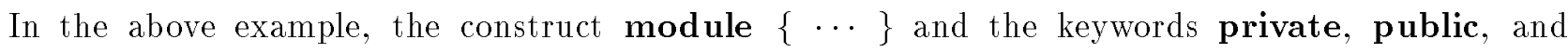
export-to are not part of the logic but $\Gamma$ rather $\Gamma$ are meta-annotations. Their purpose is to affect the notion of type correctness presented in Section 12 Wwich itself is a meta-logical concept. The following section gives a formal account of type-correctness for programs with a module structure.

\subsection{Modules and Type Correctness}

The above example contains all major elements of our treatment of encapsulation. The key idea is to use a stronger notion of type-correctness to preclude illegal uses of methods with limited scope. This requires imposition of a module structure on each F-program $\Gamma$ which is specified via meta-logical annotations. The new notion of type correctness builds on the notion introduced in Section 12 and reduces to the old notion when the entire F-program is considered as one big module.

Definition 13.1 (Module Structures) Let $\mathbf{P}$ be an F-program. A module structure of $\mathbf{P}$ consists of the following:

(i) A decomposition of the set of clauses in $\mathbf{P}$ into a collection of named (not necessarily disjoint) subsetsTcalled modules; 
First $\Gamma$ all program clauses must be grouped into modules. Then each module is checked for typecorrectness separately. The notion of type-correctness referred to here is more elaborate than the one in Section 12. The main difference is that the set of signatures used in determining type-correctness of any given module now consists not only of signatures determined by the clauses that belong to that module but also of signatures that are exported to this module by other modules. ${ }^{20}$

It should be noted that in most object-oriented systemsTencapsulation policies center around the concept of a class $\Gamma$ not module. However $\Gamma$ in a language where classes can be virtual $i . e . \Gamma$ defined via logical clauses Tencapsulating each class seems to be infeasible. Nevertheless Tmodules can be made to fit exactly one class andTthus They provide a way to encapsulate any fixed number of classes (which is all what current systems are capable of doing Tanyway).

TraditionallyTobject-oriented systems were employing the idea of encapsulation for hiding implementation details $\Gamma$ and our approach is not different in this respect. However $\Gamma$ a combination of modules with type-correctness can serve another useful purpose: authorization-based control over access to objects. This can be achieved along the following lines. FirstTeach user is assigned to a module. Then $\Gamma$ each clause or a query that a user adds to the system is automatically made part of that user's module. This simple schema can control unauthorized access $\Gamma$ because any user query would constitute a type error (and would be rejected) if it were to invoke a method that is not exported into that user's module.

\subsection{An Example}

To illustrate the above ideasT consider a program consisting of the following three modules:

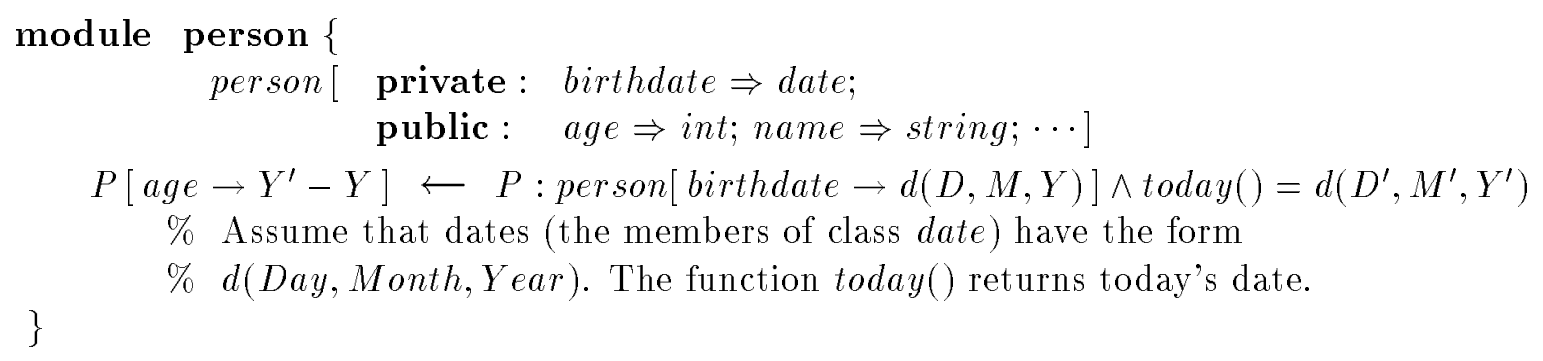

In this module $\Gamma$ the attribute birthdate is declared as private in order to encapsulate the implementation of the method age from future changes to the structure of the class person. For instance Twe may replace birthdate by yearOfBirth $\Gamma$ or we may change the type of birthdate without impacting methods defined outside the module person.

module faculty \{

$$
\begin{aligned}
& \text { faculty [ public: funding@project } \Rightarrow \text { int; projects } \Rightarrow \text { project; } \cdots \text { ] } \\
& \text { project[ export-to(administrative): } \\
& \text { account } \Rightarrow \text { account; balance@project } \Rightarrow \text { int; } \cdots] \\
& F[\text { funding } @ P \rightarrow X] \leftarrow F: \text { faculty }[\text { projects } \longrightarrow \\
& P[\text { account } \rightarrow A[\text { budget } \rightarrow B[\text { total } \rightarrow X]]]] \\
& F[\text { balance } @ P \rightarrow X] \leftarrow F: \text { faculty }[\text { projects } \rightarrow P[\text { account } \rightarrow A[\text { balance } \rightarrow X]]] \\
& \text { \} }
\end{aligned}
$$

\footnotetext{
${ }^{20}$ Public signatures are considered to be exported to every module.
} 
with restricted classes of F-programsTe.g. [42]). On the other handTwhile Definition 12.5 accommodates a vast class of programsTit is weaker than we would like it to be. EssentiallyTour notion of type-correctness amounts to saying that well-typed programs are not allowed to derive facts that are inconsistent with signatures specified for these programs. Clearly this is a minimum that must be required of a correct logic program. However Tanother source of type errors comes from rules that have signature-incompatible body-literals. Unfortunately this latter kind of errors is not captured by Definition 12.5. A detailed discussion of these issues and some solutions can be found in [98Г29].

The semantics of type-correctness presented here is of the type-checking variety which means that it requires the programmer to supply a complete type specification for each program. HoweverTin logic programming $\Gamma$ supplying complete type specifications is often viewed as a bother. Adapting the ideas from ML to PrologTMishra [73] proposed to use type-inference for determining predicate types and then use the inferred types for trouble-shooting logic programs.

Semantics of type inference is different from the semantics of type checking. Briefly $\mathbf{P}$ Tits inferred type is the "strongest" type declaration under which $\mathbf{P}$ is well-typed. If pure type-checking is viewed by some as too taxing Tpure type-inference appears to be at another extreme and so it is used mostly as an advisory tool. We feel — as suggested by other researchers in the past [101Г99] - that typeinference combined with type checking is a suitable compromise. A way to define logical semantics for this approach was proposed in [51Г98].

\section{Encapsulation}

Encapsulation is a major concept in the suite of notions comprising the object-oriented paradigm. In a nutshell encapsulation is a software-engineering technique that requires special designations for each method in a class. Methods designated as public can be used to define other methods in other classes. In contrast Tmethods designated as private can be used only in the class where they are declared. Other methods may be exported to some specific classesTbut not to all classes.

While the idea of encapsulation is simple Tits logical rendition is not. In [71] CMiller proposes a way to represent modules in logic programming via the intuitionistic embedded implication. Chen [28] defines modules as second-order objects Twhere data encapsulation inside modules is represented using existential quantifiers. In their present form $\Gamma$ these methods are not sufficiently general to be applied to F-logic. It would be interesting to see $\mathrm{T}$ though $\Gamma$ if these approaches can be extended to make them suitable for method encapsulation in an object-oriented logic.

In contrast to [71Г28] Twe view various encapsulation mechanisms simply as elaborate type-correctness policies. This approach is quite general and is capable of modeling a wide variety of encapsulation mechanisms. Its other advantage is that it does not require extension to the logic. Insteadrencapsulation is treated as a type-correctness discipline and $\Gamma$ thus $\Gamma i t$ is a meta-logical notion in our approach. ${ }^{19}$

The general outline of our encapsulation-as-type-correctness approach can be formulated as follows.

\footnotetext{
${ }^{19}$ An interesting feature of $[71,28]$ is that they achieve encapsulation by purely logical means.
} 
This model satisfies the well-typing conditions of Definition 12.3 and Therefore $\mathrm{Tis}$ a typed F-structure. HenceГP is well-typed.

Suppose now that $\mathbf{P}$ contains the term mary[boss $\rightarrow$ john $]$ instead of mary[boss $\rightarrow$ bob $]$. Then $\mathbf{P}$ would have had a type error because the atom mary[boss $\rightarrow$ john] clashes with the typing empl[boss $\Rightarrow$ faculty]. Indeed $\Gamma$ john is not known to be a faculty and hence $\neg$ john : faculty holds in every canonic model of $\mathbf{P}$. Likewise $T$ the clause mary[salary $\rightarrow 10000]$ would have caused a type error. This happens because no signature was specified for the 0-ary version of the method salary (only the unary version of salary has a signature in $\mathbf{P})$ Tand so $\neg$ faculty[salary $\Rightarrow()]$ and $\neg$ empl[salary $\Rightarrow()]$ must hold in the minimal model of $\mathbf{P}$. But this $\Gamma$ together with mary $[$ salary $\rightarrow 10000]$ C defeats the first well-typing condition of Definition 12.3 .

For another exampleTconsider:

$$
\begin{aligned}
& i b m: \text { company } \\
& \text { john }[\text { worksFor } \rightarrow i b m] \\
& X: \text { employee } \leftarrow X[\text { worksFor } \rightarrow I] \\
& X[\text { worksFor } \Rightarrow \text { company }] \leftarrow X: \text { employee }
\end{aligned}
$$

This F-program is well-typed because its minimal model is also a typed H-model. In contrast Tif the last rule in (15) were

$$
X[\text { name } \Rightarrow \text { string }] \longleftarrow X: \text { employee }
$$

the program would not be well-typed because the attribute worksFor would have had no declared signature andThence $\neg$ c[worksFor $\Rightarrow()]$ would hold in that model $\Gamma$ for every class $c$. ThisTtogether with john [worksFor $\rightarrow i b m]$ Tdefeats the first well-typing requirement to typed H-structures (Definition 12.1). Therefore Ceven though the modified program has a minimal model it is not a typed model and so the program is ill-typed.

\section{Discussion}

Definition 12.5 provides semantic grounds for developing static type-checking algorithms. It also supplies a yardstick for verifying correctness of various such algorithmsTand thus provides a uniform framework for comparison. Static type checking is a topic for further research; for classical logic programs defined notion of type-correctness was studied in [98]. A detailed comparison of our approach with other works on type systems for logic programming can be found in [51Г98].

To better see the role of type correctness in the overall schema of things is not part of F-logic butTratherTbelongs to its meta-theory. As such $\Gamma$ neither the semantics $\Gamma$ nor the proof theory depends on the particular way well-typing is defined. Even the sublogic of signatures is independent of Definition 12.5. ThereforeTother definitions of type-correctness can also be used in conjunction with F-logic.

The need for a new notion of type-correctness for F-logic arose because existing theories are not general enough to adequately type many kinds of F-programs (although some could be adapted for use 
that there should be a signature defined for some class that contains faculty as a member and that covers the invocation faculty[avgSalary $\rightarrow 50000] .{ }^{18}$

Definition 12.2 (Typed H-structures-Inheritable Data Expressions) Let I be an H-structure. If $\alpha$ is an inheritable data atom of the form $c\left[m @ a_{1}, \ldots, a_{k} \curvearrowleft v\right] \in \mathbf{I}$ and $\beta$ is a signature atom of the form $d\left[m @ b_{1}, \ldots, b_{k} \approx \cdots\right] \Gamma$ where $\bullet$ and $\approx$ standTrespectivelyTfor $\bullet$ and $\Rightarrow$ or for $\bullet$ and $\Rightarrow$ Twe shall say that $\beta$ covers $\alpha$ if $a_{i}: b_{i}, c:: d \in \mathrm{I}$.

An H-structure $\mathrm{I}$ I is said to be typed with respect to inheritable data expressions if the following conditions hold:

- Every inheritable data atom in $\mathbf{I}$ is covered by some signature in $\mathbf{I}$; and

- If an inheritable data atom $\Gamma c\left[m @ a_{1}, \ldots, a_{k} \bullet \sim v\right] \in$ I $\Gamma$ is covered by a signature of the form $d\left[m @ b_{1}, \ldots, b_{n} \approx w\right] \in \mathrm{I} \Gamma$ where $v, w \in U(\mathcal{F})$ and $\rightsquigarrow$ and $\approx$ are as before $\Gamma$ then $v: w \in \mathrm{I}$.

Definition 12.3 (Typed H-Structures - All Data Expressions) The four conditions in Definitions 12.1 and 12.2 are called the well-typing conditions. An H-structureГІГ is typed if all well-typing conditions are satisfied $\Gamma$ i.e. Tif $\mathbf{I}$ is typed with respect to inheritable and non-inheritable data expressions.

We are now ready to define the notion of well-typed F-programs:

Definition 12.4 (Typed Canonic Models) A typed canonic model of $\mathbf{P}$ is a (usual) canonic model for $\mathbf{P}$ that $\Gamma$ in addition $\Gamma$ is a typed H-structure.

Definition 12.5 (Well-typed Programs) An F-program $\mathbf{P}$ is well-typed (or type-correct) if every canonic $\mathrm{H}$-model of $\mathbf{P}$ is a typed canonic H-model. Otherwiser $\mathbf{P}$ is said to be ill-typed.

This definition appears to capture the intuition about type errors quite adequately: If $\mathbf{P}$ has a nontyped canonic model $\Gamma$ the problem can clearly be traced to typing. For example $\Gamma$ consider the following F-programГP:

$$
\begin{array}{lll}
\text { bob }: \text { faculty } & 1989: \text { year } & \text { empl[boss } \Rightarrow \text { faculty; salary } @ \text { year } \Rightarrow \text { integer }] \\
\text { mary }: \text { faculty } & 10000: \text { int } & \text { mary }[\text { boss } \rightarrow \text { bob }] \\
\text { faculty }:: \text { empl } & &
\end{array}
$$

The minimal H-model of $\mathbf{P}$ consists of the following molecules (and their derivatives):

$$
\begin{array}{lcc}
\text { faculty }:: \text { empl } & \text { bob }: \text { faculty } & 1989: \text { year } \\
\text { bob }: \text { empl } & \text { mary }: \text { faculty } & 10000: \text { int } \\
\text { mary }: \text { empl } & \text { mary }[\text { boss } \rightarrow \text { bob }] & \\
\text { empl[boss } \Rightarrow \text { (faculty,empl }) ; \text { salary } @ y e a r \Rightarrow \text { integer }] \\
\text { faculty[boss } \Rightarrow \text { (faculty,empl); salary@year } \Rightarrow \text { integer }]
\end{array}
$$

\footnotetext{
${ }^{18}$ Such a class, called employmentGroup was introduced towards the end of Section 3.
} 
attributes that are defined for the particular relation at handTand any attempt to use an attribute that is not defined for this relation will normally result in an error.

To illustrate we reproduce an example from Section 11.3:

$$
\begin{aligned}
& \text { empl }[\text { salary } @ \text { year } \Rightarrow \text { integer }] \\
& \text { person }[\text { birthdate } \Rightarrow \text { year }]
\end{aligned}
$$

Here we would like to make it illegal for an empl-object to call methods other than birthdate and salary. Similarly $\Gamma$ for persons that are not employees $\Gamma$ we would like to prohibit the use of the attribute salary altogetherГsince it is not covered by an appropriate signature in class person. In other wordsTwe would like to say that (in the above example) the signature of person-objects consists precisely of one method $\Gamma$ birthdate Tand that the signature of empl-objects has precisely two methodsTsalary and birthdate. Both methods are scalar; birthdate expects no argumentsTwhile salary needs one-a year-object.

Unfortunately by itself these declarations do not ensure that the corresponding typing constraint hold in a canonic $\mathrm{H}$-model $\mathrm{Cbecause}$ connection is missing between signatures and data expressions. This missing link is now provided in the form of the well-typing conditions:

Definition 12.1 (Typed H-structures-Non-inheritable Data Expressions) Let $\mathbf{I}$ be an H-structure. If $\alpha$ is a non-inheritable data atom of the form $o\left[m @ a_{1}, \ldots, a_{k} \sim v\right] \in \mathrm{I}$ and $\beta$ is a signature atom of the form $c\left[m @ b_{1}, \ldots, b_{k} \approx \cdots\right]$ Twe shall say that $\beta$ covers $\alpha$ ifT for each $i=1, \ldots, k$ Twe have $o: c, a_{i}: b_{i} \in \mathrm{I}$. Here

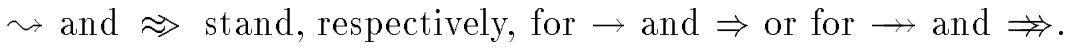

We shall say that $\mathbf{I}$ is a typed $\mathrm{H}$-structure with respect to non-inheritable data expressions if the following conditions hold:

- Every non-inheritable data atom in $\mathbf{I}$ is covered by a signature atom in $\mathbf{I}$; and

- If a data atom $\Gamma o\left[m @ a_{1}, \ldots, a_{k} \sim v\right] \in$ ITis covered by a signature of the form $c\left[m @ b_{1}, \ldots, b_{n} \approx w\right] \in$ ITwhere $\sim$ and $\approx$ are as before and $v, w \in U(\mathcal{F}) \Gamma$ then $v: w \in \mathbf{I}$.

Note that the first condition imposes restrictions on the domain of the definition of methods $\Gamma$ and on when a method can be invoked as a scalar or a set-valued method. In plain terms non-inheritable expression in I must be covered by a signature. The second condition says that every noninheritable expression must satisfy all constraints imposed by the signatures that cover that expression.

When it comes to typing $\Gamma$ inheritable expressions are not completely analogous to non-inheritable expressions Tdespite their semantic and proof-theoretic similarities. The reason for this is that inheritable expressions are used differently. Indeed $\Gamma$ consider the expression highestDegree $\bullet$ phd of class faculty in Section 3. This expression is supposed to be inherited by the members of class faculty and thus it is also a property of these members. Therefore

It is instructive to compare the above expression to avgSalary $\rightarrow 50000$ Twhich is a non-inheritable property of faculty. Since this expression does not apply to the members of the classTit does not have to comply with the signatures given for the class faculty. Instead DDefinition 12.1 applies hereTwhich means 
This part of the job is quite standard and can be found in many guides to Prolog. Once the expression

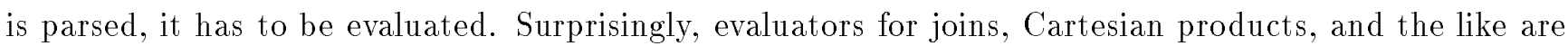
rather cumbersome and take anywhere from one to two pages of Prolog code. The main difficulty here is the fact that arities of the expressions to be joined are unknown at compile time $\mathrm{which}$ calls for the use of various nonlogical features such as "=.." $\Gamma$ "arg" Tand "functor". We shall see that a page-plus-long Prolog program for the relational join evaluator can be written in just three rules in F-logic.

Relations are represented as in Section 6 Th that is $\Gamma$ each relation $\Gamma p \Gamma$ is a class of tuple-objects. Given a pair of relations $\Gamma p$ and $q$ Tand a join condition $\Gamma$ cond $\Gamma$ the program creates a new relation $\Gamma j o i n(p, q, c o n d)$. Join-conditions are represented as lists of pairs of the form $\operatorname{cond}(A, B, \operatorname{cond}(\ldots))$. For instance $\operatorname{cond}\left(A_{1}\right.$, $B_{1}, \operatorname{cond}\left(A_{2}, B_{2}\right.$, nil $\left.)\right)$ encodes an equi-join condition $A_{1}=B_{1} \wedge A_{2}=B_{2}$. The first deductive ruleTbelow says that if $i$ and $j$ are oid's of tuples in relations $p$ and $q$ Trespectively $\Gamma$ then $n e w(i, j)$ is the oid of a tuple in a nil-join of $p$ and $q$ (i.e. Tin a Cartesian product of $p$ and $q$ ). The second rule recursively computes the oid's of all tuple-objects in the equi-join. These tuple-objects are not fully defined $\Gamma$ however $\Gamma$ as we have not yet specified the values of their attributes. The third ruleTtherefore is needed to extract these values from the source-tuples $i$ and $j$ :

$$
\begin{aligned}
& \operatorname{new}(I, J): \operatorname{join}(P, Q, \text { nil }) \leftarrow \quad I: P \wedge J: Q \\
& \operatorname{new}(I, J): \operatorname{join}(P, Q, \operatorname{cond}(L, M, \text { Rest })) \leftarrow \quad I: P[L \rightarrow X] \wedge J: Q[M \rightarrow X] \\
& \wedge \operatorname{new}(I, J): \operatorname{join}(P, Q, \text { Rest }) \\
& \operatorname{new}(I, J)[L \rightarrow X, \operatorname{rename}(M) \rightarrow Y] \leftarrow \quad I: P[L \rightarrow X] \wedge J: Q[M \rightarrow Y]
\end{aligned}
$$

Note that in the last rule the attributes coming from the second relation in the join are renamed in order to avoid name clashes. When relations do not have common attributes or when we are interested in natural joins rather than equi-joins This renaming would not be needed and so the symbol rename in the last rule could be dropped. Additionally for natural joins $\Gamma$ the join-condition itself can be simplified to be a list of attributes instead of pairs of attributes.

Now $\Gamma$ if dept has attributes dname and mngr $\Gamma$ while the relation empl has attributes dname and ename $\Gamma$ the query ? - $X: j o i n(d e p t, e m p l$, cond(dname,dname, nil $))[Y \rightarrow Z]$ will return a join of the two relations on the attribute dname.

Actually the above program does a more general job since it joins classes rather than just relations. It also demonstrates a difference that syntax can make: In Prolog Ta purely logical specification of a join entails a rather sophisticated encoding of relations Twhich - in the end-results in a code that can hardly be called "declarative."

\section{Well Typed Programs and Type Errors}

In a strongly typed language $\Gamma$ a method can be invoked only if this invocation is covered by one of the

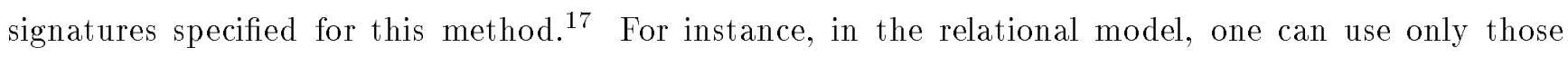

\footnotetext{
${ }^{17} \mathrm{By}$ an invocation we mean a tuple of the form $\langle o b j, \overrightarrow{a r g} s\rangle$, where $o b j$ is the host-object of the invocation and $a \overrightarrow{r g} s$ is a list of arguments of the invocation.
} 
of a general situation where joint projectsTcommon hobbiesTand other commonalities may be involved. We can abstract this concept and define a "generic" method for joint things:

$$
\begin{aligned}
X[j \operatorname{joint}(M) @ \operatorname{nil} \rightarrow Z] & \leftarrow X[M \rightarrow Z] \\
X[j \operatorname{joint}(M) @ \operatorname{cons}(O b j, R e s t) \rightarrow Z] & \leftarrow O b j[M \rightarrow Z] \wedge X[j \operatorname{joint}(M) @ \operatorname{Rest} \rightarrow Z]
\end{aligned}
$$

The first rule here describes things that $X$ has in common with an empty list of objectsTnil. The second rule Tthen Tsays that $X$ has object $Z$ in common with each member of the list cons (Obj, Rest) if $O b j$ has $Z$ and $Z$ is a common object that $X$ shares with the rest of the list.

Our second example describes similarities among classesTspecified via the like-relationship (cf. [41T 11]). For instance $\Gamma$ one can say "The concept of a Whale is like the concept of a Fish via habitat" or $\Gamma$ "A Pig-Like-Person is like a Pig via nose and legs."

Like-similarity can be expressed by means of a ternary predicateTlike. For instanceTin like(pig-like, pig, cons(legs, cons(nose, nil)) ) T the third argument would list the attributes that relate the first two arguments. We could then define:

$$
C_{1}[\text { Attr } \rightarrow P] \leftarrow C_{2}[\text { Attr } \rightarrow P] \wedge \operatorname{like}\left(C_{1}, C_{2} \text {,PropList }\right) \wedge \text { member }(\text { Attr, PropList })
$$

where member is a membership predicate that can be defined by a standard Prolog program.

The above technique works well for specifying similarity via attributes $\Gamma$ i.e. Tvia 0 -ary methods. To represent similarity via methods of arbitrary arities we would need one like $e_{n}$ predicate for each arity $n \geq 0$ Tand one rule of the form

$$
\begin{aligned}
C_{1}\left[M @ X_{1}, \ldots, X_{n} \rightarrow P\right] \leftarrow C_{2}\left[M @ X_{1}, \ldots, X_{n} \rightarrow P\right] & \wedge \text { like }_{n}\left(C_{1}, C_{2}, \text { MethdList }\right) \\
& \wedge \text { member }(M, \text { MethdList })
\end{aligned}
$$

\subsubsection{List Manipulation}

The following is an F-logic counterpart of a canonic textbook example:

$$
\begin{aligned}
& n i l[\text { append }[\rightarrow L] \leftarrow L: \operatorname{list}(T) \\
& \operatorname{cons}(X, L)[\text { append } @ M \rightarrow \operatorname{cons}(X, N)] \leftarrow L: \operatorname{list}(T)[\text { append } @ M \rightarrow N] \wedge X: T
\end{aligned}
$$

Here $L \Gamma M \Gamma$ and $N$ are list-objects $\Gamma$ while append is a method that applies to list-objects; when invoked with a list-object $\Gamma$ $b$ as an argument on a list-object $\Gamma a \Gamma$ as a host append returns a concatenation of $a$

and $b$. A parametric family of list-classes $\Gamma$ list $(T) \Gamma$ and their signatures were defined earlier $\Gamma$ in $(10)$ of Section 11.2 and in (11) of Section 11.3 Trespectively.

\subsubsection{A Relational Algebra Interpreter}

Writing an intelligible Prolog interpreter for relational algebra is rather challenging. First Tone should parse the relational expression given as input. For exampleTan expression $((P \times V) \cup W) \bowtie_{\text {cond }}(Q-R)$ would be converted into a term of the form

$$
\text { join }(\text { union }(\operatorname{prod}(P, V), W), \operatorname{minus}(Q, R), \text { cond })
$$


Typically database queries are specified with respect to a fixed $\Gamma$ known scheme. Experience shows $\Gamma$ howeverThat this assumption is unrealistic and ad hoc schema exploration may often be necessary. This means that the user has to apply intuitive or exploratory search through the structure of the scheme and the database at the same time and even in the same query (cf. [76]). Many user interfaces to commercial databases support browsing to different extent. The purpose of the following examples is to demonstrate that F-logic provides a unifying framework for data and schema exploration. Once again Twe refer to the example in Section 3.

For each object in class faculty Tthe following pair of rules collects all attributes that have a value in class person:

$$
\begin{aligned}
& \text { interesting Attributes }(X)[\text { attributes } \rightarrow L] \leftarrow X: \text { faculty }[L \rightarrow Z: \text { person] } \\
& \text { interesting Attributes }(X)[\text { attributes } \rightarrow L] \leftarrow X: \text { faculty }[L \rightarrow Z: \text { person }]
\end{aligned}
$$

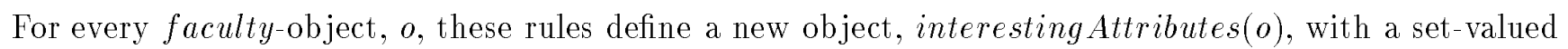
attribute attributes. The intuitive reading of (12) is: If $L$ is an attribute that is defined on an object $\Gamma$ $X \Gamma$ and has a person-value $Z \Gamma$ then $L$ must belong to the result that attributes has on the object interesting Attributes $(X)$.

Thus in the example in Section $3 \Gamma$ we would obtain: interestingAttributes $($ bob $)=\{$ boss $\}$ and interesting Attributes(mary) $=\{$ friends $\}$. Deleting the restriction person in (12) would add those attributes that have any value on $X$ Tnot just a person-object. In that case Cinteresting Attributes(bob) will also contain name Tage Tand worksFor Twhile interesting Attributes(mary) will include name ThighestDegreeTand worksFor.

Another interesting example is when we need to retrieve all objects that reference some other object directly or indirectly (via subobjects). The method find rbelow Treturns the set of all such objects that reference Stuff:

$$
\begin{aligned}
& \text { browser }[\text { find } @ \text { Stuff } \rightarrow X] \leftarrow X[Y \rightarrow \text { Stuff }] \\
& \text { browser }[\text { find } @ \text { Stuff } \rightarrow X] \leftarrow X[Y \rightarrow \text { Stuff }] \\
& \text { browser }[\text { find } @ \text { Stuff } \rightarrow X] \leftarrow X[Y \rightarrow Z] \wedge \text { browser }[\text { find } @ \text { Stuff } \rightarrow Z] \\
& \text { browser }[\text { find } @ \text { Stuff } \rightarrow X] \leftarrow X[Y \rightarrow Z] \wedge \text { browser }[\text { find } @ \text { Stuff } \rightarrow Z]
\end{aligned}
$$

For the example in Section $3 \Gamma$ the query ? - browser [find@ "CS" $\rightarrow X]$ would return the set $\left\{c s_{1}, c s_{2}, b o b\right.$, mary $\}$.

\subsubsection{Representation of Analogies}

Reasoning by analogy is an active field of research (e.g. Tsee a survey in [40]). Apart from the semantic and heuristic issues $\Gamma$ finding suitable languages in which to specify analogies is also a challenge. This subsection shows how certain kinds of analogies can be specified in F-logic.

In Section 3Twe defined a method that Twhen applied on a host object of type person with a personargument Treturns the set of all joint works of the two persons involved. This method is just one instance 
the other handTstudents may be entitled to see their own grades. To implement this policyГeach student can be given access to the object that represents the achievements of that student. We can then define the method grade on student-objects as follows:

$$
S t u d[\text { grade } @ C r s \rightarrow G] \leftarrow \text { Crs }: \text { course }[\text { grade } @ S t u d \rightarrow G]
$$

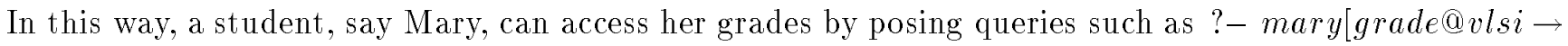

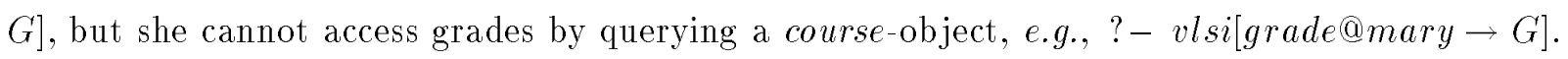

The power-set operator. Another interesting problem is to express the power-set operation $\Gamma$ an operator that takes an arbitrary set and constructs its power-set. First $\Gamma$ we define the class of sets; it consists of objects with oid's of the form $a d d(a, a d d(\ldots))$. The intended meaning of a set-object denoted by $\operatorname{add}(a, \operatorname{add}(b, a d d(c, n i l)))$ is the set $\{a, b, c\}$. The class of sets is defined as follows:

$$
\begin{aligned}
& n i l: \text { set } \\
& \operatorname{add}(X, S): \text { set } \leftarrow S: \text { set } \\
& \operatorname{add}(X, \operatorname{add}(Y, S)) \doteq \operatorname{add}(Y, \operatorname{add}(X, S)) \\
& \operatorname{add}(X, \operatorname{add}(X, S)) \doteq \operatorname{add}(X, S)
\end{aligned}
$$

The first equation asserts that the order of adding elements to sets is immaterial; the last equation says that insertion of elements into sets is an idempotent operation.

Next Twe define the attributeГself $\Gamma$ that for every set-object returns the set of all members of this set:

$$
\begin{aligned}
& \operatorname{nil}[\text { self } \rightarrow\{\}] \\
& \operatorname{add}(X, L)[\text { sel } f \rightarrow X] \leftarrow L: \text { set } \\
& \operatorname{add}(X, L)[\text { sel } \rightarrow Y] \leftarrow L: \operatorname{set}[\text { sel } f \rightarrow Y]
\end{aligned}
$$

Finally the powerset method is defined as follows:

$$
\begin{aligned}
& S[\text { powerset } \rightarrow \text { nil }] \quad \leftarrow S: \text { set } \\
& S[\text { powerset } \longrightarrow \operatorname{add}(Z, L)] \leftarrow S: \operatorname{set}[\text { self } \rightarrow Z \text {; powerset } \rightarrow L] \\
& \wedge L: \text { set } \wedge \neg L[\text { self } \longrightarrow Z]
\end{aligned}
$$

The first clause says that the empty set is a member of any power-set. The second rule says that a set obtained from a subset $L$ T of $S$ by adding an element $Z \in S-L$ is a subset of $S$ and thus is also a member of the power-set of $S$.

\subsubsection{Querying Database Schema}

The higher-order syntax of F-logic makes it possible to query and manipulate certain meta-information about the database 5 such as its schema.

Schema querying is the subject of this section. This issue was also discussed in [55] and recently in [30]. However Tthe treatment in [55] is not as general and integrated as in F-logic Twhile [30] is a relational rather than an object-oriented language. 
HoweverTthis gain in efficiency has a price. For instanceTin LDLTcomparing sets for equality is easy because LDL treats each set as an entity andTin particularT the equality operator is applicable to sets. In contrast TF-logic does not have a built-in equality operator for sets. NeverthelessTcomparing sets is possible by defining set-equality and set-containment predicates via logical rules.

To see how $\Gamma$ suppose we want to verify that the result returned by one set-valued attribute stands

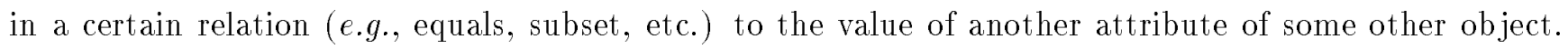
Defining these relationships in F-logic is akin to comparing relations in classical logic programming:

$$
\begin{array}{rll}
\text { notsubset }\left(O b j, A t t r, O b j^{\prime}, A t t r^{\prime}\right) & \leftarrow \text { Obj[Attr } \rightarrow X] \wedge \neg O b j^{\prime}\left[A t t r^{\prime} \rightarrow X\right] \\
\text { subset }\left(O b j, A t t r, O b j^{\prime}, A t t r^{\prime}\right) & \longleftarrow & \neg \text { notsubset }\left(O b j, A t t r, O b j^{\prime}, A t t r^{\prime}\right) \\
\text { unequal }\left(\left(O b j, A t t r, O b j^{\prime}, A t t r^{\prime}\right)\right. & \longleftarrow & \text { notsubset }\left(O b j, A t t r, O b j^{\prime}, A t t r^{\prime}\right) \\
\text { unequal }\left(\left(O b j, A t t r, O b j^{\prime}, A t t r^{\prime}\right)\right. & \leftarrow & \text { notsubset }\left(O b j^{\prime}, A t t r^{\prime}, O b j, A t t r\right) \\
\text { equal }\left(O b j, A t t r, O b j^{\prime}, A t t r^{\prime}\right) & \leftarrow & \neg \text { unequal }\left(\left(O b j, A t t r, O b j^{\prime}, A t t r^{\prime}\right)\right.
\end{array}
$$

It should be noted ThoweverTthat although expressing set-equality in F-logic is more cumbersome than in LDLTimplementing the equality operator of LDL involves the use of negation as failure Ttoo. Therefore comparing sets in F-logic and LDL has the same complexity.

Data restructuring. The next example is an adaptation from [3]. The issue here is the representation of data functions of COL [2] and grouping of LDL [16].

Consider a relation $\Gamma \operatorname{graph}(X, Y)$ Twhose tuples represent edges of a graph. The task is to re-structure this graph by representing it as a set of nodesTsuch that each node points to a set of its descendants. The corresponding F-program is very simple:

$$
\text { rebuiltGraph }[\text { nodes } \rightarrow \text { Node }[\text { descendants } \rightarrow D]] \leftarrow \operatorname{graph}(\text { Node }, D)
$$

where rebuiltGraph is a new object. This rule also shows one more way to do nesting. This timeIthough $\Gamma$ we are nesting a relation $\Gamma$ graph Trather than a class.

The reader familiar with HiLog [30] may note that this rule can be made more general if we extend the syntax of F-logic to include variables that range over predicate names:

$$
\text { rebuilt }(\text { Rel })[\text { nodes } \rightarrow \text { Node }[\text { descendants } \rightarrow D]] \leftarrow \operatorname{Rel}(\text { Node }, D) \wedge \text { Rel }: \text { relation }
$$

where Rel is a variable and relation is an appropriately defined class of binary relations. This rule will restructure any binary relation that is passed as a parameter. For instance $\Gamma$

$$
?-\text { rebuilt }(\text { yourFavourite Rel })[\text { nodes } \rightarrow \text { Node }[\text { descendants } \rightarrow D]]
$$

will return a rebuilt version of yourFavouriteGraph.

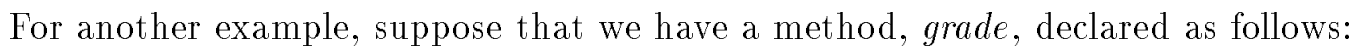

$$
\text { course[grade @student } \Rightarrow \text { integer }]
$$

A method like this would be appropriate for the use by an administrator. However Tstudents are likely to be denied access to this method in class course because this will expose grades of other students. On 


\subsubsection{Set Manipulation}

The ability to manipulate sets with ease is an important litmus test for an object-oriented data language. This subsection illustrates set-manipulation by expressing a number of popular set-manipulation opera-

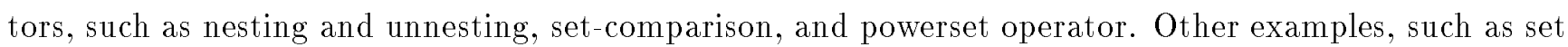
intersection and union operators can be found in [52].

Nesting and unnesting. Among the many set-related operationsT the ability to restructure objects by nesting and unnesting are among the most important ones. Specifying these operations in F-logic is quite easy. Consider a class of objects with the following structure:

$$
c\left[a t r_{1} \Rightarrow r_{1} ; a t t r_{2} \Rightarrow r_{2}\right]
$$

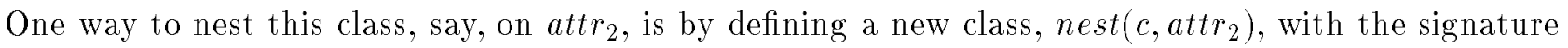

$$
\operatorname{nest}\left(c, a t t r_{2}\right)\left[a t t r_{1} \Rightarrow r_{1} ; a t t r_{2} \Rightarrow r_{2}\right]
$$

This class is populated according to the following rule:

$$
\operatorname{nested}(Y): \operatorname{nest}\left(c, a t t r_{2}\right)\left[a t t r_{1} \rightarrow Y ; \operatorname{attr}_{2} \rightarrow Z\right] \leftarrow X: c\left[a t t r_{1} \rightarrow Y ; \operatorname{attr}_{2} \rightarrow Z\right]
$$

It is easy to see from either the semantics or the proof theory that this rule has the following meaning: to nest $c$ on attr $_{2} \Gamma$ populate the class nest $\left(c, a t t r_{2}\right)$ with the objects of the form nested $(y)$ Tsuch that their attribute attr $_{2}$ groups all $z$ 's that occur with $y$ in some object $x$ in $c$.

Similarly consider a class with the following signature:

$$
c\left[a t t r_{1} \Rightarrow r_{1} ; a t t r_{2} \Rightarrow r_{2}\right]
$$

To unnest this class on the attribute att $_{2} \Gamma$ we define a new class $\Gamma$ unnest $\left(c, a_{t t}\right) \Gamma$ with the following signature:

$$
\text { unnest }\left(c, a t t r_{2}\right)\left[a t t r_{1} \Rightarrow r_{1} ; a t t r_{2} \Rightarrow r_{2}\right]
$$

Identities of objects in this class depend on both the source-objects (from class $c$ ) and on the values returned by attr 2 :

$$
\text { unnested }(X, Z): \text { unnest }\left(c, a t t r_{2}\right)\left[a t t r_{1} \rightarrow Y ; \operatorname{attr}_{2} \rightarrow Z\right] \leftarrow X: c\left[a t t r_{1} \rightarrow Y ; \text { attr }_{2} \rightarrow Z\right]
$$

This rule says that to unnest $c$ on attr 2 Twe must define new objects of the form unnested $(x, z) \Gamma$ for each $c$-object $\Gamma x \Gamma$ and for each value $z$ of attr $_{2}$ on $x$. In the unnested objectsTboth attributes $\operatorname{lattr}_{1}$ and attr $_{2} \Gamma$ are scalar and are defined so as to flatten the structure of the original objects in class $c$.

Set comparison. Grouping is not only easy to express in F-logic but it is also computationally more

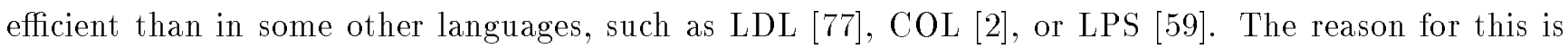
that in these languages grouping is a second-order operation that requires stratification. We refer the reader to [52] for a more complete discussion. 


\subsection{Examples of Type Declarations}

Typing is a popular concept in programming languages; in its primitive form it is used in traditional database systems under the disguise of schema declaration. In programming Ttyping can be very useful both as a debugging aid and as a means of maintaining data integrity. It allows the user to define correct usage of objects and then let the system detect ill-typed data and queries. The purpose of type declarations is to impose type-constraints on arguments of methods as well as on the results returned by the methods. For instanceГin

$$
\begin{aligned}
& \text { empl }[\text { salary } @ \text { year } \Rightarrow \text { integer }] \\
& \text { person }[\text { birthdate } \Rightarrow \text { year }]
\end{aligned}
$$

the first molecule states that salary is a function that for any empl-object would return an object in the class int Tif invoked with an argument of class year. The second clause says that birthdate is an attribute that returns a year for any person-object. Section 12 discusses how typing constraints are imposed in F-logic. In the present section we shall only give some examples of type definitionsTleaving the semantic considerations till later.

In the previous subsection Twe have seen an examples of a parametric family of classesTsuch as list $(T)$. A signature for this family can be given as follows:

$$
\operatorname{list}(T)[\text { first } \Rightarrow T ; \text { rest } \Rightarrow \operatorname{list}(T) ; \text { length } \Rightarrow \operatorname{int} ; \text { append } @ \operatorname{list}(T) \Rightarrow \operatorname{list}(T)]
$$

These signatures are parametric; they declare the attributes first Trest length Tand the method append as polymorphic functions that can take arguments of different type. As with any clause in a logic program I variables in (11) are universally quantified.

For instanceTthe signature for append says that if it is invoked on a list $($ int $)$-object with an argument

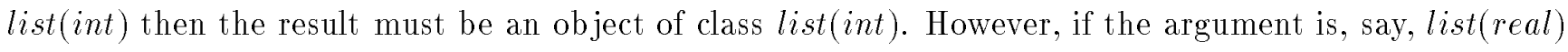
then the output must be an object of class list(real) Isince int :: real. This is because this invocations of append must conform to the signature list(real)[append @list(real) $\Rightarrow$ list(real)] Twhich is an instance of (11). Note that the output of this invocation does not have to be in class list(int) Tbecause the signatureinstance list $($ int $)[$ append $@ l i s t($ int $) \Rightarrow$ list $($ int $)]$ does not cover the invocation in question (here append is invoked on a list of realsTnot integers).

\subsection{Examples of Object Bases}

This section illustrates the expressive power of F-logic on a number of interesting and non-trivial examples $\Gamma$ which include manipulation of set and database schema Tanalogical reasoning In many casesTwe shall omit signatures because they are simple and do not illustrate new ideas (except for the list processing methods whose typing has already been discussed). Also since inheritance of properties will be discussed separately $\Gamma$ all data expressions used in the examples $\Gamma$ below $\Gamma$ are of noninheritable variety. 
In many applications $\Gamma$ the IS-A hierarchy may depend on other data $\mathrm{W}$ wich commonly happens in view definitions $\Gamma$ such as derived classes. For instance $\Gamma$

$$
\text { Car }: \text { dieselCars }(\text { Year }) \leftarrow \text { Car }: \text { car }[\text { engineType } \rightarrow \text { "diesel"; makeYear } \rightarrow \text { Year }]
$$

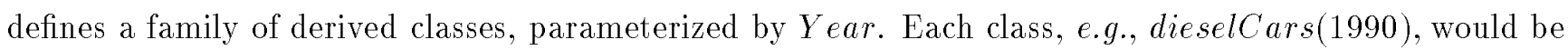
inhabited by objects that represent diesel cars made in 1990 .

FurthermoreTsometimes it is desirable to be able to create derived classes whose population is determined by the objects' structure rather than by their properties $\mathrm{Tas}$ it was in the previous example. For instance $\mathrm{Tthe}$ rule

$$
X: \text { merchandise } \longleftarrow X[\text { price } \Rightarrow()]
$$

defines a derived class $\Gamma$ merchandise $\Gamma$ that consists of all objects to which the attribute price applies. Note that an object would fall into the class merchandise if it has the attribute price Teven if the price for that merchandise has not been set yet.

Our last example concerns the set-theoretic and the lattice-theoretic operators on the class hierarchy. F-logic does not require classes to form a lattice $i$ i.e. Ta pair of classes $\Gamma$ and $c^{\prime}$ Tdoes not have to have the lowest superclass or the greatest subclass. It is not even required that $c$ and $c^{\prime}$ will have an intersection (or a union) classTi.e. Ta class whose extension (set of members) is in an intersection (resp. Tunion) of the extensions of $c$ and $c^{\prime}$. However $\Gamma$ we can define class constructors to accomplish these tasks. For instance the following rules define and $(X, Y)$ and $\operatorname{or}(X, Y)$ to be intersection and union classes of its argumentsI $X$ and $Y$ :

$$
\begin{array}{lll}
I: \operatorname{or}(X, Y) & \longleftarrow & I: X \\
I: \operatorname{or}(X, Y) & \longleftarrow & I: Y \\
I: \operatorname{and}(X, Y) & \longleftarrow & I: X \wedge I: Y
\end{array}
$$

Note that Tin the canonical model $\operatorname{cond}(X, Y)$ is not a subclass of $X$ and $Y$ Tand neither is or $(X, Y)$ their superclass. The above rules relate only extensions of the classes involved not the classes themselves. If we also wanted to relate the classes Twe would write:

$$
\begin{aligned}
& l \sup (X, Y):: C \quad \leftarrow \quad X:: C \wedge Y:: C \\
& X:: \operatorname{lsup}(X, Y) \\
& Y:: \operatorname{lsup}(X, Y) \\
& C:: \operatorname{gsub}(X, Y) \quad \leftarrow \quad C:: X \wedge C:: Y \\
& g \operatorname{sub}(X, Y):: X \\
& g \operatorname{sub}(X, Y):: Y
\end{aligned}
$$

Here lsup is a constructor that defines the lowest superclass of $X$ and $Y \Gamma$ and $g s u b$ defines greatest subclasses. Note that $\Gamma$ for instance $\operatorname{gsub}(X, Y)$ is not an intersection of the extensions of the classes $X$ and $Y$ : the extension of $g \operatorname{sub}(X, Y)$ is a subset of the extensions of $X$ and $Y \Gamma$ but the latter may have common members that are not members of $g s u b(X, Y)$. In other words $\Gamma g s u b$ and lsup construct lower and upper bounds in the class hierarchy but not in the hierarchy of class extensions. However $\Gamma$ by combining the above rules for and and gsub (and for or and lsup) it is easy to define constructors of lower and upper bounds in both hierarchies. 
Informally Tan object-base definition specifies what each method is supposed to do. Object definitions

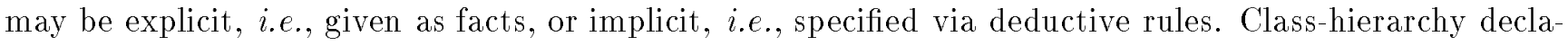
rations Tas their name suggestsTorganize objects and classes into IS-A hierarchies. Signature declarations specify the types of the arguments for each method and the type of the output they produce.

\subsection{Examples of IS-A Hierarchies}

Given an F-program and its canonic H-model Tthe IS-A hierarchy defined by the program is the set of all is-a atoms satisfied by the canonic model.

One simple example of a class-hierarchy declaration is given in Figures 2 and 3 of Section 3 . In this hierarchyए john is a student and an employee at the same time; phil is an employee but not a student. The latter is not stated explicitly and cannot be derived using the normal logical implication $\Gamma$ " $=$ " Tof Section 5. However $\ulcorner\neg$ phil $:$ student holds in the minimal model of the program of Section 3 . Since the semantics is determined by this model $\neg$ phil : student is considered as a valid statement about the class hierarchy.

The idea of class hierarchies is an important ingredient in the phenomenon known as inclusion polymorphism. For instanceTstating that students and employees are persons implies that all properties (i.e.I methods) applicable to persons must automaticly be applicable to employees and students. In Figure 4 of Section $3 \Gamma$ such properties are name (a scalar 0 -ary method that returns objects of class string) I friends (a set-valued attribute that returns objects of class person) $\Gamma$ and so on. In F-logic Cinclusion polymorphism is built into the semantics. It is manifested by a property discussed in Section $7.3 \Gamma$ called structural inheritance.

In F-logic $\Gamma$ classes are objects and so they are represented by ground id-terms. This opens a way to represent parametric families of classes using non-ground id-terms $\mathrm{W}$ which gives the flexibility needed to support parametric polymorphism. For exampleГ the following pair of clauses defines a parametric polymorphic type $\operatorname{list}(T)$ :

$$
\begin{aligned}
& n i l: \operatorname{list}(T) \\
& \operatorname{cons}(X, Y): \operatorname{list}(T) \leftarrow X: T \wedge Y: \operatorname{list}(T) \\
& \operatorname{list}(T):: \operatorname{list}(S) \longleftarrow T:: S
\end{aligned}
$$

Here list $(T)$ denotes a parametric family of classes of the form

$$
\{\operatorname{list}(t) \mid \text { where } t \text { is a ground id-term }\}
$$

For instance $\Gamma$ if int denotes the class of integers then list(int) is the class of lists of integers containing

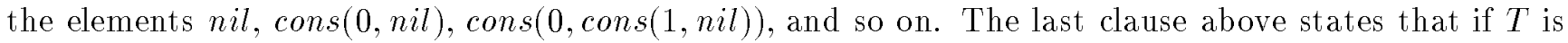
a subclass of $S$ then $\operatorname{list}(T)$ is a subclass of $\operatorname{list}(S)$. Note that this does not follow from the rest of the definition in (10) and has to be stated explicitly (if this property of lists is wanted Tof course).

The above family of list-classes will be used later to define parameterized types for list-manipulation methods. 
Then $\neg(b o b \doteq d a d(j o h n))$ must hold in all canonic models of the program. However $\Gamma$ since $b o b \doteq d a d(j o h n)$ is a logical consequence of (9)Tthis program has no equality-restricted canonic model.

\section{Queries}

A query is a statement of the form ?- $Q$ Twhere $Q$ is a molecule. ${ }^{16}$ The set of answers to ? $Q$ with respect to an $\mathrm{F}$-program $\Gamma \mathrm{P}$ Tis the smallest set of molecules that

- contains all instances $q$ of $Q$ that are found in the canonic model of $\mathbf{P}$; and

- is closed under “|=".

The first condition is obvious and does not need further comments. The second condition is needed for rather technical reasons: Suppose the database contains john[children $\rightarrow\{$ bob, sally $\}]$ and the query is $?-$ john[children $\rightarrow X]$. Then there are two instances of the query implied by this database: john[children $\rightarrow b o b]$ and john[children $\rightarrow$ sally]. However $\Gamma$ without $\mid=$-closedness $\Gamma$ $j o h n[$ children $\rightarrow\{b o b$, sally $\}]$ would not be considered an answer to the queryTeven though it is a logical consequence of the first two answers. On the other hand $\Gamma$ john[children $\rightarrow\{b o b$, sally $\}$ ] would be viewed as an answer to a logically equivalent query $?-$ john $[$ children $\rightarrow\{X, Y\}]$.

Closure with respect to " $=$ " eliminates this anomaly. It also makes it easier to talk about query equivalence and containment without getting bogged down in minor syntactic differences that may occur in essentially similar queries.

\section{The Structure of F-logic Programs}

F-programs specify what each method is supposed to doTdefine method signatures Tand organize objects along class hierarchies. ThusTevery program can be split into three disjoint componentsTaccording to the type of information they specify:

- The IS-A hierarchy declaration. This part of the F-program consists of the rules whose head-literal is an is-a assertion.

- The signature declaration. This part contains rules whose head literal is an object-molecule that is built out of signature expressions only.

- The object-base definition. This part consists of rules whose head literals do not contain signatures or is-a expressionsTi.e.Trules whose heads are either predicates or object-molecules built exclusively out of data expressions.

Note that the above classification considers rule-heads only. It is legal $\Gamma$ therefore $\mathrm{Cfor}$ rules in one component to have body-literals defined in other components. In fact Tas we shall seeTcertain applications may need such flexibility.

\footnotetext{
${ }^{16}$ This does not limit generality, as every query can be reduced to this form by adding appropriate rules.
} 


\section{Canonic Models and Equality}

Equality has always been a thorny issue in logic programming because it does not easily succumb to efficient treatment. As a result $\Gamma$ most logic programming systems-and all commercial ones-have so called "freeness axioms" built into them. ${ }^{15}$ In practice $\Gamma$ this boils down to the restriction that prohibits equality predicates from appearing in the heads of program clauses.

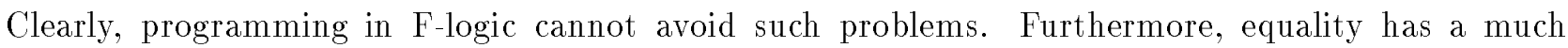
more prominent role in object-oriented programming than in classical logic programming. In F-logic $\Gamma$ for

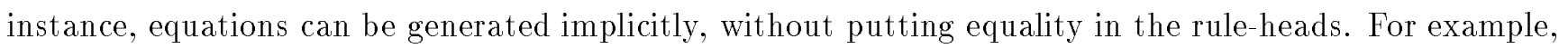
consider the following simple F-program:

$$
j o h n[\text { father } \rightarrow \text { bob }] \quad \text { john }[\text { father } \rightarrow \operatorname{dad}(j o h n)]
$$

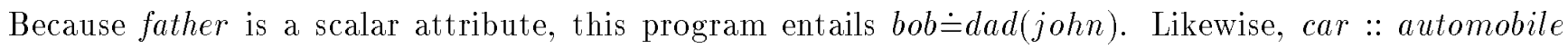
and automobile :: car together entail car $\doteq$ automobile because the class hierarchy must be acyclic.

Apart from the usual $\mathrm{C}$ computational problems associated with the equality implicit generation of equations may be problematic from the practical point of view. Indeed Tmultiply-defined scalar methodsI such as father above or cycles in the class-hierarchy may be unintentionall a result of programmer's

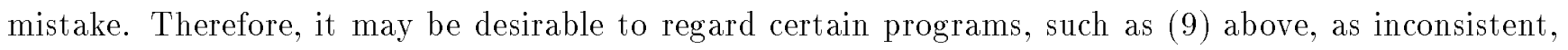
unless $d a d(j o h n) \doteq b o b$ is also defined explicitly. Of courseTsince the above program obviously has many models "inconsistency" here should be taken to mean the absence of canonic models. FurthermoreTthe notion of canonic models needs special adjustment for equality implicit equality may well have canonic models in the usual sense. For instance $(9)$ certainly has a

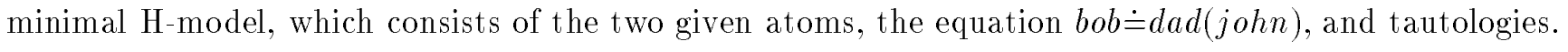

The canonic models that take special care of the equality will henceforth be called equality-restricted canonical models Which is defined next.

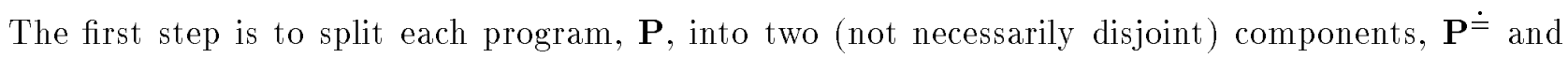
$\mathbf{P}^{\text {rest. }}$. The equality definition $\Gamma \mathbf{P}^{\doteq} \Gamma$ is the part that determines the equality theory of $\mathbf{P}$. The second component $\Gamma \mathbf{P}^{\text {rest }}$ Tdescribes the rest.

For simplicity $\Gamma$ we give a definition that relies on the assumption that the equality definition $\Gamma \mathbf{P}^{\doteq} \Gamma h a s$ only one canonic H-model. HoweverTit does not assume any specific theory of canonic models. Let $\mathrm{L}^{\doteq}$ be the (usual) canonical $\mathrm{H}$-model of $\mathbf{P}^{\doteq}$ and let $E Q$ be the set of all non-trivial equations in $\mathrm{L}^{\doteq}$. (An equation $\Gamma s \doteq t \Gamma$ is non-trivial if and only if $s$ and $t$ are not identical.)

Definition 11.1 (Equality-restricted Canonical Models) Let $\mathbf{M}$ be a canonical H-model of $\mathbf{P}$ (we do not assume that $\mathbf{M}$ is unique). We say that $\mathbf{M}$ is an equality-restricted canonical model of $\mathbf{P}$ if the set of nontrivial equations in $\mathbf{M}$ coincides with $E Q$.

Coming back to our example $\Gamma$ suppose that $b o b \doteq d a d(j o h n)$ is not in the equality-defining part of (9).

\footnotetext{
${ }^{15}$ In logic programming, freeness axioms are also known under the name of Clark's Equality Theory.
} 


\subsection{Logic Programs and their Semantics}

Perhaps the most popular classes of logic programs is the class of Horn programs. A Horn F-program consists of Horn rules Which are clauses of the form

$$
\text { head } \leftarrow \text { body }
$$

where head is an F-molecule and body is a conjunction of F-molecules. Since (8) is a clause Tthis implies that all its variables are implicitly universally quantified.

Just as in classical theory of logic programs intersection property. Thus $\Gamma$ the intersection of all H-models of a Horn program $\Gamma \mathrm{P} \Gamma$ is also an $\mathrm{H}$-model of $\mathbf{P}$. This model is also the least $\mathrm{H}$-model of $\mathbf{P}$ with respect to set-inclusion (recall that $\mathrm{H}$-structures are sets of molecules).

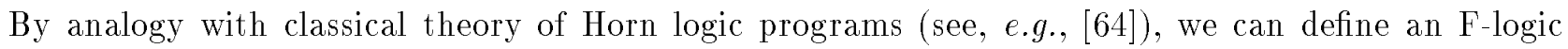
counterpart of the well-known $T_{\mathbf{P}}$ operator that $\Gamma$ given a Horn F-program $\mathbf{P}$ T maps H-structures of $\mathbf{P}$ to other H-structures of $\mathbf{P}$. Given an H-structureГIГ $T_{\mathbf{P}}(\mathbf{I})$ is defined as the smallest H-structure that contains the following set: ${ }^{13}$

$$
\left\{\text { head } \mid \text { head } \leftarrow l_{1} \wedge \cdots \wedge l_{n} \text { is a ground instance of a rule in } \mathbf{P} \text { and } l_{1}, \ldots, l_{n} \in \mathbf{I}\right\}
$$

Following a standard recipe $\Gamma$ it is easy to prove that the least fixpoint of $T_{\mathbf{P}}$ coincides with the least $\mathrm{H}$-model of $\mathbf{P}$ and that a ground $\mathrm{F}$-molecule $\Gamma$ Tis in the least $\mathrm{H}$-model of $\mathbf{P}$ if and only if $\mathbf{P}=\varphi$.

Although Horn programs can be used for a large number of applications their expressive power is limited. For more expressiveness $\mathrm{it}$ is necessary to relax the restrictions on the form of the rules and (referring to (8) above) allow negated F-molecules in body. Such programs will be called generalized (or normal) F-programs.

For generalized programs $\Gamma$ the elegant connection between fixpoints $\Gamma$ minimal models $\Gamma$ and logical entailment holds no more. In fact $\Gamma$ such programs may have several minimal models and the "right" choice is not always obvious. HoweverTit is generally accepted that the semantics of a generalized logic program is given by the set of its canonic models which is a subset of the set of all models of the program. AlasTin many cases there is no agreement as to which models deserve to be called canonic. Nevertheless $\Gamma$ for a vast class of programsTcalled locally stratified programsTsuch an agreement had been reachedTand it was shown in [81] that every such program has a unique canonic H-model. ${ }^{14}$ For a locally stratified program its unique canonic model goes under the name perfect model.

In Appendix ATwe propose a perfect-model semantics for locally stratified F-programsTwhich is an adaptation from [81]. For our current needs Thowever Twe shall assume that some canonic H-model exists for each F-program under consideration; details of these models will be immaterial for the discussions that follow. MoreoverTsince most of our examples are based on Horn programs $\Gamma$ the canonic models of these programs coincide with their unique minimal models.

\footnotetext{
${ }^{13} \mathrm{By}$ itself, this set may not be an H-structure because of the closure properties that an H-structure must satisfy (see Section 8).

${ }^{14}$ We consider only definite rules, i.e., rules that do not have disjunctions in the head.
} 
denote the derivation sequence that refutes $\mathbf{T}_{2}=S^{\prime} \cup\{L\}$ (which exists by the inductive assumption). Since $\mathbf{S}^{\prime} \subset \mathbf{S}$ and $\mathbf{S} \vdash L$ Tit follows that if we apply dedseq $q_{1}$ to $\mathbf{S}$ and then follow this up with steps from dedseq $q_{2}$ Twe shall refute $\mathbf{S}$.

Proposition 10.6 There exists a unification algorithm that, given a pair of molecules $T_{1}$ and $T_{2}$, yields a complete set of mgu's of $T_{1}$ into $T_{2}$.

Proof: The algorithm is given in Appendix BГand its correctness is proved in Lemma B.1.

Lemma 10.7 (Lifting Lemma) Suppose $C_{1}, C_{2}$ are clauses and $C_{1}^{\prime}, C_{2}^{\prime}$ are their instances, respectively. If $D^{\prime}$ is derived from $C_{1}^{\prime}$ and $C_{2}^{\prime}$ (or from $C_{1}^{\prime}$ alone) using one of the derivation rules, then there exists a clause, $D$, such that

- $D$ is derivable from the factors of $C_{1}$ and $C_{2}$ (resp., from $C_{1}$ alone) via a single derivation step;

- This derivation step uses the same inference rule as the one that derived $D^{\prime}$; and

- $D^{\prime}$ is an instance of $D$.

Proof: Consider each derivation rule separately. The proof in each case is similar to that in predicate calculus since the notion of substitution is the same in both logics. Simple (but tedious) details are left as an exercise.

Theorem 10.8 (Completeness of F-logic Inference System) If a set $\mathrm{S}$ of clauses is unsatisfiable, then there is a refutation of $\mathbf{S}$.

Proof: The proof is standard. Consider $\mathbf{S}^{*}$ Tthe set of all ground instances of $\mathbf{S}$. By the ground case (Theorem 10.5) Tthere is a refutation of $\mathbf{S}^{*}$. With the help of Lifting LemmaTthis refutation can be then lifted to a refutation of $\mathbf{S}$.

\section{Data Modeling in F-logic}

In this section $\Gamma$ we define the notions of a logic program $\Gamma$ a database $\Gamma$ and a query $\Gamma$ and then illustrate the use of F-logic on a number of simpleTyet non-trivial examples. We shall use the terms "deductive database" (or simply a database) and "logic program" interchangeably. As a first cutTwe could say that a logic program in F-logic (abbr. Tan F-program) is an arbitrary set of F-formulae. HoweverTas in classical logic programming $\Gamma$ this definition is way too general and both pragmatic and semantic considerations call for various restrictions on the form of the allowed formulas. 
(i) $P$ is an is-a assertion or a predicate:

If $P$ is an is-a assertionTitem (2) in the construction of $\mathbf{M}$ in Section 8 can be used to show that $\mathbf{M}=P$ if and only if $P \in \mathbf{D}(\mathbf{S})$. If $P$ is a predicateTitem (9) can be used to show the same.

(ii) $P$ is an object molecule composed of atoms $\tau_{1}, \ldots, \tau_{n}$ :

Then $\mathbf{M} \models P$ if and only if $\mathbf{M} \models \tau_{i} \Gamma i=1, \ldots, n$. By items $(5) \Gamma(6) \Gamma(7) \Gamma$ or $(8)$ of Section 8 (depending on whether the method expression in $\tau_{i}$ is scalar or set-valued and whether it is a data or a signature expression) $\Gamma$ it follows that $\mathbf{M} \models \tau_{i}$ if and only if $\tau_{i} \in \mathbf{D}(\mathbf{S})$. Therefore by the definition of $\mathbf{D}(\mathbf{S})$ T there are molecules $Q_{1}, \ldots, Q_{n}$ deducible from $\mathbf{S} \Gamma$ such that $\tau_{i}$ is a submolecule of $Q_{i}$ Tfor $i=1, \ldots, n$. Let $Q$ be the canonical merge of $Q_{1}, \ldots, Q_{n}$. Then $P$ is a submolecule of $Q$ (since every constituent atom of $P$ is also a constituent atom of $Q$ ) and $Q$ is deducible from $\mathbf{S}$ ( since $Q_{1}, \ldots, Q_{n}$ are deducible from $\mathbf{S}$ and $Q$ is obtained from $Q_{1}, \ldots, Q_{n}$ by the merging rule). Hence $P$ is in $\mathbf{D}(\mathbf{S})$ Twhich proves (7).

By the definition of $\mathbf{D}(\mathbf{S})$ Iif $P \in \mathbf{S}$ is a positive literal then $P \in \mathbf{D}(\mathbf{S})$. Hence $\Gamma$ by $(7) \Gamma \mathbf{M} \models P$. For every negative literal $\neg P$ in $\mathbf{S} \Gamma P$ is not a submolecule of any molecule deducible from $\mathbf{S} \Gamma$ by the assumption made at the beginning of the proof. So $P$ is not in $\mathbf{D}(\mathbf{S})$. Again $\Gamma$ by $(7) \Gamma \mathbf{M} \forall P$ and therefore $\mathbf{M} \models \neg P$. Thus $\Gamma \mathrm{M}$ satisfies every literal of $\mathbf{S}$ Tthat is $\Gamma$ it is a model for $\mathbf{S}$.

Theorem 10.5 (Completeness of ground deduction) If a set of ground clauses, $\mathrm{S}$, is unsatisfiable then there exists a refutation of $\mathbf{S}$.

Proof: By Herbrand's Theorem $\Gamma$ we may assume that $\mathbf{S}$ is finite. Suppose $\mathbf{S}$ is unsatisfiable. We will show that there is a refutation of $\mathbf{S}$ using a technique due to Anderson and Bledsoe [8]. The proof is carried out by induction on the parameter excess $(\mathbf{S})$ Tthe number of "excess literals" in $\mathbf{S}$ :

$\operatorname{excess}(\mathbf{S}) \stackrel{\text { def }}{=}($ the number of occurrences of literals in $\mathbf{S})-($ the number of clauses in $\mathbf{S})$.

Basis: excess $(\mathbf{S})=0$. In this caseTthe number of clauses in $\mathbf{S}$ equals the number of occurrences of literals in $\mathbf{S}$. Hence either $\square \in \mathbf{S}$ and we are doneTor every clause in $\mathbf{S}$ is a literal. In the latter case $\Gamma$ by Lemma 10.4ГS $\neg \neg$ and $\mathbf{S} \vdash Q$ for some molecules $P \Gamma Q$ such that $P \sqsubseteq Q$. Applying the resolution rule to $\neg P$ and $Q \Gamma$ we obtain the empty clause.

Induction Step: excess $(\mathbf{S})=n>0$. In this case $\Gamma$ there must be a clause $C$ in $\mathbf{S}$ that contains more than one literal. Let us separate this clause from the other clauses and write $\mathbf{S}=\{C\} \cup \mathbf{S}^{\prime} \Gamma$ where $C=L \vee C^{\prime} \quad\left(C^{\prime} \neq \square\right.$ since we have assumed that $C$ contains more than one literal $)$. By the distributivity law $\Gamma\left\{L \vee C^{\prime}\right\} \cup \mathbf{S}^{\prime}$ is unsatisfiable if and only if so are $\mathbf{T}_{1}=\left\{C^{\prime}\right\} \cup \mathbf{S}^{\prime}$ and $\mathbf{T}_{2}=\{L\} \cup \mathbf{S}^{\prime}$. Since $\operatorname{excess}\left(\mathbf{T}_{1}\right)<n$ and excess $\left(\mathbf{T}_{2}\right)<n$ Tthe induction hypothesis ensures that there are refutations of $\mathbf{T}_{1}$ and $\mathbf{T}_{2}$ separately. Therefore $\Gamma \mathbf{T}_{1} \vdash \square \Gamma$ where $\square$ is the empty clause. Let dedseq denote the deduction sequence that derives $\square$ from $\mathbf{T}_{1}$. Applying the deductive steps in dedseq $q_{1}$ to $\mathbf{S}$ Twe can derive either $L$ or

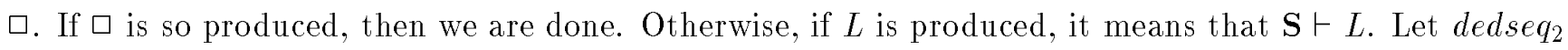




\subsection{A Sample Proof}

Consider the following set of clauses:
i. $\quad a:: b$
iv. $\quad r[a t t r \rightarrow a]$
ii. $\quad p(a)$
v. $\quad r[a t t r \rightarrow f(S)] \vee \neg p(X) \vee \neg O[M @ X \Rightarrow S]$
iii. $\quad c[m @ b \Rightarrow(v, w)]$
vi. $\quad \neg p(f(Z))$

We can refute the above set using the following sequence of derivation steps 5 where $\theta$ denotes the unifier used in the corresponding step:
vii. $\quad r[a t t r \rightarrow f(S)] \vee \neg O[M @ a \Rightarrow S]$
by resolving (ii) and (v); $\theta=\{X \backslash a\}$
viii. $\quad c[m @ a \Rightarrow(v, w)]$
by input-type restriction from (i) and (iii)
ix. $\quad r[a t t r \rightarrow f(v)]$
x. $\quad a \doteq f(v)$
by resolving (viii) with (vii); $\theta=\{O \backslash c, S \backslash v, M \backslash m\}$
xi. $\quad p(f(v))$
by the rule of scalarityГusing (iv) and (ix)
xii.
by paramodulation $\Gamma u \operatorname{sing}($ ii) and $(\mathrm{x})$
by resolving (vi) with (xi); $\theta=\{Z \backslash v\}$

All steps in this derivation are self-explanatory. We would like to point out $\mathrm{th}$ though $\mathrm{That} \theta$ in Step (ix) is an asymmetric unifier that unifies the atom $O[M @ a \Rightarrow S]$ into the molecule (iii).

\subsection{Completeness of the Proof Theory}

We follow the standard strategy for proving completeness Tadapted from classical logic. First ГHerbrand's Theorem is used to establish completeness for the ground case. Then $\Gamma$ an analogue of Lifting Lemma shows that ground refutations can be "lifted" to the nonground case.

Lemma 10.4 Let $\mathrm{S}$ be a set of ground F-literals. If $\mathrm{S}$ is unsatisfiable then there are molecules $P$ and $Q$ such that $P \sqsubseteq Q, \neg P \in \mathrm{S}$ and $\mathrm{S} \vdash Q$. (When $P, Q$ are P-molecules or is-a assertions, $P \sqsubseteq Q$ should be taken to mean that $P$ is identical to $Q$.)

Proof: Suppose to the contraryT that there are no molecules $P$ and $Q \Gamma$ such that $P \sqsubseteq Q \Gamma \neg P \in \mathrm{S} \Gamma$ and $\mathbf{S} \vdash Q$. We will show that then $\mathbf{S}$ must be satisfiable. Consider a set of molecules $\Gamma$

$$
\mathbf{D}(\mathbf{S}) \stackrel{\text { def }}{=}\{P \mid P \text { is a submolecule of some molecule } Q \text { such that } \mathbf{S} \vdash Q\} .
$$

Section 8 shows that every H-structureГHГhas a corresponding F-structure $\mathbf{I}_{H}$ such that $\mathbf{H}=\mathbf{S}$ if and only if $\mathbf{I}_{H}=\mathbf{S}$. Applying the same construction to $\mathbf{D}(\mathbf{S})$ we obtain an F-structure $\mathbf{M}$. Since $\mathbf{D}(\mathbf{S})$ is closed under deduction $\Gamma i t$ is easy to verify that $\mathbf{M}$ is molecule $P$ :

$$
\mathbf{M} \models P \quad \text { if and only if } P \in \mathbf{D}(\mathbf{S})
$$

The "if"-direction follows from soundness of the derivation rules. For the "only if"-direction $\Gamma$ consider the following two cases: 


\begin{tabular}{|c|c|}
\hline \multirow[t]{2}{*}{ Scalarity: } & $\begin{array}{c}P\left[M t h d @ Q_{1}, \ldots, Q_{k} \rightarrow R\right] \vee C_{1}, P^{\prime}\left[M t h d^{\prime} @ Q_{1}^{\prime}, \ldots, Q_{k}^{\prime} \rightarrow R^{\prime}\right] \vee C_{2} \\
\theta=m g u\left(\left\langle P, M t h d, Q_{1}, \ldots, Q_{k}\right\rangle,\left\langle P^{\prime}, M t h d^{\prime}, Q_{1}^{\prime}, \ldots, Q_{k}^{\prime}\right\rangle\right)\end{array}$ \\
\hline & $\begin{array}{l}\qquad \theta\left(R \doteq R^{\prime} \vee C_{1} \vee C_{2}\right) \\
\text { Similarly for inheritable scalar expressions } \Gamma \text { where } \rightarrow \text { is replaced with } \bullet\end{array}$ \\
\hline Merging: & $\frac{P[\ldots] \vee C, P^{\prime}[\ldots] \vee C^{\prime}, \theta=m g u\left(P, P^{\prime}\right), \quad L^{\prime \prime}=\operatorname{merge}\left(\theta(P[\ldots]), \theta\left(P^{\prime}[\ldots]\right)\right)}{L^{\prime \prime} \vee \theta\left(C \vee C^{\prime}\right)}$ \\
\hline Elimination: & $\frac{\neg P[] \vee C}{C}$ \\
\hline
\end{tabular}

Figure 8: Summary of the Miscellaneous Inference Rules

\subsection{Remarks}

With such multitude of inference rules $\mathrm{a}$ a natural concern is whether there might be an efficient evaluation procedure for F-logic queries. The answer to this question is positive: F-logic queries can be evaluatedI sayCbottom-up and the optimization strategies developed for deductive databases (e.g. TMagic Sets and Templates [18Г83]) are applicable here as well.

Another important point is that one does not need to use some of the inference rules at run time. For instance $i n$ proof-theoretic terms The purpose of static type checking is to obviate the need in using the typing rules at run time. Likewise $\mathrm{T}$ a compile-time acyclicity-checking algorithm could be used to get rid of the IS-A acyclicity rule at run time. A practical system is also likely to limit the use of the rule of scalarity. For instance $\mathrm{T}$ this rule may be used to generate run-time warnings regarding possible inconsistencies detected in scalar methodsTbut not to do inference.

\subsection{Soundness of the Proof Theory}

Given a set $\mathbf{S}$ of clausesTa deduction of a clause $C$ from $\mathbf{S}$ is a finite sequence of clauses $D_{1}, \ldots, D_{n}$ such that $D_{n}=C$ and $\Gamma$ for $1 \leq k \leq n \Gamma D_{k}$ is either

- a member of $\mathbf{S} \Gamma$ or

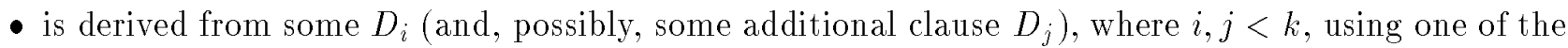
core $i s-a \Gamma t y p e$ or miscellaneous inference rules.

A deduction ending with the empty clause $\square \square$ is called a refutation of $\mathbf{S}$. If $C$ is deducible from $\mathbf{S} \Gamma$ we shall write $\mathbf{S} \vdash C$.

Theorem 10.3 (Soundness of F-logic Deduction) If $\mathrm{S} \vdash C$ then $\mathrm{S} \mid=C$.

Proof: Directly follows from the closure properties given in Section 7 and from the form of the inference rules. 
A similar rule exists for inheritable scalar expressions. The only difference is that $\rightarrow$ is replaced with $\bullet$ in $W$ and $W^{\prime}$.

Another miscellaneous rule is called merging; it seeks to combine information contained in different object molecules. Let $L_{1}$ and $L_{2}$ be a pair of such molecules with the same object id. An object-molecule $L$ is called a merge of $L_{1}$ and $L_{2}$ Tif the set of constituent atoms of $L$ is precisely the union of the sets of constituent atoms of $L_{1}$ and $L_{2}$. A pair of molecules can be merged in several different ways when they have common set-valued methods. For exampleTthe terms

$$
\begin{aligned}
& T[\operatorname{Scal} M \rightarrow d ; \text { Set } M \rightarrow e ; \operatorname{Set} M @ X \rightarrow b] \\
& T[\operatorname{Scal} M \rightarrow g ; \text { Set } M @ Y \rightarrow h ; \operatorname{Set} M @ X \rightarrow c]
\end{aligned}
$$

have more than one merge:

$$
T[S \operatorname{cal} M \rightarrow d ; \text { Scal } M \rightarrow g ; \text { Set } M \rightarrow e ; \text { Set } M @ Y \rightarrow h ; \text { Set } M @ X \rightarrow b ; \text { Set } M @ X \rightarrow c]
$$

and

$$
T[S \text { cal } M \rightarrow d ; \text { ScalM } \rightarrow g ; \text { Set } M \longrightarrow e ; \operatorname{Set} M @ Y \rightarrow h ; \text { Set } M @ X \rightarrow\{b, c\}]
$$

However $\Gamma$ we distinguish certain kind of merges that do have the uniqueness property. We call them canonical merges.

An invocation of a method consists of the method's nameTits arguments $\Gamma$ and the arrow specifying

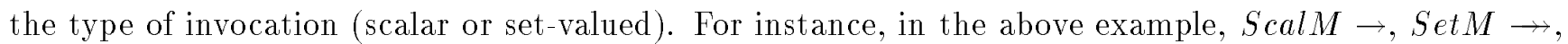
Set $M @ X \rightarrow \Gamma$ and $S e t M @ Y \rightarrow$ are all distinct invocations. A canonical merge of $L_{1}$ and $L_{2}$ Tdenoted merge $\left(L_{1}, L_{2}\right)$ Tis a merge that does not contain repeated identical invocations of set-valued methods. In the above $\Gamma(6)$ is a canonical merge of $(3)$ and (4). Clearly $\operatorname{merge}\left(L_{1}, L_{2}\right)$ is unique up to a permutation of atoms and id-terms in the ranges of set-valued methods.

Merging: Consider a pair of standardized apart clauses $\Gamma=L \vee C$ and $W^{\prime}=L^{\prime} \vee C^{\prime} \Gamma$ where both $L$ and $L^{\prime}$ are object molecules. Let $\theta$ be an mgu unifying the oid parts of $L$ and $L^{\prime}$. Let $L^{\prime \prime}$ denote the canonical merge of $\theta(L)$ and $\theta\left(L^{\prime}\right)$. The merging ruleTthen $\Gamma$ sanctions the following derivation:

$$
\text { from } W \text { and } W^{\prime} \text { derive } L^{\prime \prime} \vee \theta\left(C \vee C^{\prime}\right)
$$

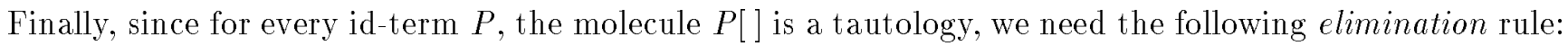

Elimination: $\quad$ If $C$ is a clause and $P$ an id-term then:

$$
\text { from } \neg P[] \vee C \text { derive } C
$$

Notice that if $C$ is an empty clause then the elimination rule would derive an empty clause as well. 
Type inheritance: $\quad \frac{P\left[M t h d @ Q_{1}, \ldots, Q_{k} \Rightarrow T\right] \vee C,\left(S^{\prime}:: P^{\prime}\right) \vee C^{\prime}, \theta=m g u\left(P, P^{\prime}\right)}{\theta\left(S^{\prime}\left[M t h d @ Q_{1}, \ldots, Q_{k} \Rightarrow T\right] \vee C \vee C^{\prime}\right)}$

Similarly for set-valued methods

Input restriction: $\frac{P\left[M t h d @ Q_{1}, \ldots, Q_{i}, \ldots, Q_{k} \Rightarrow T\right] \vee C,\left(Q_{i}^{\prime \prime}:: Q_{i}^{\prime}\right) \vee C^{\prime}, \theta=m g u\left(Q_{i}, Q_{i}^{\prime}\right)}{\theta\left(P\left[M t h d @ Q_{1}, \ldots, Q_{i}^{\prime \prime}, \ldots, Q_{k} \Rightarrow T\right] \vee C \vee C^{\prime}\right)}$

Similarly for set-valued methods

Output relaxation: $\frac{P\left[M t h d @ Q_{1}, \ldots, Q_{k} \Rightarrow R\right] \vee C,\left(R^{\prime}:: R^{\prime \prime}\right) \vee C^{\prime}, \theta=m g u\left(R, R^{\prime}\right)}{\theta\left(P\left[M t h d @ Q_{1}, \ldots, Q_{k} \Rightarrow R^{\prime \prime}\right] \vee C \vee C^{\prime}\right)}$

Similarly for set-valued methods

Figure 7: Summary of the Type-Inference Rules

Input-type restriction: $\quad$ Let $W=P\left[M\right.$ thd $\left.@ Q_{1}, \ldots, Q_{i}, \ldots, Q_{k} \Rightarrow T\right] \vee C$ and $W^{\prime}=\left(Q_{i}^{\prime \prime}:: Q_{i}^{\prime}\right) \vee C^{\prime}$ be standardized apart. Suppose also that $Q_{i}$ and $Q_{i}^{\prime}$ have an mgu $\theta$. The input restriction rule states:

from $W$ and $W^{\prime}$ derive $\theta\left(P\left[M t h d @ Q_{1}, \ldots, Q_{i}^{\prime \prime}, \ldots, Q_{k} \Rightarrow T\right] \vee C \vee C^{\prime}\right)$

Here $Q_{i}^{\prime \prime}$ replaces $Q_{i}$. A similar rule exists for set-valued methods.

Output-type relaxation: Consider clauses $W=P\left[M\right.$ thd $\left.@ Q_{1}, \ldots, Q_{k} \Rightarrow R\right] \vee C$ and $W^{\prime}=\left(R^{\prime}::\right.$ $\left.R^{\prime \prime}\right) \vee C^{\prime}$ with no common variables $\Gamma$ and suppose $R$ and $R^{\prime}$ have an mgu $\theta$. The output relaxation rule then Tstates:

from $W$ and $W^{\prime}$ derive $\theta\left(P\left[M t h d @ Q_{1}, \ldots, Q_{k} \Rightarrow R^{\prime \prime}\right] \vee C \vee C^{\prime}\right)$

Similar rules apply to set-valued methods.

We also note that in the first two inference rules above $T$ is either an id-term or ( ).

\subsection{Miscellaneous Inference Rules}

The property that scalar methods return at most one value is enforced by the following rule:

Scalarity: Consider a pair of clauses that share no common variables:

$$
W=P\left[M t h d @ Q_{1}, \ldots, Q_{k} \rightarrow R\right] \vee C \text { and } W^{\prime}=P^{\prime}\left[M t h d^{\prime} @ Q_{1}^{\prime}, \ldots, Q_{k}^{\prime} \rightarrow R^{\prime}\right] \vee C^{\prime}
$$

Suppose there is an mgu $\theta$ that unifies the tuple of id-terms $\left\langle P, M\right.$ thd $\left., Q_{1}, \ldots, Q_{k}\right\rangle$ with the tuple $\left\langle P^{\prime}, M t h d^{\prime}, Q_{1}^{\prime}, \ldots, Q_{k}^{\prime}\right\rangle$. The rule of scalarity then says:

from $W$ and $W^{\prime}$ derive $\theta\left(\left(R \doteq R^{\prime}\right) \vee C \vee C^{\prime}\right)$ 


\begin{tabular}{ll} 
IS-A Reflexivity: & $\mathrm{X}:: \mathrm{X}$ \\
IS-A acyclicity: & $\frac{(P:: Q) \vee C,\left(Q^{\prime}:: P^{\prime}\right) \vee C^{\prime}, \theta=m g u\left(\langle P, Q\rangle,\left\langle P^{\prime}, Q^{\prime}\right\rangle\right)}{\theta\left((P \doteq Q) \vee C \vee C^{\prime}\right)}$ \\
IS-A transitivity: & $\frac{(P:: Q) \vee C,\left(Q^{\prime}:: R^{\prime}\right) \vee C^{\prime}, \theta=m g u\left(Q, Q^{\prime}\right)}{\theta\left(\left(P:: R^{\prime}\right) \vee C \vee C^{\prime}\right)}$ \\
Subclass inclusion: & $\frac{(P: Q) \vee C,\left(Q^{\prime}:: R^{\prime}\right) \vee C^{\prime}, \theta=m g u\left(Q, Q^{\prime}\right)}{\theta\left(\left(P: R^{\prime}\right) \vee C \vee C^{\prime}\right)}$ \\
\hline
\end{tabular}

Figure 6: Summary of the IS-A Inference Rules

follows:

from $W$ and $W^{\prime}$ derive $\theta\left((P \doteq Q) \vee C \vee C^{\prime}\right)$

Note that IS-A reflexivity and IS-A acyclicity imply reflexivity of equality. Indeed $\Gamma$ since $X:: X$ is an

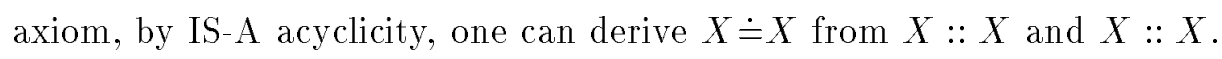

IS-A transitivity: Let $W=(P:: Q) \vee C$ and $W^{\prime}=\left(Q^{\prime}:: R^{\prime}\right) \vee C^{\prime}$ be standardized apart and let $\theta$ be an mgu of $Q$ and $Q^{\prime}$. The transitivity rule then $\Gamma$ is:

from $W$ and $W^{\prime}$ derive $\theta\left(\left(P:: R^{\prime}\right) \vee C \vee C^{\prime}\right)$

Subclass inclusion: Let $W=(P: Q) \vee C$ and $W^{\prime}=\left(Q^{\prime}:: R^{\prime}\right) \vee C^{\prime}$ be standardized apart and let $\theta$ be an mgu of $Q$ and $Q^{\prime}$. Then the subclass inclusion rule says:

from $W$ and $W^{\prime}$ derive $\theta\left(\left(P: R^{\prime}\right) \vee C \vee C^{\prime}\right)$

\subsection{Type Inference Rules}

Signature expressions have the properties of type inheritance $\Gamma$ input-type restriction $\Gamma$ and output-type relaxation that are captured by the following inference rules:

Type inheritance: $\quad$ Let $W=P\left[M t h d @ Q_{1}, \ldots, Q_{k} \Rightarrow T\right] \vee C$ and $W^{\prime}=\left(S^{\prime}:: P^{\prime}\right) \vee C^{\prime}$ be a pair of clauses with no common variables $\Gamma$ and suppose $P$ and $P^{\prime}$ have an mguT $\theta$. The type inheritance rule states the following:

from $W$ and $W^{\prime}$ derive $\theta\left(S^{\prime}\left[M t h d @ Q_{1}, \ldots, Q_{k} \Rightarrow T\right] \vee C \vee C^{\prime}\right)$

In other words $\Gamma S^{\prime}$ inherits the signature of $P^{\prime}$. A similar rule exists for set-valued methods. If $W=P\left[M\right.$ thd $\left.@ Q_{1}, \ldots, Q_{k} \Rightarrow T\right] \vee C$ and $W^{\prime}$ is as before $\Gamma$ then:

from $W$ and $W^{\prime}$ derive $\theta\left(S^{\prime}\left[M t h d @ Q_{1}, \ldots, Q_{k} \Rightarrow T\right] \vee C \vee C^{\prime}\right)$ 


$$
\begin{array}{ll}
\text { Resolution: } & \frac{\neg L \vee C, L^{\prime} \vee C^{\prime}, \theta=m g u_{\sqsubseteq}\left(L, L^{\prime}\right)}{\theta\left(C \vee C^{\prime}\right)} \\
\text { Factoring: } & \frac{L \vee L^{\prime} \vee C, \theta=m g u_{\sqsubseteq}\left(L, L^{\prime}\right)}{\theta(L \vee C)} \frac{\neg L \vee \neg L^{\prime} \vee C, \theta=m g u_{\sqsubset}\left(L, L^{\prime}\right)}{\theta\left(\neg L^{\prime} \vee C\right)} \\
\text { Paramodulation: } & \frac{L[T] \vee C,\left(T^{\prime} \doteq T^{\prime \prime}\right) \vee C^{\prime}, \theta=m g u\left(T, T^{\prime}\right)}{\theta\left(L\left[T \backslash T^{\prime \prime}\right] \vee C \vee C^{\prime}\right)}
\end{array}
$$

Figure 5: Summary of the Core Inference Rules

Let $L$ be unifiable into $L^{\prime}$ with the mgu $\theta$. The factoring rule is $\Gamma$ then $\Gamma$ as follows:

$$
\text { from } W \text { derive } \theta(L \vee C)
$$

In case of negative literals $\Gamma$ if $W=\neg L \vee \neg L^{\prime} \vee C$ and $L$ is unifiable into $L^{\prime}$ with the mgu $\theta \Gamma$ then the factoring rule is:

$$
\text { from } W \text { derive } \theta\left(\neg L^{\prime} \vee C\right)
$$

Clauses inferred by one of the two factoring rules are called factors of $W$. Note that in both inference rules $L$ must be unifiable into $L^{\prime}$. HoweverTin the first case ${ }^{2}$ it is the literal $L$ that survives $\Gamma$ while in the second rule it is $L^{\prime}$.

To account for the equality relation $\Gamma$ we need a paramodulation rule. We use the following standard convention: When there is a need to focus on a specific occurrence of an id-term $\Gamma T$ Tin an expression $\Gamma E$ (which can be a literal or an id-term) $\Gamma$ we may write $E[T]$. If one single occurrence of $T$ is replaced by $S$ T the result will be denoted by $E[T \backslash S]$.

Paramodulation: Consider a pair of clauses $\Gamma W=L[T] \vee C$ and $W^{\prime}=\left(T^{\prime} \doteq T^{\prime \prime}\right) \vee C^{\prime} \Gamma$ with no common variables. If $T$ and $T^{\prime}$ are id-terms unifiable with an mgu $\theta \Gamma$ then the paramodulation says:

$$
\text { from } W \text { and } W^{\prime} \text { derive } \theta\left(L\left[T \backslash T^{\prime \prime}\right] \vee C \vee C^{\prime}\right)
$$

\subsection{IS-A Inference Rules}

The following axiom and the rules capture the semantics of the subclass relationship and its interaction with class membership.

IS-A Reflexivity: The following is the IS-A reflexivity axiom:

$$
(\forall X) X:: X \text {. }
$$

IS-A acyclicity: Let $W=(P:: Q) \vee C$ and $W^{\prime}=\left(Q^{\prime}:: P^{\prime}\right) \vee C^{\prime}$ be clauses with no variables in common. Suppose that $\theta$ is an mgu of tuples $\langle P, Q\rangle$ and $\left\langle P^{\prime}, Q^{\prime}\right\rangle$ of id-terms. The acyclicity rule is as 
In predicate calculus the notion of a unifier works for an arbitrary number of terms to be unified. Extension of our definitions to accommodate an arbitrary number of id-terms $\Gamma$ P-moleculesГor is-a assertions is obvious. For object molecules $\Gamma$ we say that a substitution $\sigma$ is a unifier of $L_{1} \Gamma \ldots \Gamma L_{n}$ into $L$ if and only if $\sigma\left(L_{i}\right) \sqsubseteq L \Gamma$ for $i=1, \ldots, n$. Generalization of the notion of $\mathrm{mgu}$ is straightforward and is left as an exercise.

For notational convenienceTwe also define mgu's for tuples of id-terms. Tuples $\left\langle P_{1}, \ldots, P_{n}\right\rangle$ and $\left\langle Q_{1}, \ldots\right.$, $\left.Q_{n}\right\rangle$ are unifiable if and only if there is a substitution $\sigma$ such that $\sigma\left(P_{i}\right)=\sigma\left(Q_{i}\right) \Gamma i=1, \ldots, n$. This unifier is most general (written $\left.m g u\left(\left\langle P_{1}, \ldots, P_{n}\right\rangle,\left\langle Q_{1}, \ldots, Q_{n}\right\rangle\right)\right) \Gamma$ if and only if for every other unifier $\Gamma \mu$ Tof these tuples $\Gamma \mu=\gamma \circ \sigma$ for some substitution $\gamma$. It is easy to see that any mgu of $\left\langle P_{1}, \ldots, P_{n}\right\rangle$ and $\left\langle Q_{1}, \ldots, Q_{n}\right\rangle$ coincides with the mgu of $f\left(P_{1}, \ldots, P_{n}\right)$ and $f\left(Q_{1}, \ldots, Q_{n}\right)$ Twhere $f$ is an arbitrary $n$-ary function symbolI and $\Gamma$ therefore $\mathrm{is}$ unique.

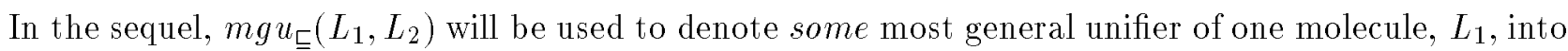
another $\Gamma L_{2}$. As shown earlierTfor object molecules $m g u_{\sqsubseteq}\left(L_{1}, L_{2}\right)$ may exist while $m g u_{\sqsubseteq}\left(L_{2}, L_{1}\right)$ may not. On the other hand $\Gamma$ unification of id-terms $\Gamma$ is-a assertions $\Gamma$ and $\mathrm{P}$-molecules is a symmetric operation. Nevertheless $\Gamma$ we can still talk about unification of one such expression into another-a convention that can often simplify the language. AlsoTfor the sake of simpler notation $\Gamma$ all our inference rules will be based on F-atoms Tnot F-molecules.

Finally $\Gamma$ we remark that $\Gamma$ as in predicate calculus $\Gamma$ prior to any application of an inference rule $\Gamma$ the clauses involved in the application must be standardized apart. This means that variables must be consistently renamed so that the resulting clauses will share none. HoweverTclauses used by an inference rule may be instances of the same clause; they can even be identical if no variables are involved.

\subsection{Core Inference Rules}

For simplicity Cbut without loss of generality Tonly binary resolution is considered. In the inherence rules $\Gamma$ below $\Gamma$ the symbols $L$ and $L^{\prime}$ will be used to denote F-literals (positive or negative) $\Gamma C$ and $C^{\prime}$ will denote clausesTand $P \Gamma Q \Gamma R \Gamma S \Gamma T$ Tetc. Twill denote id-terms.

Resolution: Let $W=\neg L \vee C$ and $W^{\prime}=L^{\prime} \vee C^{\prime}$ be a pair of clauses that are standardized apart. Let $\theta$ be an mgu of $L$ into $L^{\prime}$. The resolution rule is $\Gamma$ then $\Gamma$ as follows:

$$
\text { from } W \text { and } W^{\prime} \text { derive } \theta\left(C \vee C^{\prime}\right)
$$

Notice that when $L$ and $L^{\prime}$ are object moleculesT resolution is asymmetric since $\theta=m g u_{\sqsubseteq}\left(L, L^{\prime}\right)$ may be different from $m g u_{\sqsubseteq}\left(L^{\prime}, L\right) \Gamma$ and the latter mgu may not even exist. As in the classical case $\mathrm{Cbinary}$ resolution must be complemented with the so-called factoring rule that seeks to reduce the number of disjuncts in a clause.

Factoring: The factoring rule has two formsTdepending on the polarity of literals to be factored. For positive literals $\Gamma$ consider a clause of the form $W=L \vee L^{\prime} \vee C$ Twhere $L$ and $L^{\prime}$ are positive literals. 


\section{Unifiers}

Unification of id-termsTis-a assertionsTand P-molecules is no different than in classical logic. Let $T_{1}$ and

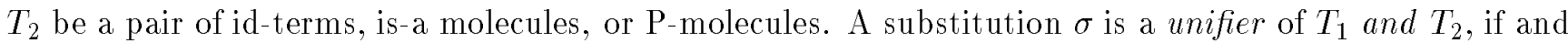
only if $\sigma\left(T_{1}\right)=\sigma\left(T_{2}\right)$. This unifier is most general $\Gamma$ written $m g u\left(T_{1}, T_{2}\right) \Gamma$ if for every unifier $\mu$ of $T_{1}$ and $T_{2}$ Tthere exists a substitution $\gamma$ Tsuch that $\mu=\gamma \circ \sigma$.

For object moleculesTrather than requiring that they must become identical after applying a unifier $\Gamma$ we ask merely that one molecule will be mapped into a submolecule of the other.

Definition 10.1 (Asymmetric Unification of Object Molecules) Let $L_{1}=S[\ldots]$ and $L_{2}=S[\ldots]$ be a pair of object molecules with the same object id $\Gamma S$. We say that $L_{1}$ is a submolecule of $L_{2} \Gamma$ denoted $L_{1} \sqsubseteq L_{2} \Gamma$ if and only if every constituent atom of $L_{1}$ (defined in Section 7 ) is also a constituent atom of $L_{2}$. A substitution $\sigma$ is a unifier of $L_{1}$ into $L_{2}$ (note the asymmetry!) if and only if $\sigma\left(L_{1}\right) \sqsubseteq \sigma\left(L_{2}\right)$.

For instance $L=S[M @ X \rightarrow V]$ is unifiable into $L^{\prime}=S[N @ Y \rightarrow W ; Z @ Y \bullet T]$ with the unifier $\{M \backslash N, X \backslash Y, V \backslash W\} \Gamma$ but not the other way around (because the atom $S[Z @ Y \bullet T]$ cannot be turned into a constituent atom of $L$ ). However $\Gamma$ a slightly different molecule $\Gamma[N @ Y \rightarrow W ; Z @ Y \rightarrow T] \Gamma$ is unifiable into $L$ with the unifier $\{N \backslash M, Y \backslash X, W \backslash V, Z \backslash M, T \backslash V\}$.

\section{Complete Sets of Most General Unifiers}

Defining most general unifiers for object molecules requires more work. Consider the terms $L_{1}=a[$ set $\rightarrow$ $\{X\}]$ and $L_{2}=a[$ set $\rightarrow\{b, c\}]$. Intuitively $\mathrm{Cthere}$ are two unifiers of $L_{1}$ into $L_{2}$ that can be called "most general:" $X \backslash b$ and $X \backslash c$. ClearlyГnone of these unifiers is more general than the other and $T$ thereforeI the definition of mgu that works for P-molecules and for is-a assertions does not work here. A common approach in such situations is to consider complete sets of most general unifiers.

Definition 10.2 (Most General Unifiers) Let $L_{1}$ and $L_{2}$ be a pair of molecules and let $\alpha \Gamma \beta$ be a pair of unifiers of $L_{1}$ into $L_{2}$. We say that $\alpha$ is more general than $\beta$ T denoted $\alpha \unlhd \beta \Gamma$ if and only if there is a substitution $\gamma$ such that $\beta=\gamma \circ \alpha$. A unifier $\alpha$ of $L_{1}$ into $L_{2}$ is most general (abbr. $\mathrm{mg}$ ) if for every unifier $\beta \Gamma \beta \unlhd \alpha$ implies $\alpha \unlhd \beta$.

A set $\Sigma$ of most general unifiers of $L_{1}$ into $L_{2}$ is complete if for every unifier $\theta$ of $L_{1}$ into $L_{2}$ there is $\alpha \in \Sigma$ such that $\alpha \unlhd \theta$.

Just as there is a unique-up-to-the-equivalence mgu in the classical caseCit easily follows from the definitions that the complete set of unifiers of $L_{1}$ into $L_{2}$ is also unique up to the equivalence. ${ }^{12}$ An algorithm that computes a complete set of mgu's appears in Appendix B.

\footnotetext{
${ }^{12}$ A set of unifiers, $\Omega_{1}$, is equivalent to $\Omega_{2}$ if for every $\sigma_{1} \in \Omega_{1}$ there is $\sigma_{2} \in \Omega_{2}$ such that $\sigma_{2} \unlhd \sigma_{1}$; and vice versa, for every $\sigma_{2} \in \Omega_{2}$ there is $\sigma_{1} \in \Omega_{1}$ such that $\sigma_{1} \unlhd \sigma_{2}$.
} 


\section{Proof Theory}

This section describes a sound and complete proof theory for the logical entailment relation "|=" of Section 5. The theory consists of twelve inference rules and one axiom. The rules of resolution Tfactoring $\Gamma$ and paramodulation form the core of the deductive system. HoweverTunlike in predicate calculus these three rules are not enough. For a deductive system to be completeTadditional rules for capturing the properties of types and IS-A hierarchies are needed. The large number of inference rules in F-logic compared to predicate calculus stems from the rich semantics of object-oriented systems; this is likely to be the case with any logical system that attempts to adequately capture this paradigm. As will be seen shortlyГmany rules are quite similar to each other Texcept that one may deal with data expressions and the other with signatures or with P-molecules. It is possible to reduce the number of rules by about half through increasing the power of the resolution rule. This is analogous to classical logic where factoring is often combined with resolution. However $\Gamma$ we prefer to keep inference rules simple and increase their number instead $\Gamma$ so that it would be easier to see the rationale behind each rule.

\subsection{Substitutions and Unifiers}

In F-logic $\Gamma m u c h$ of the theory of unifiers carries over from the classical case. HoweverTthis notion needs some adjustments due to the presence of sets and also because of the more flexible syntax of F-molecules (compared to classical logic).

\section{Substitutions}

Let $\mathcal{L}$ be a language with a set of variables $\mathcal{V}$. A substitution is a mapping $\sigma: \mathcal{V} \longmapsto\{$ id-terms of $\mathcal{L}\}$ such that it is an identity everywhere outside some finite set $\operatorname{dom}(\sigma) \subseteq \mathcal{V} \Gamma$ the domain of $\sigma$.

As in classical logicTsubstitutions extend to mappings $\{$ id-terms $\} \longmapsto\{i d$-terms $\}$ as follows:

$$
\sigma\left(f\left(t_{1}, \ldots, t_{n}\right)\right)=f\left(\sigma\left(t_{1}\right), \ldots, \sigma\left(t_{n}\right)\right)
$$

A substitution $\Gamma \sigma \Gamma$ can be further extended to a mapping from molecules to molecules by distributing $\sigma$ through the components of the molecules and applying it to each id-term. For instance $\sigma(Q: P)$ is defined as $\sigma(Q): \sigma(P) \Gamma \sigma(Q:: P)$ as $\sigma(Q):: \sigma(P) \Gamma$ and $\sigma(Q[M t h d @ R, S \rightarrow T])$ is the same as $\sigma(Q)[\sigma(M t h d) @ \sigma(R), \sigma(S) \rightarrow \sigma(T)]$. SimilarlyTsubstitutions extend to F-formulae by distributing them through logical connectives and quantifiers.

A substitution is ground if $\sigma(X) \in U(\mathcal{F})$ for each $X \in \operatorname{dom}(\sigma)$ T that is $\Gamma$ if $\sigma(X)$ has no variables. Given a substitution $\sigma$ and a formula $\varphi \Gamma \sigma(\varphi)$ is called an instance of $\varphi$. It is a ground instance if it contains no variables. A formula is ground if it has no variables. 
(i) For every ground F-molecule $T$, either $T \in \mathbf{T}$ or $\neg T \in \mathbf{T}$.

(ii) A ground clause, $L_{1} \vee \cdots \vee L_{n}$, is in $\mathbf{T}$ if and only if $L_{i} \in \mathbf{T}$, for some $1 \leq i \leq n$.

Proof: Part (i): If $\mathbf{T}$ is finitely satisfiable $\Gamma$ then either $\mathbf{T} \cup\{T\}$ or $\mathbf{T} \cup\{\neg T\}$ is finitely satisfiable. Therefore $\Gamma \mathbf{T}$ must contain either $T$ or $\neg T \Gamma$ since it is maximal. Part (ii) is proved similarly to (i).

Lemma 9.4 Let $\mathbf{T}$ be a maximal finitely satisfiable set of ground clauses. Let $\mathbf{H}$ be the set of all ground molecules in $\mathbf{T}$. Then $\mathbf{H}$ is an H-structure.

Proof: It is easy to see that $\mathbf{H}$ is $=-\operatorname{closed} \Gamma$ since $\mathbf{T}$ is also $=$-closed (or else $\mathbf{T}$ is not maximal).

\section{Theorem 9.5 (cf. Herbrand's Theorem)}

A set of clauses, $\mathbf{S}$, is unsatisfiable if and only if so is some finite subset of ground instances of the clauses in $\mathrm{S}$.

Proof: For the "if" part Tassume that some finite subset of ground clauses of $\mathbf{S}$ is unsatisfiable. Then $\mathbf{S}$ is also unsatisfiable. The "only-if" part is proved by contradiction. Assume that some set of ground instances of the clauses in $\mathbf{S}$ is finitely satisfiable. We will show that then $\mathbf{S}$ is satisfiable.

Let $\mathbf{S}^{\prime}$ be such finitely satisfiable set of ground instances. By Lemma 9.2 Tit can be extended to a maximal finitely satisfiable set $\mathbf{T}$. Let $\mathbf{H}$ be the set of all ground molecules in $\mathbf{T}$ Twhich is an $\mathrm{H}$-structure by Lemma 9.4. We claim that $\mathbf{H} \models C$ if and only if $C \in \mathrm{T} \Gamma$ for every ground clause $C$. Consider the following cases:

(a) $C$ is a ground molecule. By definition $\Gamma \mathbf{H} \models C$ if and only if $C \in \mathbf{T}$;

(b) $C$ is a negative literal $\neg P$. Then $\mathbf{H} \models \neg P$ if and only if $P \notin \mathbf{H}$. Since $\mathbf{H}$ contains all the ground molecules in $\mathbf{T} \Gamma P \notin \mathbf{H}$ if and only if $P \notin \mathbf{T}$. Finally by (i) of Lemma $9.3 \Gamma P \notin \mathbf{T}$ if and only if $\neg P \in \mathrm{T}$;

(c) $C$ is a disjunction of ground literals $L_{1} \vee \cdots \vee L_{n}$. Then

$\mathbf{H}=L_{1} \vee \cdots \vee L_{n}$

if and only if $\mathbf{H}=L_{i}$ for some $i \Gamma$ by definition;

if and only if $L_{i} \in \mathrm{T} \Gamma$ by cases (a) and (b) above;

if and only if $L_{1} \vee \cdots \vee L_{n} \in$ TГby (ii) of Lemma 9.3.

We have $\Gamma$ thus $\Gamma$ shown that $\mathbf{H}$ satisfies every clause of $\mathbf{T}$. Since $\mathbf{S}^{\prime} \subseteq \mathbf{T} \Gamma \mathbf{H}$ is an $\mathbf{H}$-model of $\mathbf{S}$. By Proposition $8.2 \mathrm{TS}$ is satisfiable.

Herbrand's Theorem is a basis for the resolution-based proof theory in classical logic [27]. The next section presents a sound and complete resolution-based proof procedure extending the result of [50]. ThisTin turn $\Gamma$ provides a firm basis for a theory of object-oriented databases and programming. 


\section{Skolemization and Clausal Form}

It is easy to verify that the usual De Morgan's laws hold for F-formulas. Therefore Cevery F-logic formula has a prenex normal form.

Once a formula is converted into an equivalent prenex normal form $\Gamma$ it is Skolemized. Skolemization in F-logic is Tagain $\Gamma$ similar to the classical case $\Gamma$ since id-terms are identical to terms in predicate calculus and because quantification is defined similarly in both cases. For instance $\mathrm{T}$ a Skolem normal form for the molecule $(\forall X \exists Y) g(X, Y)[Y \rightarrow f(X, Y) ; a \rightarrow\{X, Y\}]$ would be $(\forall X) g(X, h(X))[h(X) \rightarrow$ $f(X, h(X)) ; a \rightarrow\{X, h(X)\}]$ Twhere $h$ is a new unary function symbol.

Theorem 9.1 (cf. Skolem's Theorem) Let $\varphi$ be an F-formula and $\varphi^{\prime}$ be its Skolemization. Then $\varphi$ is unsatisfiable if and only if so is $\varphi^{\prime}$.

The proof of this theorem is almost identical to the standard proof for predicate calculus $\mathrm{and}$ is omitted. From now on Twe assume that all formulas are Skolemized. De Morgan's Laws further assure that every formula has a conjunctive and a disjunctive normal form. We can therefore transform every Skolemized formula into a logically equivalent set of clauses $\Gamma$ where clause is a disjunction of literals.

\section{Herbrand's Theorem}

In classical logic $\Gamma$ a set of clauses $\Gamma S \Gamma$ is unsatisfiable if and only if so is some finite set of ground instances of clauses in $\mathbf{S}$; this property is commonly referred to as Herbrand's theorem. In F-logic THerbrand's theorem plays the same fundamental role. We establish this theorem by considering maximal finitely satisfiable setsTsimilarly to the proof of the compactness theorem in [35]. A set $\mathbf{S}$ of ground clauses is finitely satisfiable if every finite subset of $\mathbf{S}$ is satisfiable. A finitely satisfiable set $\mathbf{S}$ is maximal if no other set of ground clauses containing $\mathbf{S}$ is finitely satisfiable. Some useful properties of finitely satisfiable sets are stated below.

Lemma 9.2 Given a finitely satisfiable set of ground clauses, $\mathbf{S}$, there exists a maximal finitely satisfiable set, $\mathbf{T}$, such that $\mathbf{S} \subseteq \mathbf{T}$.

Proof: Let $\Lambda$ be a collection of all finitely satisfiable sets of ground clauses (in a fixed language $\mathcal{L}$ ) that contain $\mathbf{S}$. The set $\Lambda$ is partially ordered by set-inclusion. Since $\mathbf{S} \in \Lambda \Gamma \Lambda$ is non-empty. Furthermore $\Gamma$ for every $\subseteq$-growing chain $\Sigma \subseteq \Lambda$ The least upper bound of the chain $\Gamma \cup \Sigma \Gamma$ is also in $\Lambda$. Indeed:

- $\cup \Sigma$ contains $\mathbf{S}$; and

- $\cup \Sigma$ is finitely satisfiable (for Tif not Tone of the elements of $\Sigma$ must not be finitely satisfiable).

By Zorn's LemmaTthere is a maximal element ГT Tin $\Lambda$.

Lemma 9.3 Let $\mathrm{T}$ be a maximal finitely satisfiable set of ground clauses. 
2. The ordering $\Gamma \prec_{U}$ Tand the class membership relation $\Gamma \epsilon_{U}$ Tare derived from the is-a assertions in $\mathbf{H}$ : For all $[t],[s] \in U$ Twe assert $[s] \preceq_{U}[t]$ if and only if $s:: t \in \mathbf{H} ;$ and $[s] \in_{U}[t]$ if and only if $s: t \in \mathbf{H}$.

3. $I_{\mathcal{F}}(c)=[c]$ Tfor every 0 -ary function symbol $c \in \mathcal{F}$.

4. $I_{\mathcal{F}}(f)\left(\left[t_{1}\right], \ldots,\left[t_{k}\right]\right)=\left[f\left(t_{1}, \ldots, t_{k}\right)\right]$ Tfor every k-ary $(k \geq 1)$ function symbol $f \in \mathcal{F}$.

5. $I_{\rightarrow}^{(k)}([\operatorname{scal} M])\left([o b j],\left[t_{1}\right], \ldots,\left[t_{k}\right]\right)= \begin{cases}{[s]} & \text { if } \operatorname{obj}\left[\operatorname{scal} M @ t_{1}, \ldots, t_{k} \rightarrow s\right] \in \mathbf{H} \\ \text { undefined } & \text { otherwise. }\end{cases}$

6. $I_{\rightarrow}^{(k)}([\operatorname{set} M])\left([o b j],\left[t_{1}\right], \ldots,\left[t_{k}\right]\right)=$

$$
\begin{cases}\left\{[s] \mid \text { where } o b j\left[\operatorname{set} M @ t_{1}, \ldots, t_{k} \rightarrow s\right] \in \mathbf{H}\right\} & \text { if } \operatorname{obj}\left[\operatorname{set} M @ t_{1}, \ldots, t_{k} \rightarrow\{\}\right] \in \mathbf{H} \\ \text { undefined } & \text { otherwise. }\end{cases}
$$

The mappings $I_{\bullet}$ and $I_{\bullet} \rightarrow$ are defined similarly to $(5)$ and $(6) \Gamma$ except that inheritable data expressions must be used instead of the non-inheritable ones.

7. $I_{\Rightarrow}^{(k)}([\operatorname{scal} M])\left([o b j],\left[t_{1}\right], \ldots,\left[t_{k}\right]\right)=$

$$
\begin{cases}\left\{[s] \mid \text { where } \operatorname{obj}\left[\operatorname{scal} M @ t_{1}, \ldots, t_{k} \Rightarrow s\right] \in \mathbf{H}\right\} & \text { if } \operatorname{obj}\left[\operatorname{scal} M @ t_{1}, \ldots, t_{k} \Rightarrow()\right] \in \mathbf{H} \\ \text { undefined } & \text { otherwise. }\end{cases}
$$

8. $I_{\nRightarrow}^{(k)}([\operatorname{set} M])\left([o b j],\left[t_{1}\right], \ldots,\left[t_{k}\right]\right)=$

$$
\begin{cases}\left\{[s] \mid \text { where } o b j\left[\operatorname{set} M @ t_{1}, \ldots, t_{k} \Rightarrow s\right] \in \mathbf{H}\right\} & \text { if } \operatorname{obj}\left[\operatorname{set} M @ t_{1}, \ldots, t_{k} \Rightarrow()\right] \in \mathbf{H} \\ \text { otherwise. }\end{cases}
$$

9. $I_{\wp}(p)=\left\{\left\langle\left[t_{1}\right], \ldots,\left[t_{k}\right]\right\rangle \mid p\left(t_{1}, \ldots, t_{k}\right) \in \mathbf{H}\right\}$.

We remark that in $(6)$ there is a difference between the set $I_{\rightarrow}^{(k)}([\operatorname{set} M])\left([o b j],\left[t_{1}\right], \ldots,\left[t_{k}\right]\right)$ being undefined and being empty. It is undefined if $\mathbf{H}$ contains no atoms of the form $o b j\left[\operatorname{set} M @ t_{1}, \ldots, t_{k} \rightarrow \ldots\right]$ Tnot even $o b j\left[\operatorname{set} M @ t_{1}, \ldots, t_{k} \rightarrow\{\}\right]$. In contrast $\Gamma I_{\rightarrow}^{(k)}([\operatorname{set} M])\left([o b j],\left[t_{1}\right], \ldots,\left[t_{k}\right]\right)$ is empty when $\mathbf{H}$ does contain obj $\left[\operatorname{set} M @ t_{1}, \ldots, t_{k} \rightarrow\{\}\right]$ Cbut has no atoms of the form $\operatorname{obj}\left[\operatorname{set} M @ t_{1}, \ldots, t_{k} \rightarrow\{s\}\right]$ Tfor any $s \in U(\mathcal{F})$. Similar remarks apply to $I_{\nRightarrow}^{(k)}$ in $(7)$ and to $I_{\nRightarrow}^{(k)}$ in $(8)$.

It is easy to see that $\mathbf{I}_{H}=\left\langle U, \prec_{U}, \in_{U}, I_{\mathcal{F}}, I_{\wp}, I_{\rightarrow}, I_{\rightarrow}, I_{\bullet}, I_{\bullet \rightarrow}, I_{\rightrightarrows}, I_{\Rightarrow}\right\rangle$ is well-defined and $\Gamma$ indeed $\Gamma$ is an F-structure. The above correspondence immediately leads to the following result:

Proposition 8.2 Let $\mathbf{S}$ be a set of clauses. Then $\mathbf{S}$ is unsatisfiable if and only if $\mathbf{S}$ has no H-model.

Proof: It is easy to verify that for every $\mathrm{H}$-structure $\mathbf{H} \Gamma$ the entailment $\mathbf{H} \models \mathbf{S}$ takes place if and only if $\mathbf{I}_{H}=\mathbf{S}$ Twhere $\mathbf{I}_{H}$ is the F-structure that corresponds to HTas defined earlier.

\section{Skolemization, Clausal Form, and Herbrand's Theorem}

As in classical logic $\Gamma$ the first step in developing a resolution-based proof theory is to convert all formulas into the prenex normal form and then to Skolemize them. Skolemized formulas are then transformed into an equivalent clausal form. 
Since H-structures are $=$-closed Tit is easy to see that they have closure properties similar to those in Section 7 . These closure properties are obtained from the properties in Section 7 by replacing each $\mathbf{I}=\phi$ there with $\phi \in \mathbf{H}$ Twhere $\phi$ is a molecule $\mathbf{I}$ is an F-structureTand $\mathbf{H}$ is an arbitrary Herbrand structure. For instanceTthe transitivity property for "․ㅡ:

$$
\text { If } \mathrm{I} \models(p \doteq q) \text { and } \mathrm{I} \models(q \doteq r) \text {, then } \mathrm{I} \models(p \doteq r)
$$

now becomes:

$$
\text { If }(p \doteq q) \in \mathbf{H} \text { and }(q \doteq r) \in \mathbf{H} \text {, then }(p \doteq r) \in \mathbf{H}
$$

We can now define truth and logical entailment in H-structures:

Definition 8.1 (Satisfaction of Formulas by $H$-structures) Let $\mathbf{H}$ be an H-structure. Then:

- A ground molecule $t$ Tis true in $\mathbf{H}$ (denoted $\mathbf{H} \models t$ ) if and only if $t \in \mathbf{H}$;

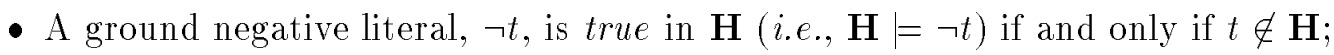

- A ground clause $\Gamma L_{1} \vee \cdots \vee L_{n}$ Tis true in $\mathbf{H}$ if and only if at least one literal $L_{i}$ Tis $\operatorname{true}$ in $\mathbf{H}$;

- A clause $C$ Tis true in $\mathbf{H}$ if and only if all ground instances of $C$ are true in $\mathbf{H}$.

If every clause in $\mathbf{S}$ is true in $\mathbf{H}$ Twe say that $\mathbf{H}$ is a Herbrand model (an H-model) of $\mathbf{S}$.

\section{Correspondence between H-structures and F-structures}

The above observations indicate that $\Gamma$ as in classical logic $\Gamma$ there should be a simple way to construct F-structures out of H-structures $\Gamma$ and vice versa. There is one technical problem $\Gamma$ though: Herbrand universe - the domain of all $\mathrm{H}$-structures - cannot always serve as a domain of an F-structure. Indeed $\mathrm{C}$ in F-structuresTdifferent domain elements represent different objects. However $\mathrm{Tthis}$ is not the case with

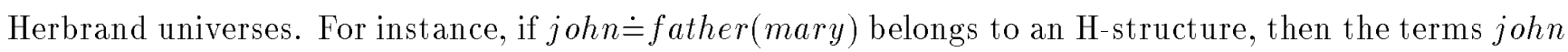
and father(mary) represent the same object Tyet they are different elements of the Herbrand universe.

The same phenomenon is encountered in classical logic with equality 5 and the cure for this problem

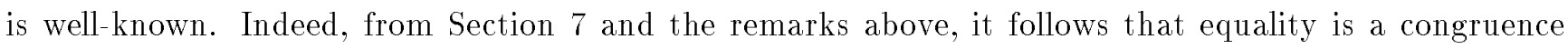
relation on the Herbrand universe. So Twe can construct a domain of an F-structure by factoring $U(\mathcal{F})$ with this relation.

The correspondence between H-structures and F-structures can now be stated as follows: Given an F-structure for a set of clauses ST the corresponding H-structure is the set of ground F-molecules

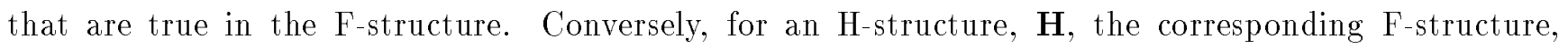
$\mathbf{I}_{H}=\left\langle U, \prec_{U}, \epsilon_{U}, I_{\mathcal{F}}, I_{\wp}, I_{\rightarrow}, I_{\rightarrow}, I_{\bullet}, I_{\bullet}, I_{\rightrightarrows}, I_{\Rightarrow}\right\rangle \Gamma$ is defined as follows: ${ }^{11}$

1. The domain $U$ is $U(\mathcal{F}) / \doteq \Gamma$ the quotient of $U(\mathcal{F})$ induced by the equalities in $\mathbf{H}$. We denote the equivalence class of $t$ by $[t]$.

\footnotetext{
${ }^{11}$ Observe the similarity with the corresponding construction in classical logic with equality.
} 


\section{Trivial object-molecules:}

- For every id-term $\Gamma$ t we have $\mathbf{I}=t[]$

\section{Constituent atoms:}

- For every molecule $\Gamma$ $u \mathbf{I} \mid=u$ if and only if $\mathbf{I} \models v$ holds for every constituent atom $v$ of $u$

The constituent atoms mentioned in the last property are essentially molecules broken into indivisible pieces; they are formally defined as follows:

- Every P-molecule or an is-a assertion is its own constituent atom;

- For an object-molecule $\Gamma G=P[$ method expressions $] \Gamma$ the constituent atoms are:

- For every signature expression $M$ thd $@ Q_{1}, \ldots, Q_{k} \approx\left(R_{1}, \ldots, R_{n}\right)$ in $G$ Twhere $\approx$ is either $\Rightarrow$ or $\Rightarrow$ The corresponding constituent atoms are:

$$
\begin{aligned}
& P\left[M \text { thd } @ Q_{1}, \ldots, Q_{k} \approx()\right] ; \quad \text { and } \\
& P\left[M \text { thd } @ Q_{1}, \ldots, Q_{k} \approx R_{i}\right], \quad i=1, \ldots, n
\end{aligned}
$$

- For every scalar data expression $M t h d @ Q_{1}, \ldots, Q_{k} \sim S$ in $G$ Twhere $\sim$ is either $\rightarrow$ or $\bullet \Gamma$ the corresponding constituent atom is:

$$
P\left[M t h d @ Q_{1}, \ldots, Q_{k} \sim S\right]
$$

- For every set-valued data expression $\Gamma M t h d @ Q_{1}, \ldots, Q_{k} \sim\left\{T_{1}, \ldots, T_{m}\right\}$ in $G \Gamma$ where $\sim$ is either $\rightarrow$ or $\bullet$ Tthe constituent atoms are:

$$
\begin{aligned}
& P\left[\text { Mthd } @ Q_{1}, \ldots, Q_{k} \sim\{\}\right] \text { and } \\
& P\left[\text { Mthd } @ Q_{1}, \ldots, Q_{k} \sim\left\{T_{j}\right\}\right], \quad j=1, \ldots, m
\end{aligned}
$$

The property of scalarity follows from the simple fact that scalar invocations of methods are interpreted via single-valued functions. The other properties are straightforward from Definition 5.1.

\section{Herbrand Structures}

Given an F-logic language $\Gamma \mathcal{L} \Gamma$ with $\mathcal{F}$ as its sets of function symbols $\Gamma$ the Herbrand universe of $\mathcal{L}$ is $U(\mathcal{F}) \Gamma$ the set of all ground id-terms. The Herbrand base of $\mathcal{L} \Gamma \mathcal{H} \mathcal{B}(\mathcal{F})$ Tis the set of all ground molecules (including $\mathrm{P}$-molecules and equality).

Let $\mathbf{H}$ be a subset of $\mathcal{H B}(\mathcal{F})$; it is a Herbrand structure (abbr. $\mathrm{H}$-structure) of $\mathcal{L}$ if and only if it is closed under the logical implication $\Gamma^{\text {" }}=$ " Tintroduced in Section 5 . The requirement of $\mid=$-closure is needed since ground molecules may imply other molecules in a non-trivial way. For instance $\{a:: b, b:: c\} \mid=a:: c$ and $\{a:: b, d[m \Rightarrow a]\} \mid=d[m \Rightarrow b]$. This is reminiscent of the situation in predicate calculus with equality where sets of ground atomic formulas may imply other atomic formulas $(e . g . \Gamma\{a \doteq b, p(a)\} \mid=p(b))$. 
inherited from different superclasses "adds up." For instance $\Gamma$ suppose a class $\Gamma$ cl $\Gamma$ has several signature expressions for the same method $\Gamma m t h d$. These signatures may be specified directly or inherited by $\mathrm{cl}$. Then $\Gamma$ "adding up" means that-as a function invoked on a member of class $c$ - the method mthd belongs to the intersection of all functional types determined by all signatures (whether directly specified or inherited) that $m$ has in $\mathrm{cl}$.

To illustrate $\mathrm{C}$ consider the following example:

$$
\begin{array}{ll}
\text { empl :: person } & \text { person }[\text { name } \Rightarrow \text { string }] \\
\text { assistant :: student } & \text { student }[\text { drinks } \Rightarrow \text { beer; drives } \Rightarrow \text { bargain }] \\
\text { assistant :: empl } & \text { empl }[\text { salary } \Rightarrow \text { int } ; \text { drives } \Rightarrow \text { car }] \\
& \text { assistant }[\text { drives } \Rightarrow \text { oldThing }]
\end{array}
$$

The signature accumulated by assistant from all sources will then be as follows:

$$
\text { assistant }[\text { name } \Rightarrow \text { string; drinks } \Rightarrow \text { beer; drives } \Rightarrow \text { (bargain,car,oldThing); salary } \Rightarrow \text { integer] }
$$

In other words $\Gamma$ an assistant inherits the type of name from the class person $\Gamma$ the type of salary from employee, and his drinking habits come from the class student. The structure of the attribute drives is determined by three factors: (i) the explicitly given signature $\mathrm{i}$ drives $\Rightarrow$ oldThing; and (ii) the signatures inherited from the classes student and empl. The resulting signature states that assistants drive old cars bought at bargain prices.

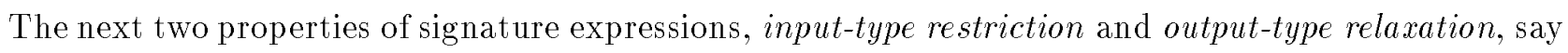
that when methods are viewed as functions They have the usual properties as far as typing is concerned. For instance $\Gamma$ the property input-type restriction says that if a method $\Gamma m t h d \Gamma$ returns values of type $s$ when it is passed arguments of certain typesTthen $m$ thd will still return values of type $s$ when invoked with arguments of more restricted types. Similarly $\mathrm{T}$ the property of output-type relaxation states that if $m$ thd returns a value of some type $r \Gamma$ then this value has also the type $s$ Tor any supertype $s$ of $r$. Output relaxation may seem like an obvious and redundant property. However Tstrictly speaking Tit does not logically follow from other properties.

\subsection{Miscellaneous Properties}

The first property in this category simply states that scalar methods are supposed to return at most one value for any given set of arguments. The second statement says that object molecules that assert no properties are trivially true in all F-structures. The last property is the raison d'être for the name "a molecular formula." According to this propertyГ a molecule that is true in I may spin off a bunch of other TsimplerTformula that are also true in I. MoreoverTthese formulas cannot be decomposed further and $\mathrm{Tin}$ a sense $\mathrm{Tthey}$ can be viewed as subformulas of the original molecule.

\section{Scalarity:}

- If $\mathbf{I}=p\left[S \operatorname{Scal} M @ q_{1}, \ldots, q_{k} \rightarrow r_{1}\right]$ and $\mathbf{I} \mid=p\left[S c a l M @ q_{1}, \ldots, q_{k} \rightarrow r_{2}\right] \Gamma$ then $\mathbf{I}=\left(r_{1} \dot{\doteq} r_{2}\right)$

- If $\mathbf{I} \models p\left[S \operatorname{Scal} M @ q_{1}, \ldots, q_{k} \bullet r_{1}\right]$ and $\mathbf{I} \models p\left[S \operatorname{cal} M @ q_{1}, \ldots, q_{k} \bullet r_{2}\right] \Gamma$ then $\mathbf{I} \models\left(r_{1} \dot{\doteq} r_{2}\right)$ 


\subsection{Properties of the IS-A Relationship}

The following statements say that "::" specifies a partial order on $U(\mathcal{F})$.

IS-A reflexivity:

- $\mathbf{I}=p:: p$

IS-A transitivity:

- If $\mathbf{I} \models p:: q$ and $\mathbf{I} \models q:: r$ then $\mathbf{I} \models p:: r$

IS-A acyclicity:

- If $\mathbf{I} \models p:: q$ and $\mathbf{I} \models q:: p$ then $\mathbf{I} \models(p \doteq q)$

Subclass inclusion:

- If $\mathbf{I} \mid=p: q$ and $\mathbf{I} \mid=q:: r$ then $\mathbf{I} \mid=p: r$

The first three properties are direct consequences of the fact that the relation $\prec_{U}$ on the domain of $\mathbf{I}$ is a partial order; the last property follows from the interplay between $\prec_{U}$ and $\epsilon_{U}$.

\subsection{Properties of Signature Expressions}

In the following rulesTthe symbol $\approx$ denotes either $\Rightarrow$ or $\Rightarrow$. AlsoTin the first two rulesTthe symbol $s$ stands for an element of $U(\mathcal{F})$ or for " ( )" — the empty conjunction of types.

Type inheritance:

- If $\mathbf{I}=p\left[m t h d @ q_{1}, \ldots, q_{k} \approx s\right]$ and $\mathbf{I}=r:: p$ then $\mathbf{I}=r\left[m t h d @ q_{1}, \ldots, q_{k} \approx s\right]$

Input-type restriction:

- If $\mathbf{I}=p\left[m t h d @ q_{1}, \ldots, q_{i}, \ldots, q_{k} \approx s\right]$ and $\mathbf{I}=q_{i}^{\prime}:: q_{i}$ then $\mathbf{I}=p\left[m t h d @ q_{1}, \ldots, q_{i}^{\prime}, \ldots, q_{k} \approx s\right]$ Output-type relaxation:

- If $\mathbf{I}=p\left[m t h d @ q_{1}, \ldots, q_{k} \approx r\right]$ and $\mathbf{I} \mid=r:: s$ then $\mathbf{I}=p\left[m t h d @ q_{1}, \ldots, q_{k} \approx s\right]$

The first two properties directly follow from the anti-monotonicity constraint on the mappings $I_{\Rightarrow}(\nu(m t h d))$ and $I_{\Rightarrow}(\nu(m t h d))$ in Section 5 Twhere $\nu$ is a variable assignment. The last property follows from the upward-closedness of $I_{\Rightarrow}(\nu(m t h d))\left(\nu(p), \nu\left(q_{1}\right), \ldots, \nu\left(q_{k}\right)\right)$ and $I_{\Rightarrow}(\nu(m t h d))\left(\nu(p), \nu\left(q_{1}\right), \ldots, \nu\left(q_{k}\right)\right)$.

\section{Structural Inheritance and Typing}

The above properties of signatures are quite interesting and merit further comments. The first of these properties $\Gamma$ type inheritance (or structural inheritance) $\Gamma$ expresses the fact that structure propagates down from classes to subclasses. This inheritance is monotonic in the sense that any additional structure specified for a subclass is added to the structure inherited from a superclass. MoreoverTany structure 
- $I_{\wp}(\doteq) \stackrel{\text { def }}{=}\{\langle a, a\rangle \mid a \in U\}$.

This obviously implies that if $T$ and $S$ are id-terms $\Gamma$ then $\mathbf{I} \models_{\nu}(T \doteq S)$ if and only if $\nu(T)=\nu(S)$.

To fully integrate P-molecules into F-logic $\Gamma$ we now let $\mathrm{F}$-formulae to be built using any kind of molecules introduced so far. Truth of formulas in F-structures and the notion of a model is defined in an obvious way.

Direct introduction of predicates serves another useful purpose: classical predicate calculus can be now viewed as a subset of F-logic. This has a very practical implication: classical logic programming $\Gamma$ too $\Gamma$ can be thought of as a special case of programming in F-logic. With some additional effort Tmost of classical logic programming theory can be adapted to F-logic 5 which will make it upward-compatible with the existing systems. Section 11 and Appendix A make the first step in this direction.

In this paper $\Gamma$ we do not deal with signatures of $\mathrm{P}$-molecules $\Gamma$ as they are defined and studied in [51Г98].

\section{$7 \quad$ Properties of F-structures}

To get a better grip on the notions developed in the previous sections Twe present a number of simple important properties of F-structures. We express these properties as assertions about logical entailment $\Gamma$

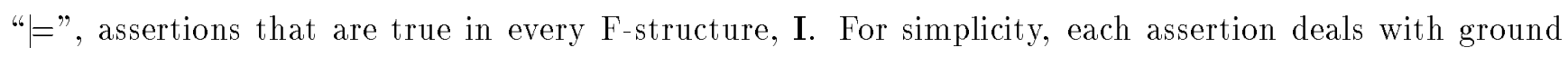
formulas only and is an immediate consequence of the definitions; easy proofs are left as an exercise. As we shall seeГmany of the properties presented herein will form a basis for the inference system of F-logic developed in Section 10.

\subsection{Properties of the Equality}

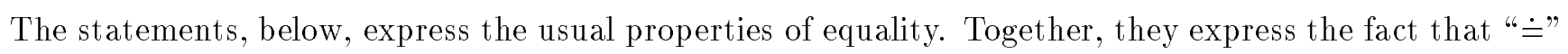
is a congruence relation on $U(\mathcal{F})$.

Reflexivity:

- For all $p \in U(\mathcal{F}) \Gamma \mathbf{I} \models(p \doteq p)$

Symmetry:

- If $\mathbf{I}=(p \dot{\doteq} q)$ then $\mathbf{I} \models(q \dot{=} p)$

Transitivity:

- If $\mathbf{I} \models(p \dot{\doteq} q)$ and $\mathbf{I} \models(q \dot{\doteq} r)$ then $\mathbf{I} \models(p \doteq r)$

\section{Substitution:}

- If $\mathbf{I}=(s \doteq t) \wedge L \Gamma$ and $L^{\prime}$ is obtained by replacing an occurrence of $s$ in $L$ with $t \Gamma$ then $\mathrm{I} \vDash L^{\prime}$ 
with objects leads to a more natural representation. For instanceГa program may mostly manipulate objectsTbut occasionally it may need to verify that certain objects are related via some symmetric relationship (e.g.Tequality or adjacency). Although such relationships can always be encoded as objectsT often this is not the most natural representation. Also representing certain basic relationships $\mathrm{such}$ as equality $\mathrm{T}$ as objects would be extremely awkward. In this section $\Gamma$ we first show how predicates can be encoded as molecules $\Gamma$ and then we incorporate predicate formulas into F-logic $\Gamma$ both syntacticly and semantically.

Predicates can be simulated in several different ways. The approach described here is an adaptation from [50Г 52]. To encode an $n$-ary predicate symbol $\rho$ ए we introduce a new class for which we conveniently use the same symbol $\Gamma p$. Let $p$-tuple be a new n-ary function symbol. We then assert $\left(\forall X_{1} \ldots \forall X_{n}\right)\left(p\right.$-tuple $\left.\left(X_{1}, \ldots, X_{n}\right): p\right)$ and represent classical atoms of the form $p\left(T_{1}, \ldots, T_{n}\right)$ as molecules of the form:

$$
p \text {-tuple }\left(T_{1}, \ldots, T_{n}\right)\left[\arg _{1} \rightarrow T_{1} ; \ldots ; \arg _{n} \rightarrow T_{n}\right]
$$

It is easily seen that the oid template $\Gamma p$-tuple $\left(T_{1}, \ldots, T_{n}\right) \Gamma$ is value-based in the sense of [93] $\Gamma$ that is $\Gamma$ it is fully dependent on the values of their attributes. The term $p$-tuple $\left(T_{1}, \ldots, T_{n}\right)$ can be viewed as an oid of the object to be used to represent a $p$-relationship among $T_{1}, \ldots, T_{n} \Gamma$ if such a relationship exists; Statement (2) above asserts that there is an actual $p$-relationship among $T_{1}, \ldots, T_{n}$.

Although predicates can be represented by objectsTas just described $\Gamma$ to eliminate the need in going through the mental exercise of converting predicates into molecules $\Gamma$ we incorporate them into our syntax and semantics directly.

The expanded language now has a set $\wp$ of predicate symbols. If $p \in \wp$ is an $n$-ary predicate symbol and $T_{1} \Gamma \ldots \Gamma T_{n}$ are id-terms $\Gamma$ then $p\left(T_{1}, \ldots, T_{n}\right)$ is a predicate molecule (abbr.ГP-molecule)

To avoid confusion between id-terms and $\mathrm{P}$-molecules $\Gamma$ we assume that predicates and function symbols belong to two disjoint sets of symbols $\mathrm{T}$ as in classical logic. In a practical logic programming language $\Gamma$ however $\Gamma$ this restriction may not be necessary $\Gamma$ for $\Gamma$ as in Prolog $\Gamma i t$ may be advantageous to overload symbols so that they could be used as predicates and as function symbols at the same time (see Section 16.2).

A (generalized) molecular formula is now either a molecule in the old senseTor a P-molecule (including the case of the equality predicate $e . g . \Gamma T \doteq S$ ). A literal is either a molecular formula or a negated molecular formula.

Predicate symbols are interpreted as relations on $U$ using the function $I_{\wp}$ such that:

- $I_{\wp}(p) \subseteq U^{n} \Gamma$ for any $n$-ary predicate symbol $p \in \wp$.

Given an F-structure $\mathbf{I}=\left\langle U, \prec_{U}, \in_{U}, I_{\mathcal{F}}, I_{\wp}, I_{\rightarrow}, I_{\rightarrow}, I_{\bullet}, I_{\bullet}, I_{\rightrightarrows}, I_{\rightrightarrows}\right\rangle$ and a variable assignment $\nu \Gamma$ we write $\mathbf{I} \models_{\nu} p\left(T_{1}, \ldots, T_{n}\right)$ if and only if

- $\left\langle\nu\left(T_{1}\right), \ldots, \nu\left(T_{n}\right)\right\rangle \in I_{\wp}(p)$.

We also fix a diagonal interpretation for the equality predicate: 
but $c\left[m @ c_{1}, \ldots, c_{k} \Rightarrow()\right]$ is actually false $\Gamma$ then the well-typing conditions of Section 12 mandate that $I_{\rightarrow}(\nu(m))\left(\nu(o), \nu\left(a_{1}\right), \ldots, \nu\left(a_{k}\right)\right)$ must be undefined. This is analogous to a null value of the kind "value inapplicablel" meaning that the value of the invocation of $m$ on $o$ with arguments $a_{1} \Gamma$ $\ldots \Gamma a_{n}$ does not and cannot exist.

- The deceptive simplicity of items (iv) and (v) is a direct result of upward-closedness of the co-domain of $I_{\Rightarrow}(\nu(\operatorname{Scal} M))$ and $I_{\Rightarrow}(\nu(\operatorname{Set} M))$.

Without upward-closedness $\Gamma$ (iv) would have looked thus: $I_{\Rightarrow}^{(n)}(\nu(\operatorname{Scal} M))\left(\nu(O), \nu\left(Q_{1}\right), \ldots, \nu\left(Q_{n}\right)\right)$ must be defined and, for every $i$, there is an element, $u$, in this set such that $u \preceq_{U} \nu\left(R_{i}\right)$. A similar change would have been needed for $(\mathrm{v})$.

A more serious consequence of dumping the upward-closedness condition would be that the elegant anti-monotonicity constraint on $I_{\Rightarrow}(\nu(S$ cal $M))$ and $I_{\Rightarrow}(\nu(S e t M))$ will have to be replaced with a rather awkward condition needed to coerce signatures into behaving as functional types (see Section 7.3).

- Notice how the above definition determines the meaning of molecules of the form $a[M @ X \rightarrow b]$ and $c[M \Rightarrow d]$. The subtlety here is in how method-functions are associated with $M$ - a variable that ranges over methods.

If $\nu$ is a variable assignment $\Gamma$ then $\nu(M)$ is an element in $U$ Tnot in $U(\mathcal{F})$. Therefore associating method-functions with ground id-terms is useless for interpreting the above molecules. This explains why earlier we insisted that $I_{\rightarrow} \Gamma I_{\nRightarrow}$ Tetc. Tmust be functions of the form $U \longmapsto \cdots$ and not of the form $U(\mathcal{F}) \longmapsto \cdots$.

\section{Models and Logical Entailment}

The meaning of the formulae $\varphi \vee \psi \Gamma \varphi \wedge \psi \Gamma$ and $\neg \varphi$ is defined in the standard way: $\mathbf{I} \mid{ }_{\nu} \varphi \vee \psi$ (or

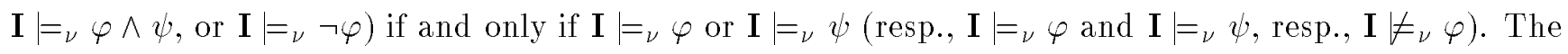
meaning of quantifiers is also standard: $\mathbf{I} \models_{\nu} \psi$ Twhere $\psi=(\forall X) \varphi($ or $\psi=(\exists X) \varphi)$ Tif and only if $\left.\mathbf{I}\right|_{\mu} \psi$ for every (resp. Tsome) $\mu$ that agrees with $\nu$ everywhere Texcept possibly on $X$.

For a closed formula $\Gamma$ Twe can omit the mention of $\nu$ and simply write $\mathbf{I}=\psi$ एsince the meaning of a closed formula is independent of the choice of variable assignments.

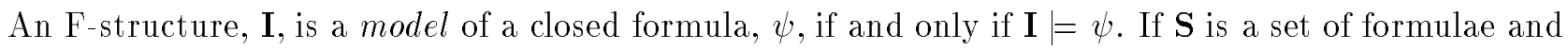
$\varphi$ is a formularwe write $\mathbf{S}=\varphi$ (read: $\varphi$ is logically implied or entailed by $\mathbf{S}$ ) if and only if $\varphi$ is true in every model of $\mathbf{S}$.

\section{Predicates and their Semantics}

It is often convenient to have usual first-order predicates on a par with objects (cf. [31Г50Г3]). This happens when an application is more naturally described in a value-based settingTor when mixing predicates 
(i) $\nu(Q) \preceq_{U} \nu(P)$ Tif $G=Q:: P$; or

$\nu(Q) \in_{U} \nu(P) \Gamma$ if $G=Q: P$.

- When $G$ is an object molecule of the form $O[$ method expressions ] then for every method expression $E$ in $G$ T the following conditions must hold:

(ii) If $E$ is a non-inheritable scalar data expression of the form $S c a l M @ Q_{1}, \ldots, Q_{k} \rightarrow T$ Tthe element $I_{\rightarrow}^{(k)}(\nu(S c a l M))\left(\nu(O), \nu\left(Q_{1}\right), \ldots, \nu\left(Q_{k}\right)\right)$ must be defined and equal $\nu(T)$.

Similar conditions must hold if $E$ is an inheritable scalar data expressionsTexcept that $I_{\rightarrow}^{(k)}$ should be replaced with $I_{\bullet}^{(k)}$.

(iii) If $E$ is a non-inheritable set-valued data expression $\Gamma$ of the form $\operatorname{Set} M @ R_{1}, \ldots, R_{l} \rightarrow$ $\left\{S_{1}, \ldots, S_{m}\right\} \Gamma$ the set $I_{\rightarrow}^{(l)}(\nu(\operatorname{Set} M))\left(\nu(O), \nu\left(R_{1}\right), \ldots, \nu\left(R_{l}\right)\right)$ must be defined and contain the set $\left\{\nu\left(S_{1}\right), \ldots, \nu\left(S_{m}\right)\right\}$.

Similar conditions must hold if $E$ is an inheritable set-valued data expression Texcept that $I_{\rightarrow}^{(k)}$ should be replaced with $I_{\bullet}^{(k)}$.

(iv) If $E$ is a scalar signature expression $\Gamma S c a l M @ Q_{1}, \ldots, Q_{n} \Rightarrow\left(R_{1}, \ldots, R_{u}\right) \Gamma$ then the set $I_{\Rightarrow}^{(n)}(\nu(S \operatorname{Scal} M))\left(\nu(O), \nu\left(Q_{1}\right), \ldots, \nu\left(Q_{n}\right)\right)$ must be defined and contain $\left\{\nu\left(R_{1}\right), \ldots, \nu\left(R_{u}\right)\right\}$.

(v) If $E$ is a set-valued signature expression of the form $\operatorname{Set} M @ V_{1}, \ldots, V_{s} \Rightarrow\left(W_{1}, \ldots, W_{v}\right) \Gamma$ the set $I_{\Rightarrow}^{(s)}(\nu(\operatorname{Set} M))\left(\nu(O), \nu\left(V_{1}\right), \ldots, \nu\left(V_{s}\right)\right)$ must be defined and contain $\left\{\nu\left(W_{1}\right), \ldots, \nu\left(W_{v}\right)\right\}$.

Here (i) says that the object $\nu(Q)$ must be a subclass or a member of the class $\nu(P)$. Conditions (ii) and (iii) say that - in case of a data expression - the interpreting function must be defined on appropriate arguments and yield results compatible with those specified by the expression. Conditions (iv) and (v) say that $\Gamma$ for a signature expression $\Gamma$ the type of a method ( $S$ cal $M$ or $S$ et $M$ ) specified by the expression must comply with the type assigned to this method by I.

The following observations about Definition 5.1 are useful for better understanding the rationale behind certain aspects of the definition of F-structures:

- It follows from (iv) and (v) above that a signature of the form $c\left[m @ c_{1}, \ldots, c_{k} \Rightarrow\right.$ ()] (with nothing inside the parentheses) is not a tautology. Indeed $\Gamma$ there are F-structures in which $I_{\Rightarrow}^{(k)}(\nu(m))\left(\nu(c), \nu\left(c_{1}\right), \ldots, \nu\left(c_{k}\right)\right)$ is undefined. Similarly for $\Rightarrow$.

Such an "empty" signature intuitively says that the respective scalar method is applicable to objects in class $c$ with arguments drawn from classes $c_{1} \Gamma \ldots \Gamma c_{k} \Gamma$ but it does not specify the actual type of the result. Contrapositively $m$ cannot be applied in the above context without the signature $c\left[m @ c_{1}, \ldots, c_{k} \Rightarrow()\right]$ being true. In Section 12 Twe shall see that this enables us to enforce types by withholding signatures for certain method invocations.

- The case when $c\left[m @ c_{1}, \ldots, c_{k} \Rightarrow()\right]$ is true in an F-structureГITbut $I_{-}(\nu(m))\left(\nu(o), \nu\left(a_{1}\right), \ldots, \nu\left(a_{k}\right)\right)$ is undefined Twhere $\nu(o) \epsilon_{U} \nu(c)$ and $\nu\left(a_{1}\right) \in_{U} \nu\left(a_{1}\right) \Gamma \ldots \Gamma \nu\left(a_{k}\right) \in_{U} \nu\left(a_{k}\right)$ Tis analogous to a null value of the kind "missing value" known from the database theory [94]. When the above value is defined 
$v=I_{\rightarrow}^{(k)}(m)(o, \overrightarrow{a r g} s)$ is defined $\Gamma$ it must belong to every class in $I_{\Rightarrow}^{(k)}(m)(c l, \overrightarrow{t y p e s}) \Gamma i . e . \Gamma v \in_{U} w$ for each $w \in I_{\Rightarrow}^{(k)}(m)(c l, t y p e s)$. In particularTa set of types is interpreted essentially as an extended "and" of its members. This is the reason for the notation "(...)" in the signatures (cf. Figure 4).

Similarly the meaning of $I_{\Rightarrow}^{(k)}(m)$ is defined to be the type of the set-valued function $I_{\rightarrow}^{(k)}(m)$. In this

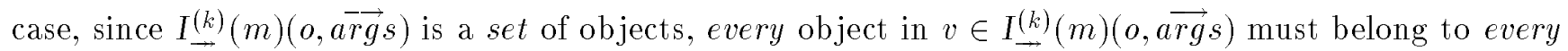
class $\Gamma$ Tin $I_{\nRightarrow}^{(k)}(m)($ cl,types $)$ Гi.e. Tthe relationship $v \in_{U} w$ must hold.

The rationale behind the anti-monotonicity and the upward-closedness conditions on $I_{\Rightarrow}^{(k)}(m)$ and $I_{\Rightarrow}^{(k)}(m)$ now follows from the intended meaning of these functions. For instance $\mathrm{C}$ if the object $I_{\rightarrow}^{(k)}(m)(o, \overrightarrow{a r g} s)$ is of type $c l\left(\right.$ i.e. $\left.\Gamma I_{\rightarrow}^{(k)}(m)(o, a \overrightarrow{r g} s) \in_{U} c l\right)$ then $\Gamma$ clearly $\Gamma I_{\rightarrow}^{(k)}(m)(o, a \overrightarrow{r g} s)$ must be a member of every superclass of $c l\left(i . e . \Gamma I_{\rightarrow}^{(k)}(m)(o, a \overrightarrow{r g} s) \in_{U} c l^{\prime}\right.$ Tfor every $c l^{\prime}$ such that $\left.c l \prec_{U} c l^{\prime}\right)$; thus $I_{\Rightarrow}^{(k)}(m)(c l, t y p e s)$ must be upward-closed. Similarly if $I_{\rightarrow}^{(k)}(m)$ can be invoked on every member of class $c l$ with proper arguments of type types $\overrightarrow{t h e n} m$ must also be invocable on every member $\Gamma$ $o$ of any subclass $c l^{\prime}$ of

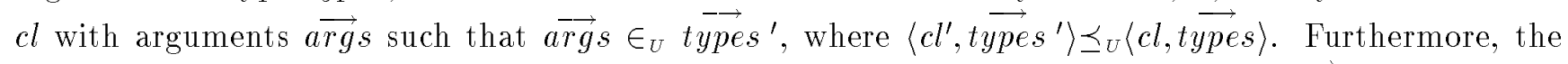
result of this application $\Gamma v=I_{\rightarrow}^{(k)}(m)(\langle o, \overrightarrow{a r g} s\rangle) \Gamma$ must still be typed by $I_{\Rightarrow}^{(k)}(m)(c l, t y p e s)$. Now $\overrightarrow{\text { since }}$

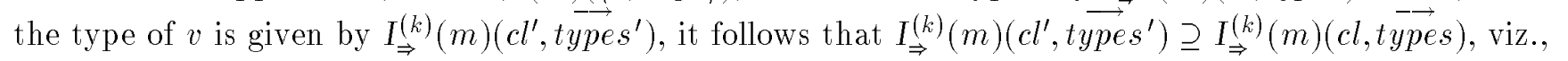
anti-monotonicity.

The above relationship between $I_{\Rightarrow} \Gamma I_{\Rightarrow}$ and $I_{\rightarrow} \Gamma I_{\rightarrow}$ is not part of the definition of F-structures. Instead $\Gamma$ it is captured at the meta-level $\mathrm{C}$ by the definition of type-correctness in Section 12. The relationship between $I_{\Rightarrow} \Gamma I_{\Rightarrow}$ and $I_{\bullet}, \Gamma I_{\bullet}$, Tis also captured by the notion of type-correctness. HoweverTit is slightly different from the relationship between $I_{\Rightarrow} \Gamma I_{\Rightarrow}$ and $I_{\rightarrow} \Gamma I_{\rightarrow}$ described above; it is fully explained in Section 12.

\subsection{Satisfaction of F-formulas by F-structures}

A variable assignment $\nu$ Tis a mapping from the set of variables $\Gamma \mathcal{V}$ Tto the domain $U$. Variable assignments extend to id-terms in the usual way: $\nu(d)=I_{\mathcal{F}}(d)$ if $d \in \mathcal{F}$ has arity 0 and $\Gamma$ recursively $\nu(f(\ldots, T, \ldots))=$ $I_{\mathcal{F}}(f)(\ldots, \nu(T), \ldots)$.

\section{Molecular Satisfaction}

Let $\mathbf{I}$ be an F-structure and $\nu$ a variable assignment. Intuitively $\mathrm{T}$ a molecule $T[\cdots]$ is true under $\mathbf{I}$ with respect to a variable assignment $\nu$ Tdenoted $\mathbf{I} \models_{\nu} T[\cdots]$ Tif and only if the object $\nu(T)$ in $\mathbf{I}$ has properties that the formula $T[\cdots]$ says it has. As a special case $\Gamma$ molecules of the form $T[] \Gamma$ which specify no properties $\Gamma$ come out as tautologies. An is-a molecule $P:: Q$ or $P: Q \Gamma$ is true if the objects involved $\Gamma$ $\nu(P)$ and $\nu(Q)$ Tare related via $\prec_{U}$ or $\epsilon_{U}$ to each other.

Definition 5.1 (Satisfaction of F-Molecules) Let I be an F-structure and $G$ be an F-molecule. We write $\mathbf{I} \models{ }_{\nu} G$ if and only if all of the following holds:

- When $G$ is an is-a assertion then: 


\section{Attachment of Types to Methods - The Mappings $I_{\Rightarrow}$ and $I_{\Rightarrow}$}

Since methods are interpreted as functions Tthe meaning of a signature expressions must be a functional type for its role is to specify the type of a method. To specify a functional typeГone must describe the types of the arguments to which the function can be applied and the types of the results returned by the function. FurthermoreTthe description should account for polymorphic types (cf. (1) earlier).

Model-theoretically $\Gamma$ functional types are described via mappings $\Gamma I_{\Rightarrow}$ and $I_{\Rightarrow} \Gamma$ that cast tuples of objects to sets of objects $\Gamma$ where objects are considered in their role as classes. These mappings must satisfy the usual properties of polymorphic functional types $\Gamma$ as spelled out below. As in the case of $I_{\rightarrow}$ and related functions $\Gamma$ these mappings are attached to the elements of $U$ rather than $U(\mathcal{F})$.

We start with a very succinct definition of $I_{\Rightarrow}$ and $I_{\Rightarrow}$ and then follow with a discussion of the properties of these mappings. ${ }^{9}$

- $I_{\Rightarrow}: U \longmapsto \prod_{i=0}^{\infty}$ PartialAntiMonotone $\prec_{U}\left(U^{i+1}, \mathcal{P}_{\uparrow}(U)\right)$

- $I_{\Rightarrow}: U \longmapsto \prod_{i=0}^{\infty}$ Partial AntiMonotone $\prec_{U}\left(U^{i+1}, \mathcal{P}_{\uparrow}(U)\right)$

Here $\mathcal{P}_{\uparrow}(U)$ is a set of all upward-closed subsets of $U$. A set $V \subseteq U$ is upward closed if $v \in V$ and $v \prec_{U} v^{\prime}$ (where $v^{\prime} \in U$ ) imply $v^{\prime} \in V$. When $V$ is viewed as a set of classes $\Gamma$ upward closedness simply means that $\Gamma$ along with each class $v \in V \Gamma$ this set also contains all the superclasses of $v$. Partial AntiMonotone $\prec_{U}\left(U^{i+1}, \mathcal{P}_{\uparrow}(U)\right)$ denotes the set of partial anti-monotonic functions from $U^{i+1}$ to $\mathcal{P}_{\uparrow}(U)$. For a partial function $\rho: U^{k} \longmapsto \mathcal{P}_{\uparrow}(U) \Gamma$ anti-monotonicity means that if $\vec{u}, \vec{v} \in U^{k} \Gamma \vec{v} \preceq_{U} \vec{u} \Gamma$ and $\rho(\vec{u})$ is defined Then $\rho(\vec{v})$ is also defined and $\rho(\vec{v}) \supseteq \rho(\vec{u}) .{ }^{10}$

\section{Discussion: The Relationship between $I_{\rightarrow}$ and $I_{\Rightarrow}$}

The definition of F-structures is now complete. In the rest of this subsectionTwe discuss the properties of $I_{\Rightarrow}$ and $I_{\Rightarrow}$ and show that they coincide with the standard properties of functional types (see $\Gamma$ e.g. $[[25]) \Gamma$ albeit expressed in a differentTmodel-theoretic notation. As with $I_{\rightarrow} \Gamma$ we use $I_{\Rightarrow}^{(k)}(m)$ to refer to the $k$-th component of the tuple $I_{\Rightarrow}(m)$. We use similar notation $\Gamma I_{\nRightarrow}^{(k)}(m) \Gamma$ for set-valued methods.

The intended meaning of $I_{\rightarrow}^{(k)}(m)$ is the type of the $(k+1)$-ary function $I_{\rightarrow}^{(k)}(m)$. In other words $\Gamma$ the domain of definition of $I_{\Rightarrow}^{(k)}(m)$ should be viewed as a set of $(k+1)$-tuples of classes $\left\langle\right.$ host-cl, arg-type ${ }_{1}, \ldots$, arg-type $\left._{k}\right\rangle \Gamma$ that type tuples of $\operatorname{arguments} \Gamma\left\langle o, \arg _{1}, \ldots, \arg _{k}\right\rangle \Gamma$ on which $I_{\rightarrow}^{(k)}(m)$ can be invoked (i.e. $\Gamma$ argument-tuples such that $\left\langle o, \arg _{1}, \ldots, \arg _{k}\right\rangle \epsilon_{U}\left\langle\right.$ host-cl, arg-type ${ }_{1}, \ldots$, arg-type $\left._{k}\right\rangle$ holds). For every tuple of classes $\Gamma\langle c l, t \overrightarrow{y p e} s\rangle \in U^{k+1} \Gamma$ if $I_{\Rightarrow}^{(k)}(m)(c l, t \overrightarrow{y p e s})$ is defined $\Gamma$ it represents the type of $I_{\rightarrow}^{(k)}(m)(o, a \vec{r} g s)$ for any tuple of arguments such that $\langle o, \overrightarrow{a r g} s\rangle \in_{U}\langle$ cl,types $\rangle$. This means that if

\footnotetext{
${ }^{9}$ Signatures constitute a fairly advanced level of F-logic; its basic features-is-a hierarchy and data expressions-do not depend on the specifics of the above type system. For this reason, on the first reading it may be possible to skip signature-related aspects of F-logic, including the remaining part of this subsection.

${ }^{10}$ Actually, these functions are monotone with respect to Smyth's ordering [24]. For upward-closed sets, $S \subseteq^{s m y t h} S^{\prime}$ if and only if $S \supseteq S^{\prime}$.
} 
whether the method is invoked as a scalar or a set-valued function. Therefore to assign meaning to methodsTan F-structure has to attach an appropriate function to each method.

As explained in Section 3Г F-logic allows any id-term to be used as a method name. HenceГ we need to associate a function to each id-term. HoweverTto provide for higher-order jobs $\mathrm{such}$ as schema manipulation $\Gamma$ one has to allow variables to range over methods. It turns out that to give meaning to molecules with method-variables $\Gamma$ it is necessary to associate these functions with each element of $U$ Tnot $U(\mathcal{F}) .^{8}$ Furthermore ${ }^{2}$ since methods can have different arities $\Gamma$ we need to associate a function for each possible arity.

Formally in their role as methodsTobjects are interpreted via an assignment of appropriate functions to each element of $U \Gamma$ using the maps $I_{\rightarrow} \Gamma I_{\bullet} \Gamma I_{\rightarrow} \Gamma$ and $I_{\bullet}$. More precisely $\Gamma$ for every object $\Gamma$ its incarnation as a scalar method is obtained via the mapping

$$
\text { - } I_{\rightarrow}, I_{\bullet}: U \longmapsto \prod_{k=0}^{\infty} \operatorname{Partial}\left(U^{k+1}, U\right)
$$

Each of these mappings associates a tuple of partial functions $U^{k+1} \rightarrow U$ with every element of $U$; there is exactly one such function in the tupleIfor every method-arity $k \geq 0$. In other wordsTthe same method can be invoked with different arities.

In addition to different aritiesTevery method can be invoked as a scalar or as a set-valued function (cf. empl[jointWorks@bill $\rightarrow X]$ and student [jointWorks @bill $\rightarrow X]$ in Section 3). Semantically this is achieved by interpreting the set-valued incarnations of methods separately

- $I_{\rightarrow}, I_{\bullet}: U \longmapsto \prod_{k=0}^{\infty} \operatorname{Partial}\left(U^{k+1}, \mathcal{P}(U)\right)$

For every method-arity $k$ Teach of these mappings associates a partial function $U^{k+1} \rightarrow \mathcal{P}(U)$ with each element of $U$. Note that each element of $U$ has four different sets of interpretations: two provided by $I_{\rightarrow}$ and $I_{\bullet}$ and two provided by $I_{\rightarrow}$ and $I_{\bullet}$.

The difference between the " $\rightarrow$ "-versions and the " $\rightarrow$ "-versions in the above mappings is that " $\rightarrow$ "versions are used to interpret inheritable data properties $\Gamma$ while “ $\bullet$ "-versions are for non-inheritable data properties.

As seen from the above definitions $\Gamma I_{\rightarrow}(m)$ (and $I_{\rightarrow}(m) \Gamma I_{\bullet}(m) \Gamma I_{\bullet}(m)$ ) where $m \in U$ Tis an infinite tuple of functions parameterized by the arity $k \geq 0$. To refer to the $k$-th component of such a tuple we use the notation $I_{\rightarrow}^{(k)}(m)\left(\operatorname{resp} . \Gamma I_{\rightarrow}^{(k)}(m) \Gamma I_{\bullet}(m)\right.$ or $\left.I_{\bullet}(m)\right)$. Thus $\Gamma$ a method $\Gamma m \Gamma$ that occurs in a scalar non-inheritable data expression with $k$ proper arguments is interpreted by $I_{\rightarrow}^{(k)}(m)$; if $m$ occurs in a

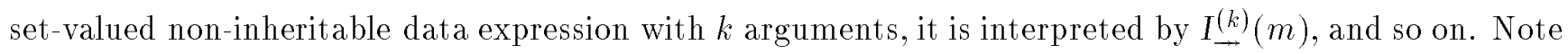
that $I_{\rightarrow}^{(k)}(m)$ and the other three mappings are $(k+1)$-ary functions. The first argument here is the host object of the invocation of the method $m$; the other $k$ arguments correspond to the proper arguments of the invocation. In the parlance of object-oriented systemsTan expression $\Gamma$ such as $I_{\rightarrow}^{(k)}(m)\left(o b j, a_{1}, \ldots, a_{k}\right) \Gamma$ is interpreted as a request to object $o b j$ to invoke a scalar method $\Gamma m \Gamma$ on the arguments $a_{1}, \ldots, a_{k}$.

\footnotetext{
${ }^{8}$ This is a subtle technical point whose necessity will become apparent after the notion of satisfaction in F-structures is introduced.
} 


\section{Semantics}

Given a pair of sets $U$ and $V$ Twe shall use Total $(U, V)$ to denote the set of all total functions $U \vdash V$; similarly $\operatorname{Partial}(U, V)$ stands for the set of all partial functions $U \longmapsto V$. The power-set of $U$ will be denoted by $\mathcal{P}(U)$. FurtherTgiven a collection of sets $\left\{S_{i}\right\}_{i \in \mathcal{N}}$ parameterized by natural numbers $\Gamma \prod_{i=1}^{\infty} S_{i}$ will denote the Cartesian product of the $S_{i}$ 's $\Gamma$ that is $\Gamma$ the set of all infinite tuples $\left\langle s_{1}, \ldots, s_{n}, \ldots\right\rangle$.

\section{$5.1 \quad$ F-structures}

In F-logic $\Gamma$ semantic structures are called F-structures. Given a language of F-logic $\Gamma \mathcal{L}$ - an F-structure is a tuple $\mathbf{I}=\left\langle U, \prec_{U}, \epsilon_{U}, I_{\mathcal{F}}, I_{\rightarrow}, I_{\rightarrow}, I_{\bullet}, I_{\bullet}, I_{\rightrightarrows}, I_{\rightrightarrows}\right\rangle$. Here $U$ is the domain of $\mathrm{I} \Gamma \prec_{U}$ is a partial order on $U$ Tand $\epsilon_{U}$ is a binary relation. As usual $\Gamma$ we write $a \preceq_{U} b$ whenever $a \prec_{U} b$ or $a=b$. We extend $\preceq_{U}$ and $\epsilon_{U}$ to tuples over $U$ in a natural way: for $\vec{u}, \vec{v} \in U^{n}$ and $S \subseteq U^{n}$ Twe write $\vec{u} \preceq_{U} \vec{v}$ or $\vec{u} \in_{U} \vec{v}$ if the corresponding relationships hold between $\vec{u}$ and $\vec{v}$ component-wise.

The ordering $\prec_{U}$ on $U$ is a semantic counterpart of the subclass relationship $\Gamma$ i.e. $\Gamma a \prec_{U} b$ is interpreted as a statement that $a$ is a subclass of $b$. The binary relation $\epsilon_{U}$ will be used to model class membership $\Gamma$ i.e. $\Gamma a \epsilon_{U} b$ should be taken to mean that $a$ is a member of class $b$. The two binary relationships $\Gamma \prec_{U}$ and $\epsilon_{U}$ Tare related as follows: If $a \epsilon_{U} b$ and $b \preceq_{U} c$ then $a \epsilon_{U} c$. This is just another way of saying that the extension of a subclass (i.e. Tits set of members) is a subset of the extension of a superclass.

We do not impose any other restrictions on the class membership relation to accommodate the widest range of applications. In particular $\Gamma \epsilon_{U}$ does not have to be acyclic and even $s \epsilon_{U} s$ is a possibility (i.e. $\Gamma$ a class may be a member of itself when viewed as an object). ${ }^{7}$ The reader should not be misled into thinking that $v$ in $u \epsilon_{U} v$ is a subset of $U$ that contains $u$. The actual meaning of such a statement is that $v$ is an element of $U$ that denotes a subset of $U$ Tand $u$ is a member of this subset.

By analogy with classical logic $\Gamma$ we can view $U$ as a set of all actual objects in a possible world $\Gamma \mathrm{I}$. Ground id-terms (the elements of $U(\mathcal{F})$ ) play the role of logical object id's. They are interpreted by the objects in $U$ via the mapping $I_{\mathcal{F}}: \mathcal{F} \longmapsto \cup_{i=0}^{\infty} \operatorname{Total}\left(U^{i}, U\right)$. This mapping interprets each k-ary object constructor $\Gamma f \in \mathcal{F} \Gamma$ by a function $U^{k} \longmapsto U$. For $k=0 \Gamma I_{\mathcal{F}}(f)$ can be identified with an element of $U$. ThusTfunction symbols are interpreted the same way as in predicate calculus.

The remaining six symbols in I denote mappings for interpreting each of the six types of method expressions in F-logic. These mappings are described next.

\section{Attachment of Functions to Methods — The Mappings $I_{\rightarrow}, I_{\rightarrow}, I_{\bullet}$, and $I_{\bullet} \rightarrow$}

As in classical logic $\Gamma$ the mapping $I_{\mathcal{F}}$ above is used to associate an element of $U$ with each id-term. HoweverTid-terms can also be used to denote methods. A method is a function that takes a host object and a list of proper arguments and maps them into another object or a set of objects $\Gamma$ depending on

\footnotetext{
${ }^{7}$ Such flexibility is sometimes required in AI applications, where $s$ would be interpreted as a "typical" element of class $s$.
} 
It follows from the syntax that every logical id can denote an entity or a method $\Gamma$ depending on the syntactic position of this id within the formula. In an occurrence as a method $\mathrm{Tthis}$ id can denote either a scalar function or a set-valued function. The type of the invocation (scalar or set-valued) is determined by the contextrviz., by the type of the associated arrow.

Is-a assertions are always atomic - they cannot be decomposed further into simpler formulas. Other molecular formulas ThoweverTare not always atomic. As will be seen shortly Ta molecule such as $X\left[a_{t t r} \rightarrow\right.$ $\left.a ; a t t r_{2} \rightarrow Y\right]$ is equivalent to a conjunction of its atoms $\Gamma X\left[a t t r_{1} \rightarrow a\right] \wedge X\left[a t t r_{2} \rightarrow Y\right]$. It is for this property that we call such formulas "molecular" instead of "atomic." Atomic formulas will be defined laterTin Section 7.

\section{Complex Formulas}

F-formulae are built up from simpler F-formulae by means of logical connectives and quantifiers:

- Molecular formulae are F-formulas;

- $\varphi \vee \psi \Gamma \varphi \wedge \psi \Gamma \neg \varphi$ are F-formulaeГif so are $\varphi$ and $\psi$;

- $\forall X \varphi \Gamma \exists Y \psi$ are formulae $\Gamma$ if so are $\varphi \Gamma \psi$ and $X \Gamma Y$ are variables.

In addition Twe define a literal to be either a molecular formula or a negation of a molecular formula.

In Section 3 and elsewhere in this paper $\Gamma$ we shall often use the implication connective $" \leftarrow$ ". In F-logic $\Gamma$ this connective is defined as in classical logic: $\varphi \leftarrow \psi$ is just another way of saying $\varphi \vee \neg \psi$. There is a tradition to refer to logical statements written in the implicative form as rules. This terminology was already used in the example of Section 3Tand we shall continue this practice.

It is sometimes convenient to combine different kinds of molecules (as in (vii) $\Gamma$ (viii) and (ix) of Figure 4) and write $\mathrm{say} \Gamma$

$$
Q: P[S \operatorname{cal} M @ X \rightarrow(Y: S) ; \operatorname{Set} M @ Y, W \Rightarrow(Z: R, T)]
$$

as a short-hand for

$$
Q: P \wedge Q[\text { ScalM } @ X \rightarrow Y] \wedge Y: S \wedge Q[\text { Set } M @ Y, W \Rightarrow(Z, T)] \wedge Z: R .
$$

FurthermoreFeven though the symbols on the right-hand side of the arrows denote id-terms by definition $\Gamma$ it is often convenient (and Tin factTcustomary) to combine molecules as in (i) and (ii) of Figure 4 . For instance $\Gamma$

$$
P[S \operatorname{cal} M @ X \rightarrow Q[S e t M @ Y \rightarrow\{T, S\}]]
$$

can be defined as a short-hand for

$$
P[S \operatorname{cal} M @ X \rightarrow Q] \wedge Q[\text { Set } M @ Y \rightarrow\{T, S\}]
$$


In data expressions $\Gamma$ the terms $T$ and $S_{i}$ are id-terms that represent output of the respective methods $\Gamma$ ScalarMethod and SetMethod when they are invoked on the host-object $O$ with the arguments $Q_{1} \Gamma \ldots \Gamma$ $Q_{k}$ and $R_{1} \Gamma \ldots \Gamma R_{l}$ Trespectively. The arguments are id-terms.

In signature expressions $\Gamma A_{i}$ and $B_{j}$ are id-terms that represent types of the results returned by the respective methods when invoked on an object of class $C$ with arguments of types $V_{1} \Gamma \ldots \Gamma V_{n}$ and $W_{1} \Gamma$ $\ldots \Gamma W_{s}$ Trespectively; these arguments are also id-terms. The notation $(\cdots)$ in signature expressions is intended to say that the output of the method must belong to all the classes listed to the right of " $\Rightarrow$ " or " $\Rightarrow "$.

The order of data and signature expressions in a molecule is immaterial. For convenience the same method and even the same data/signature expression may have multiple occurrences in the same molecule. Likewise Tthe same id-term may occur multiple times inside the braces in a dataTor signature expression.

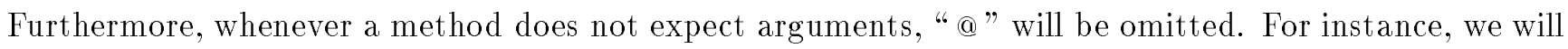
write $P[M t h d \rightarrow V a l]$ instead of $P[M t h d @ \rightarrow V a l]$ Tand similarly for $\rightarrow \Gamma \Rightarrow \Gamma$ and $\Rightarrow$. Likewise $\rightarrow$ when

only one element appears inside \{\} Twe may write $P[\ldots \rightarrow S]$ instead of $P[\ldots \rightarrow\{S\}]$; we shall also write $Q[\ldots \Rightarrow T]$ and $Q[\ldots \Rightarrow T]$ instead of $Q[\ldots \Rightarrow(T)]$ Tand $Q[\ldots \Rightarrow(T)]$.

\section{Discussion}

Informally Tan object molecule in (ii) above asserts that the object denoted by $O$ has properties specified by the method expressions. Data expressions are used to define properties of objects in terms of what their methods are supposed to do. Inheritable data expressions may be inherited by subclasses and individual members of the object (when it plays the role of a class); by contrast properties specified as non-inheritable cannot be inherited. A signature expression in (ii) specifies type constraints on the methods applicable to the objects in class $O$. Typing is given both for method arguments and for their results. For instanceГthe scalar signature expression in (ii) says that Scalar Method is a scalar method and that when it is invoked on a host-object of class $O$ with proper arguments coming from classes $V_{1} \Gamma \ldots \Gamma$ $V_{n}$ Tthen the result must simultaneously belong to classes $A_{1} \Gamma \ldots \Gamma A_{r}$. Similarly The typing for Set Method in (ii) says that it is a set-valued method; when it is invoked on a host object of class $O$ with proper arguments of classes $W_{1} \Gamma \ldots \Gamma W_{s}$ Tthen each element in the resulting set must simultaneously belong to the classes $B_{1} \Gamma \ldots \Gamma B_{t}$.

Notice that a molecule $\Gamma$ such as $a[a t t r \rightarrow b ;$ attr $\rightarrow\{c, d\} ;$ attr $\bullet e] \Gamma$ is syntactically well-formed despite the fact that attr is used to specify a non-inheritable scalar property of the object $a$ due to one part of the moleculeTa non-inheritable set-valued property due to anotherTand also an inheritable scalar property due to the third part. This apparent contradiction is easily resolved at the semantic level: The attribute attr has value $b$ on object $a$ when invoked as a non-inheritable scalar method (with the arrow “ $\rightarrow$ "); it returns the set $\{c, d\}$ when called as a non-inheritable set-valued method (with " $\rightarrow$ "); and it returns the value $e$ when called as an inheritable scalar method (with the arrow " $\bullet$ "). If $a$ happens to be a class-object $\Gamma$ the properties attr $\rightarrow b$ and attr $\rightarrow\{c, d\}$ are not inheritable by the members and subclasses of $a$. However $\Gamma$ attr $\bullet e$ is inheritable $\Gamma$ as it is specified as such. 
In this paperTwe adopt a convention inspired by Prolog syntaxTwhereby a symbol that begins with a lower-case letter denotes a ground id-term and a symbol that begins with a capital letter denotes an id-term that may be non-ground.

Definition 4.1 (Molecular Formulas) A molecule in F-logic is one of the following statements:

(i) An is-a assertion of the form $C:: D$ or of the form $O: C$ Twhere $C \Gamma D$ Tand $O$ are id-terms.

(ii) An object molecule of the form $O$ [ semicolon-separated list of method expressions ].

A method expression can be either a non-inheritable data expression $\Gamma$ an inheritable data expression Tor a signature expression.

- Non-inheritable data expressions take one of the following two forms:

- A non-inheritable scalar expression $(k \geq 0)$ :

$$
\text { ScalarMethod } @ Q_{1}, \ldots, Q_{k} \rightarrow T
$$

- A non-inheritable set-valued expression $(l, m \geq 0)$ :

$$
\text { SetMethod } @ R_{1}, \ldots, R_{l} \rightarrow\left\{S_{1}, \ldots, S_{m}\right\}
$$

- Inheritable scalar and set-valued data expressions are like non-inheritable expressions except that " $\rightarrow$ " is replaced with " $\rightarrow$ " and " $\rightarrow$ " is replaced with $\bullet$.

- Signature expressions also take two forms:

- A scalar signature expression $(n, r \geq 0)$ :

$$
\text { Scalar Method } @ V_{1}, \ldots, V_{n} \Rightarrow\left(A_{1}, \ldots, A_{r}\right)
$$

- A set-valued signature expression $(s, t \geq 0)$ :

$$
\text { SetMethod } @ W_{1}, \ldots, W_{s} \Rightarrow\left(B_{1}, \ldots, B_{t}\right)
$$

The first is-a assertion in (i) $\Gamma C:: D$ Tstates that $C$ is a nonstrict subclass of $D$ (i.e. Tinclusive of the case when $C$ and $D$ denote the same class). ${ }^{6}$ The second assertion $\Gamma O: C$ Tstates that $O$ is a member of class C.

In (ii) $\Gamma O$ is an id-term that denotes an object. ScalarMethod and SetMethod are also id-terms. However The syntactic position of Scalar Method indicates that it is invoked on $O$ as a scalar methodI while the syntactic position of SetMethod indicates a set-valued invocation. (If Scalar Method and Set Method have variablesTeach of these terms denotes a family of methods rather than a single method.) Double-headed arrows $\Gamma \rightarrow \Gamma \bullet \Gamma$ and $\Rightarrow$ indicate that SetMethod denotes a set-valued function. The single-headed arrows $\Gamma \rightarrow \Gamma \bullet$ Tand $\Rightarrow$ Tindicate that the corresponding method is scalar.

\footnotetext{
${ }^{6}$ Assertions, such as person :: person, will later turn out to be tautologies, i.e., any class is a subclass of itself.
} 
Despite the higher-order syntax The underlying semantics of F-logic formally remains first-order ${ }^{5}$ which explains why it is possible to circumvent difficulties normally associated with higher-order theories. These important issues are beyond the scope of this paper; a more complete discussion appears in [30].

\section{Syntax}

The alphabet of an F-logic language $\Gamma \mathcal{L}$ Consists of:

- a set of object constructors $\mathcal{F}$;

- an infinite set of variables $\mathcal{V}$;

- auxiliary symbols $\Gamma$ such as $\Gamma(\Gamma) \Gamma[\Gamma] \Gamma \rightarrow \Gamma \rightarrow \Gamma \bullet \Gamma \bullet \Gamma \Rightarrow \Gamma \Rightarrow \Gamma$ etc; and

- usual logical connectives and quantifiers $\Gamma \vee \Gamma \wedge \Gamma \neg \Gamma \leftarrow \Gamma \forall \Gamma \exists$.

Object constructors (elements of $\mathcal{F}$ ) play the role of function symbols of F-logic. Each function symbol has an arity - a nonnegative integer that determines how many arguments this symbol can take. Symbols of arity 0 play the role of constant symbols; symbols of arity $\geq 1$ are used to construct larger terms out of simpler ones. An id-term is a usual first-order term composed of function symbols and variablesTas in predicate calculus. The set of all ground (i.e. Tvariable-free) id-terms is denoted by $U(\mathcal{F})$. This set is also commonly known as Herbrand Universe.

Conceptually ground id-terms play the role of logical object id's-a logical abstraction of the implementational concept of physical object identity [46Г 3]. Since this paper emphasizes logic $\mathrm{T}$ the term "object id" (abbr.Toid) will be used for logical id's only.

Objects represented by "complex" id-terms $\Gamma$ such as addr(13, mainstreet, anywhere) $\Gamma$ usually arise when a complex object (or a class) is constructed out of simpler components $\Gamma$ e.g. $13 \Gamma$ mainstreet Tand anywhere in this example.

It follows from the definition that every F-logic language is uniquely determined by its set of object constructors $\Gamma \mathcal{F}$. In this paper $\Gamma \mathcal{F}$ and $U(\mathcal{F})$ will henceforth denote the set of function symbols and ground terms $\Gamma$ respectively $\Gamma$ where the language $\Gamma \mathcal{L} \Gamma$ will be known from the context.

\section{Molecular Formulas}

A language of F-logic consists of a set of formulae constructed out of the alphabet symbols. As in most other logics $\Gamma$ formulas are built out of simpler formulas by using the usual connectives $\neg \Gamma \vee \Gamma$ and $\wedge$ Tand quantifiers $\exists$ and $\forall$. The simplest kind of formulas are called molecular F-formulas (abbr. $F$-molecules or just molecules).

\footnotetext{
${ }^{5}$ Following [30], first-orderness here means that variables do not range over complex domains, such as domains of sets or functions, but they can range over the intensions of those higher-order structures.
} 
say that plus is a method that returns an integer when invoked on an integer-object with an integerargument. HoweverTwhen plus is invoked on an object in class real with an argument that also comes from

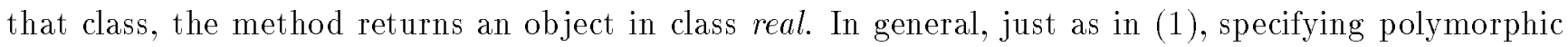
types requires more than one signature. Several interesting examples of polymorphic types (including parameterized types) will be given in Section 11.3.

A form of polymorphism when a method can be invoked with varying number of arguments is called arity-polymorphism. For instance $\Gamma$ student [avgGrade $\Rightarrow$ grade] and student[avgGrade@year $\Rightarrow$ grade] may both be legal invocations (sayTthe first implicitly assuming the current year).

Arity-polymorphism is very popular in logic programming languagesГsuch as Prolog. HoweverTunlike Prolog Tarity polymorphism in F-logic is controlled via signaturesTjust as any other kind of polymorphism. This means that to be able to invoke a method with any given number of arguments $\Gamma$ an appropriate

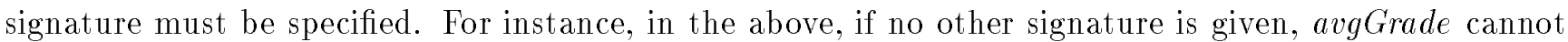
be invoked with more than one argument. Another way to control arity-polymorphism is by turning F-logic into a sorted language. This extension is described in Section 16.

One more kind of polymorphism arises when a method name is declared to be both set-valued and scalar. For instanceTsuppose the following types are defined:

$$
\text { student }[\text { grade } @ \text { course } \Rightarrow \text { integer }] \quad \text { student }[\text { grade @ course } \Rightarrow \text { integer }]
$$

In the first case grade is a set-valued method of one argument that for any student and any given course returns the set of this student's scores in the course (sayTthe scores for all projects and examinations). In the second caseTonly the overall grade for the course is returned. For instance $\mathrm{if}$ sally is a student $\Gamma$ the query

$$
?-\operatorname{sally}[\text { grade@ } @ \rightarrow X]
$$

will return the set of Sally's scores in the database course while the query

$$
?-\operatorname{sally}[\text { grade } @ d b \rightarrow X]
$$

will return the final grade. This kind of polymorphism is controlled as beforeTvia signatures; it can also be controlled via sortsTsimilarly to arity-polymorphism.

Observe that F-logic manipulates several higher-order concepts. In Figure 4Tthe attribute friends is

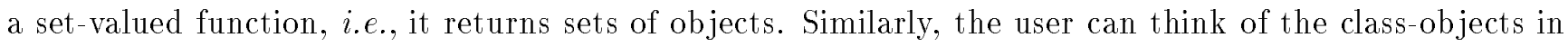
the IS-A hierarchy of Figure 2 as sets ordered by the subset relation.

Furthermore Tattributes and methods are also viewed as objects. ThisTfor instance $i m p l i e s$ that their names can be returned as query answers. In this wayTschema information is turned into data so that it can be manipulated in the same language. This is useful for tasks that require schema exploration in databases (Section 11.4.2) Tinheritance with overriding (Section 14.3) Tand for many other applications (Sections 11.4.3 and 11.4.5). 


\section{Other Features}

Before bidding farewell to this example Twe would like to highlight some other features of F-logic. Suppose that in Statement (i) we replace mngr $\rightarrow b o b$ with mngr $\rightarrow$ phil. Then we would have had a type error. Indeed $\Gamma$ on the one hand $\Gamma$ the deductive rule (vii) implies bob[boss $\rightarrow$ phil]; on the other hand $\Gamma$ from Figure $2 \Gamma$ phil is neither a faculty nor a manager. This contradicts the typing of the attribute boss in (iii). ${ }^{3}$ As will be seen in Section 12 Tthe notions of well-typing and type error have precise model-theoretic meaning in F-logic.

Another aspect of the type system of F-logic is that signature declarations are enforced. For instance $\Gamma$ the following methods are declared in Figure 4 for the members of class faculty:

- name $\mathrm{T}$ friendsTand children-the methods inherited from person;

- worksFor and jointWorks-methods inherited from empl; and

- bossTageThighestDegreeTand papers - the methods directly specified for the class faculty.

Enforcing signature declarations means that these are the only methods applicable to the members of class faculty; any other method is illegal in the scope of that class. Moreover Tit is a type error to invoke the method jointWorks with an argument that is not a member of class empl. Similarly it would be a type error to invoke the methods in the last group on objects that represent employees who are not facultyГor to invoke worksFor or joint Works on objects that are not members of class empl.

In our exampleTinvocations of all methodsTexcept avgSalary Tare sanctioned by signatures declared for appropriate classes. The method avgSalary Ton the other hand is not covered by any signature $\mathrm{which}$ is a violation of the well-typing conditions (to be discussed in Section 12). To correct the problem $\Gamma$ the class faculty (in its role as an object) has to be made into a member of another class 5 say employmentGroup (whose members are various categories of employees $\Gamma$ such as empl $\Gamma$ faculty $\Gamma$ and manager). To give avgSalary a proper typeTwe could then declare employmentGroup[avgSalary $\Rightarrow$ integer $]$.

Observe a difference in the treatment of the non-inheritable method avgSalary (and of all other noninheritable methods in the example) and of the inheritable method highestDegree. The former must be covered by a signature declared for a class where faculty is a member Twhile the latter should be covered by a signature declared for a class where faculty is a subclass. ${ }^{4}$ This is because the non-inheritable method avgSalary is a property of the object faculty itselfT while the inheritable method highestDegree is effectively a property of the objects that are members of the class faculty.

Yet another important aspect of F-logic type system is polymorphism. This means that methods can be invoked on different classes with arguments of different types. For instanceTthe following signatures

$$
\text { integer[plus@integer } \Rightarrow \text { integer }] \quad \text { real[plus@real } \Rightarrow \text { real }]
$$

\footnotetext{
${ }^{3}$ Note that phil is an employee and thus bob[boss $\rightarrow$ phil] complies with the typing of boss for the class empl, as specified in (v). However, bob is also a member of class faculty, and (iii) imposes a stricter type on the attribute boss in that class, requiring phil to be a member of both faculty and manager.

${ }^{4}$ This class can be faculty itself, since the subclass relationship in F-logic is non-strict.
} 
friends $\rightarrow\{b o b\}$. However $\Gamma$ in friends $\rightarrow\{b o b$, sally $\}$ of Statement (ii) and in assistants $\rightarrow$

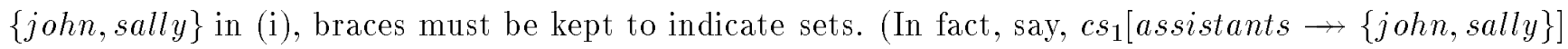
is equivalent to a conjunction of $c s_{1}[$ assistants $\rightarrow j o h n]$ and $c s_{1}[$ assistants $\rightarrow$ sally] Tbut the use of braces leads to a shorter notation).

Statement (vii) is a deductive rule that defines a new attribute $\mathrm{b} b s s \Gamma$ for objects of class empl. It says that an employee's boss is the manager of the department the employee works in. Here we follow the standard convention in logic programming that requires names of logical variables to begin with a capital letter. Statement (viii) defines a method jointWorks whose signature is given in (v). For any object in class person $\Gamma$ jointWorks is a function that takes an object of type person and returns a set of objects of type report; each object in this set represents a paper co-authored by the two people. InformallyI this rule should be read as follows: If a report-object $Z$ Z simultaneously belongs to the sets X.papers and Y.papers $\mathrm{I}^{2}$ where $X$ and $Y$ are faculty-objects $\Gamma$ then $Z$ must also belong to the set returned by the method jointWorks when invoked on the host-object $X$ with the argument $Y$.

It is important to realize here that the variable $Z$ ranges over the members of the set X.papers $\cap$ Y.papers - it is not instantiated to the set itself. Note also that we do not need the restriction $Z$ : report in the rule body because $\Gamma$ for each faculty $\Gamma$ the attribute papers specifies a set of article-objects (by Statement (iii)) $\Gamma$ and article is a subclass of report (see Figure 2). However $\Gamma$ if we were interested in JACM papers onlyTthen the restriction $Z:$ jacm would have been necessary in the body of (viii).

Two uses of the method jointWorks are shown in (x) and (xi). Query (x) asks about the co-authors of mary's paperГjacm 90 Twhile (xi) requests all joint papers that mary co-authors with phil. Query (ix) inquires about all middle-aged employees working for "CS" departments. In particularTfor every such employeeT the attributes boss Tage Tand worksFor are requested. The expected answer to (ix) is:

(xii) $\quad b o b\left[b o s s \rightarrow b o b ;\right.$ age $\rightarrow 40 ;$ worksFor $\left.\rightarrow c s_{1}\right]$.

The object mary does not qualify as an answer to query (ix) because of the unknown age.

In this connection Twe would like to mention the fact that attributes and methods are partial functions. They may be defined on some objects in a class and undefined on another. F-logic distinguishes two reasons for undefinedness: 1) the attribute (or method) may be inapplicable to the object; or 2) it may be applicable but its value is unknown. Case 1) arises due to typing Tas discussed later. Case 2) Ton the other hand Tis essentially a form of a null value which is familiar from database theory.

In the above example and in the rest of this paperTthe term "type" of an object is used interchangeably to refer to classes of that object and to its declared signatures. The correct meaning should be clear from the context. The dual use of this term is appropriate since $\Gamma$ generally the term "type" refers to arbitrary collections of abstract values. Classes and signatures both specify such collections. The former denotes collections of class members which are semantically related objects; the latter denotes collections of objects that are structurally related. Since semantic similarity usually implies structural similarity the two uses of the term "type" are closely related.

\footnotetext{
${ }^{2}$ Here the notation $X$.papers denotes the set of oid's returned by the attribute papers on the object $X$. Similarly for Y.papers.
} 
in $c l[a t t r \Rightarrow(a, b, c)]$ signifies a conjunction of types: the value of attr on any object in class $c l$ must simultaneously belong to each of the classes $a \Gamma b$ Tand $c$. When the set of types to the right of a doubleshafted arrow $\Gamma$ " $\Rightarrow$ " or “ $\Rightarrow$ " $\Gamma$ is a singleton set $\Gamma$ e.g. $\Gamma c[a t t r \Rightarrow(e m p l)] \Gamma$ we shall omit the surrounding parentheses and write $\Gamma$ e.g. $\Gamma c[a t t r \Rightarrow e m p l]$.

Different kinds of information about objects can be mixed in one statement. For instanceTin Statement (iii) Tthe expression highestDegree $\Rightarrow$ degree specifies the type of the attribute highestDegree; the expression highestDegree $\bullet$ phd specifies an inheritable property of faculty; and avgSalary $\rightarrow 50000$ is a non-inheritable property of faculty.

Asserting an inheritable property for a class-object has the effect that every member-object of that

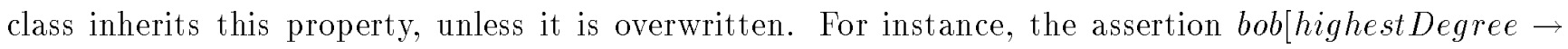
phd] is derivable from (iii) by inheritance. Note that when a property is inherited by a member of the class it becomes non-inheritable; this explains why inheritance derives bob[highestDegree $\rightarrow$ phd] rather than bob[highestDegree $\bullet p h d]$. An inheritable property may also be inherited by a subclass. HoweverTin this case $\Gamma$ the inherited property remains inheritable in this subclass and $\Gamma$ as such $\Gamma i t$ can be passed further down in the hierarchy of objects. For exampleГif lecturer were a subclass of faculty then lecturer $[$ highestDegree $\bullet p h d]$ would be derivable by inheritance [unless lecturer has another inheritable property $(e . g$. . ThighestDegree $\bullet m s)$ that overrides this inheritance. Inheritance will be discussed in detail in Section 14.

In contrast to highestDegree $\bullet$ phd T the property avgSalary $\rightarrow 50000$ in (iii) is not inheritable by

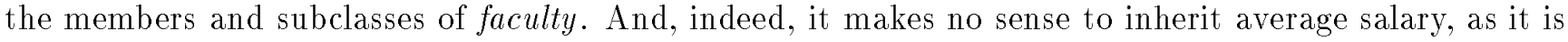
an aggregate property of all members of the class and has no meaning for individual members. Inheriting avgSalary $\rightarrow 50000$ to a subclass is also meaningless $\Gamma$ because subclass members $\Gamma$ e.g. Tall lecturers Tare likely to have a different average salary than all members of the larger class faculty.

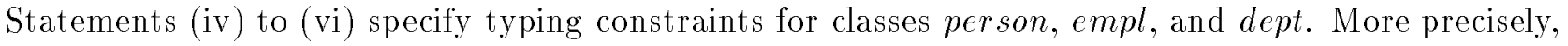
it can be said that these statements define signatures of methods attached to these classes. Two things are worth noting in Statement (iv). First $\Gamma$ the expression jointWorks@person $\Rightarrow$ report describes a method jointWorks Tthat expects one proper argument of type person and returns a set of elements of type report. In object-oriented terms Tthis means that whenever a person-object $\Gamma o b j$ Treceives a messageI jointWorks Twith an argument of class person $\Gamma$ then the reply-message returned by obj will consist of a set of objectsTeach being a member of class report. Note that there is no essential difference between methods and attributes: the latter are simply methods without arguments. Strictly speakingTin Figure 4 we should have written name@ $\Rightarrow$ string and age@ $\rightarrow 40$ instead of name $\Rightarrow$ string and age $\rightarrow$ 40I but the latter short-hand notation is more convenient when no arguments are expected.

The second thing to note in (iv) is the expression children $\Rightarrow$ child(person) $\Gamma$ which specifies a type constraint for the attribute children. Here Child is a unary function symbol and person is a constant denoting a class. The term child(person) Tthen Tis a logical id of another class. Thus Tin F-logic Tfunction symbols are used as constructors of object and class id's.

We shall often omit braces surrounding singleton sets and write $\mathrm{say} \Gamma$ friends $\rightarrow b o b$ instead of 


\section{Database Facts:}

(i) bob $[$ name $\rightarrow$ "Bob"; age $\rightarrow 40$;

worksFor $\rightarrow c s_{1}[$ dname $\rightarrow$ "CS"; mngr $\rightarrow$ bob; assistants $\rightarrow\{$ john, sally $\left.\}]\right]$

(ii) $\operatorname{mary}[$ name $\rightarrow$ "Mary"; highestDegree $\rightarrow$ ms;

$$
\text { friends } \rightarrow\{b o b, \text { sally }\} ; \text { worksFor } \rightarrow c s_{2}[\text { dname } \rightarrow \text { "CS"] ] }
$$

General Class Information:

(iii) faculty[boss $\Rightarrow$ (faculty, manager); age $\Rightarrow$ midaged; highestDegree $\Rightarrow$ degree; $\%$ papers $\Rightarrow$ article; highestDegree $\bullet$ phd; avg Salary $\rightarrow 50000$ ]

(iv) person $[$ name $\Rightarrow$ string; friends $\Rightarrow$ person;

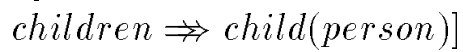

(v) empl[worksFor $\Rightarrow$ dept; boss $\Rightarrow$ empl; jointWorks@empl $\Rightarrow$ report $]$
\% Typing: boss—scalar attribute; returns objects $\% \quad$ belonging to both faculty \& manager $\%$ highestDegree $\bullet$ phd: inheritable property $\%$ avgSalary $\rightarrow 50 \mathrm{~K}$ : non-inheritable property \% Typing: friends - a set-valued attribute \% Typing: each child is in class child(person) \% Typing: employees work for departments \% Typing: jointWorks - a one-argument method

(vi) $\operatorname{dept}[$ assistants $\Rightarrow($ student,empl); mngr $\Rightarrow e m p l] \%$ Typing: assistants are students and employees

\section{Deductive Rules:}

(vii) $E[$ boss $\rightarrow M] \leftarrow E: e m p l \wedge D:$ dept $\wedge E[$ worksFor $\rightarrow D[m n g r \rightarrow M: e m p l]]$

(viii) $X[j$ jointWorks $@ Y \rightarrow Z] \leftarrow Y:$ faculty $\wedge X:$ faculty

$$
\wedge Y[\text { papers } \rightarrow Z] \wedge X[\text { papers } \rightarrow Z]
$$

\section{Queries:}

(ix) $\quad$ ? $-X: e m p l \wedge X[$ boss $\rightarrow Y$; age $\rightarrow Z:$ midaged; worksFor $\rightarrow D[$ dname $\rightarrow$ "CS" $]]$

(x) $\quad ?-\operatorname{mary}[$ jointWorks $@ Y \rightarrow j a c m 90]$

(xi) $\quad ?-\operatorname{mary}[$ jointWorks@phil $\rightarrow Z]$

Figure 4: A Sample Database

In the object-oriented systemsTa method is a function of the form Objects $\times$ Objects ${ }^{n} \longrightarrow$ Objects or Objects $\times$ Objects $^{n} \longrightarrow \mathcal{P}($ Objects $)$ Twhere $\mathcal{P}$ is a power-set operator. Given an object Tits methods are "encapsulated" inside that object and constitute its interface to the rest of the system. The first argument of a method is the object the method is being invoked on-the host object of the invocation. The other arguments are called proper arguments of the invocation.

The reader may have already noticed that double-headed arrows $\Gamma \rightarrow$ and $\Rightarrow$ Tare used in conjunction with set-valued attributes while $\rightarrow \Gamma \Rightarrow$ signify scalar attributes. AlsoГdouble-shafted arrows $\Rightarrow$ and $\Rightarrow \Gamma$ specify types $\Gamma$ while arrows $\rightarrow$ and $\rightarrow$ describe values of attributes. A double-shafted arrow specifies that an attribute (or method) is defined; if it is not given $\Gamma$ the single-shafted arrow may not be used. Given $c l[a t t r \Rightarrow \cdots]$ Tone cannot use obj[attr $\rightarrow \cdots] \Gamma$ where $o b j$ is in class $c l \Gamma$ to give a value to attr (unless attr $\Rightarrow \cdots]$ is specified for some other class where obj also belongs). Also[ note that $(a, b, c)$ 


\begin{tabular}{|c|c|}
\hline empl :: person & yuppie :: young \\
\hline student :: person & 20 : young \\
\hline faculty :: empl & 30 : yappie \\
\hline child(person) :: person & $40:$ midaged \\
\hline john : student & "CS" : string \\
\hline john : empl & "Bob" : string \\
\hline$c s_{1}:$ dept & alice $: \operatorname{child}($ john $)$ \\
\hline
\end{tabular}

Figure 3: F-logic Representation of the IS-A Hierarchy of Figure 2

when viewed as an objectTa class can be a member of another class. For instanceTin Figure 2 Tthe classes string and integer are members of the class datatype.

In the actual syntax of F-logic we use ":" to represent class membership and "::" to denote the subclass relationship. Thus for instance the hierarchy of Figure 2 is recorded as shown in Figure 3. Here a statement such as empl :: person says that empl is a subclass of person; john : empl says that

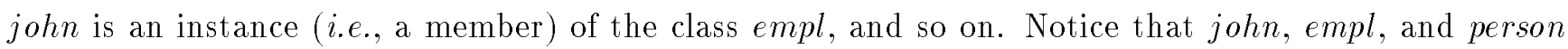
are simply terms that denote objects.

\section{The Object Base}

Figure 4 presents a database fragment describing employees $\Gamma$ students $\Gamma$ and others. The first statement there says that the object $b o b$ is a faculty whose name is " $B o b$ ". Here $b o b$ is a logical id of an object that supposedly represents a person. In contrast $\Gamma$ " $B o b$ " is a member of class string; it represents the value of one of $b o b$ 's attributesTcalled name.

Note that F-logic does not support the dichotomy between "complex objects" and "complex values." We believe that complications introduced by this dichotomy do not justify the benefits (but see Section 15.3). Thus $\Gamma^{\text {" }} B o b$ " is viewed as an oid that represents the string " $B o b$ ".

Statement (i) also says that $b o b$ works in the department denoted via the oid $c s_{1} \Gamma$ the department's name is represented by the oid "CS" Tand its manager is described by the object with oid bob. Note that $b o b$ has cyclic references to itself. Statement (ii) represents similar information about mary. Unlike the attributes name ThighestDegree Tand age Twhich return a single valueTthe attribute friends is set-valued.

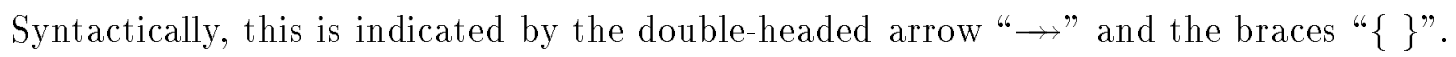

Statements (iii) to (vi) provide general information about classes and their signatures. A signature of a class specifies names of attributes and methods that are applicable to this class $\Gamma$ the type of arguments

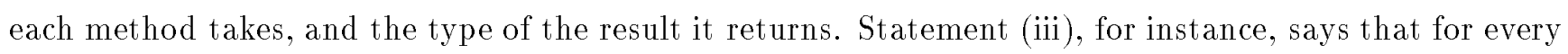
object in class faculty $\Gamma$ the attributes age and highestDegree must be of types midaged and degree $\Gamma$ respectively $\mathrm{T}$ and that the attribute boss returns results that simultaneously belong to classes faculty and manager. 


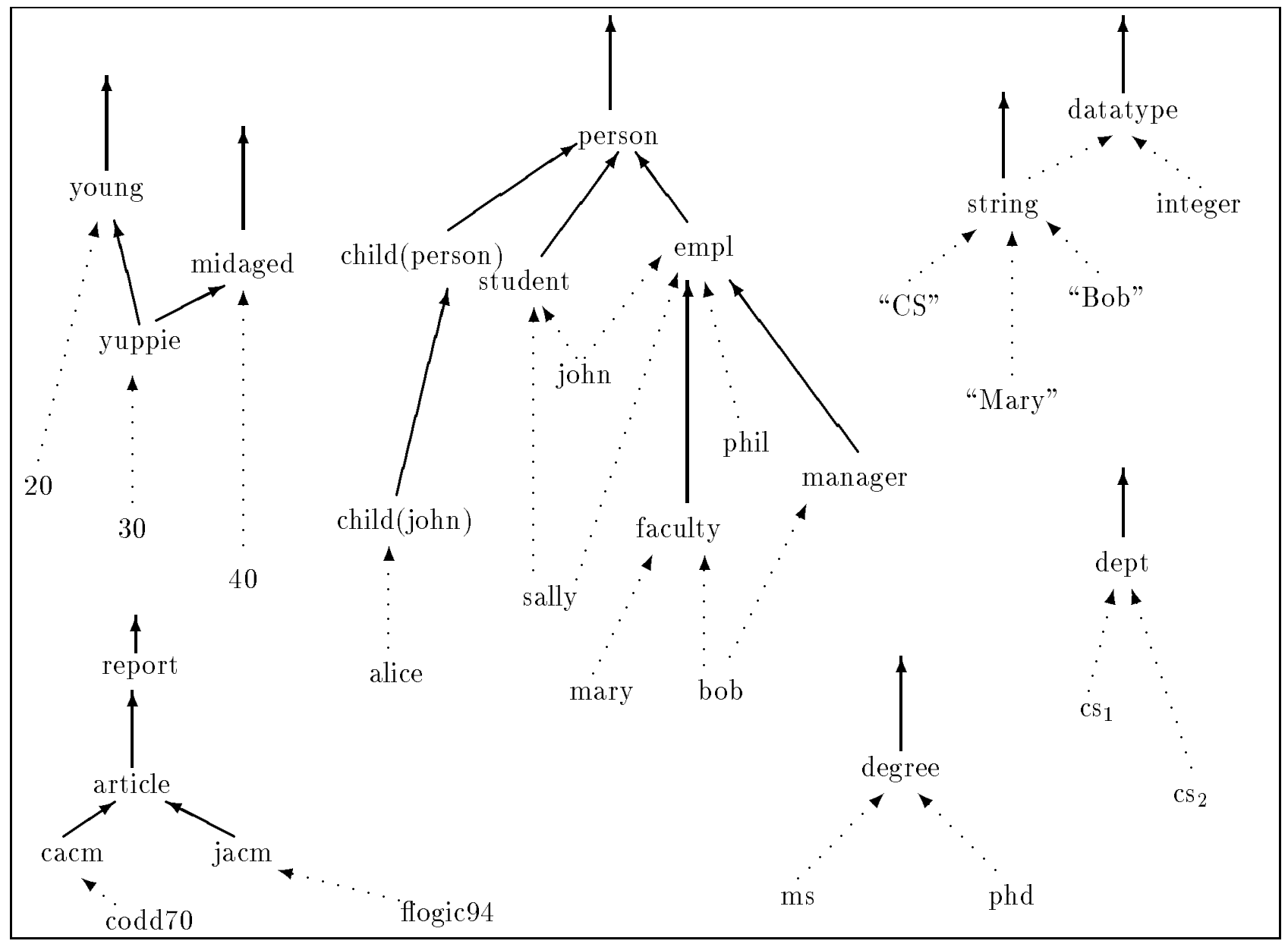

Figure 2: Part of an IS-A Hierarchy

\section{F-logic by Example}

The development of F-logic is guided by the desire to capture in a logically clean way a number of knowledge representation scenarios. Several of the most salient features of F-logic are described in this section by way of an example.

\section{The IS-A Hierarchy}

Figure 2 shows part of a hierarchy of classes and individual objects Twhere solid arcs represent the subclass relationship and dotted arcs represent class membership. This hierarchy asserts that faculty and manager are subclasses of empl; student and empl are subclasses of person; "Mary" and "CS" are members of the class string; and mary is a faculty Twhile sally is a member of the class student. Note that classes are reified Ti.e. Tthey belong to the same domain as individual objects. This endows F-logic with a great deal of uniformity Tmaking it possible to manipulate classes and objects in the same language. In particular $\Gamma$ 


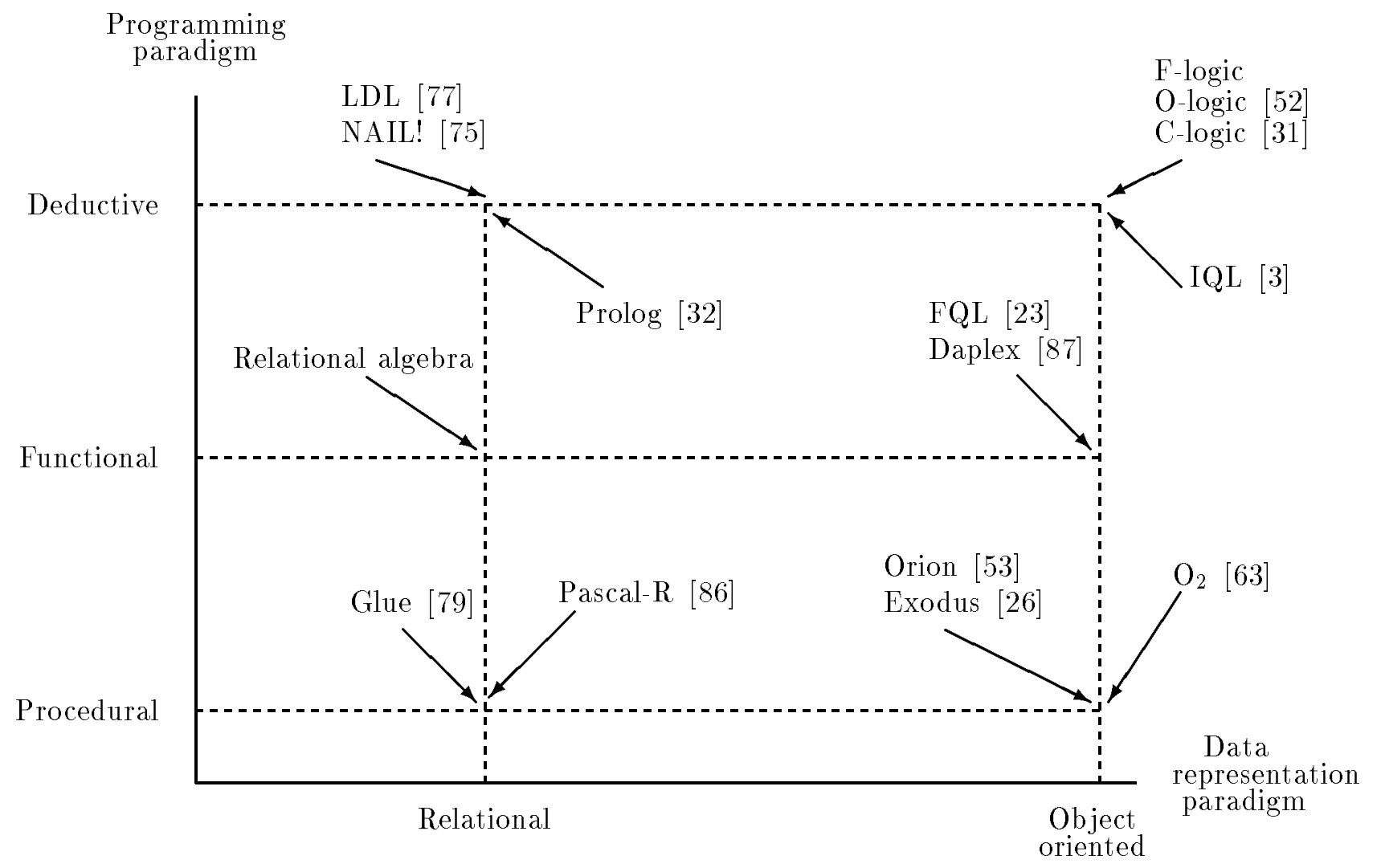

Figure 1: A Classification of Database Programming Languages

We also note that practical considerations sometimes call for a mixture of different paradigms coexisting under the same roof. For instanceГ Prolog [32] has control primitives that give it a procedural

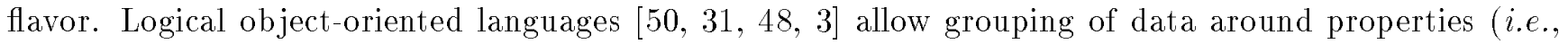
relationally) as well as around objects. IQL [3] relies on a small number of procedural features; Pascal-R [86] offers declarative access to dataTalthough the overall structure of the language is procedural.

In general $\Gamma$ we believe that the future belongs to multi-paradigm languages $\Gamma$ and so the aforementioned "impurity" is not necessarily a drawback. An important research issue is Thowever Tto find ways of clean integration of different kinds of languages. It will be clear from this paper that inside the deductive domain Trelational and object-oriented languages go hand-in-hand. Interfaces between relational and object-oriented (whether declarative or procedural) languages are also known. However $\Gamma$ we are unaware of any clean solution to the integration of procedural object-oriented languages with relational languages; likewise Tthere is no generally agreed upon framework for absorbing functional languages into the deductive paradigm. 
try to put them in a perspective.

It has become customary to define the notion of object-orientation via a long list of properties such systems must have. Some works (e.g.Г[9]) further divide these properties into those that are "mandatory" and those that are "optional." These classifications $\Gamma$ however $\Gamma$ do not explain the difference between relational and object-oriented paradigms; nor do they offer justification for the selection of features that they view as important.

We believe that whether a language has an object-oriented flavor or a relational flavor is primarily determined by the way it represents data. In our view $\mathrm{Tthe}$ central feature of the relational data model is that data is conceptually grouped by properties. For instance Tinformation regarding a given person may be scattered among different relations $\Gamma$ such as employee $\Gamma$ manager $\Gamma$ and project $\Gamma$ each describing different properties attributed to persons. On the other hand $\Gamma$ pieces of data that describe distinct persons via the same property are grouped together in one relation (e.g. Tproject[empId,proj Name]). In contrast Tobject-oriented representation seeks to group data around objects (again $\Gamma$ at the conceptual level). According to this philosophy in language design Tthe user can access-directly or by inheritanceall public information about any object Tonce a "handle" to that object is obtained. This handle is usually referred to as physical object identity - an implementational notion that has a conceptualllanguage-level counterpart Twhich we call logical object identity. Logical oid's were introduced in [50] and subsequently utilized in $[31 \Gamma 15]$ and other works.

The concept of object identity was widely debated in databases. An early attempt to bring this notion into the fold of a logical theory was reported by Maier [65]. Commenting on this work CUllman [93] concluded that the very concept of object identity is incompatible with logic. We feel that both Maier's difficulties and Ullman's scepticism $\Gamma$ result from a confusion that arose due to lumping physical and logical object identities together into a single concept.

Having decided to group data around objectsTit is only natural to try and tap into the potential of such representation by making use of class hierarchies $\Gamma$ inheritance $\Gamma$ typing $\Gamma$ and so on. The difference between "optional" and "mandatory" properties put forward in [9] now becomes a subjective matter of what one perceives to be the most important features of this mode of data representation.

Languages can be classified also along another dimension - their programming paradigm. Here we can point at the following three major groups of languages: proceduralГfunctionalГand deductive. Figure 1 shows how various languages may fit into this framework. Our contention is that the misconception about incompatibility of deductive and object-oriented languages comes from overlooking the fact that the two classification axes of Figure 1 are really orthogonal. What is commonly referred to as "deductive databasesI" is simply a class of languages characterized by the flatTrelational data model and the deductive programming paradigm. In contrast Tmost of the systems that are perceived as "object-oriented" are procedural. Only recently a number of upward-compatible object-oriented logics have been introduced [31Г50Г 48] Which made it clearer how the perceived incompatibility gap could be bridged. The revised version of F-logic presented here continues this line of research $\Gamma$ closing gaps and rectifying flaws of our earlier attempts [50Г48]. 
retain the spirit of object-oriented programming. In contrastTwe propose a logic in which object-oriented concepts are represented directly 5 both syntactically and semantically.

This work builds on our previous papers [50 48$] \Gamma$ which in turn borrowed several important ideas from Maier's O-logic [65] (thatTin its turn $\Gamma$ was inspired by Aït-Kaci's work on $\psi$-terms [6Г5]). In [50] $\Gamma$ we described a logic that adequately covered the structural aspect of complex objects but was short of capturing methods Ttypes $\Gamma$ and inheritance. The earlier version of F-logic reported in [48] was a step towards a higher-order syntax. In particular $\Gamma$ it supported schema exploration and reasoning about structural inheritance. At the same time This version of F-logic had several drawbacks as far as its modeling capabilities were concerned.

One of the problems was that all objects were required to form a lattice $\mathrm{which}$ turned out to be impractical for a logic-based language. Another problem was that semantics of attributes was more appropriate for modeling object types rather than their states. All these problems are rectified in the present paper and Tin addition $\Gamma$ the logic is extended to accommodate types and non-monotonic inheritance.

One aspect of knowledge based systems that is not dealt with here is the issue of updates to database states. Our experience shows that the problem of updates is orthogonal to structural aspects of objectoriented systems $\mathrm{T}$ and this paper deals with this latter issue only. There has been extensive work on formalizing updates within logic. The reader is referred to [19Г20] for a comprehensive discussion of the problem $\Gamma$ for an overview of the related work in the field $\Gamma$ and for solutions to many of the previously outstanding problems.

This paper is organized as follows. Section 2 discusses the differences and the similarities between the object-oriented paradigm and the relational paradigm. Section 3 is an informal introduction to some of the main features of the logic. In Sections $4 \Gamma 5 \Gamma$ and $6 \Gamma$ we describe the syntax and the semantics of F-logic. Section 7 discusses various semantic properties of the logic. Sections 8 Г 9 Г and 10 develop a proof theory for F-logic. Section 11 demonstrates the modeling power of F-logic via a number of nontrivial examples. Section 12 discusses typing. In Section 13 we deal with encapsulation and put forth a novel proposal to view encapsulation as a type-correctness policy. Section 14 presents a semantics for inheritance. An array of issues in data modeling $\Gamma$ such as complex values $\Gamma$ versions control $\Gamma$ and path expressions $\mathrm{T}$ is covered in Section 15. Possible extensions to F-logic are discussed in Section 16. In Section 17 we provide a retrospective view of the internal structure of F-logic and relate it to classical predicate calculus. Section 18 concludes the paper.

\section{A Perspective on Object-Oriented vs. Declarative Programming}

A number of researchers had argued that object-oriented languages are fundamentally different and even incompatible with other paradigms Tespecially with logic programming [93Г67]. The ensuing debate was a reflection of the lack of early success in formalizing many aspects of object-oriented programming. Another reason was that there was no framework in which to classify various approaches $\mathrm{so}$ that their differences and common points could be seen in a perspective. In this section we address these issues and 


\section{Introduction}

The past decade was marked by a considerable interest in the object-oriented approach Tboth within the database community and among researchers in programming languages. Although the very term "the

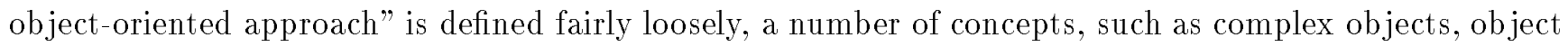

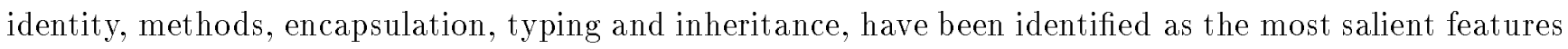
of that approach [12Г $88 \Gamma 103 \Gamma 97 \Gamma 9]$.

One of the important driving forces behind the interest in object-oriented languages in databases is the promise they show in overcoming the so called $\Gamma$ impedance mismatch [66Г103] between programming languages for writing applications and languages for data retrieval. Concurrently $\Gamma$ a different $\Gamma$ deductive approach has gained enormous popularity. Since logic can be used as a computational formalism and as a data specification language proponents of the deductive approach have argued that this approach $\Gamma$ tooTovercomes the aforesaid mismatch problem. However Tin their present form $\Gamma$ both approaches have shortcomings. One of the main problems with the object-oriented approach is the lack of logical semantics $\Gamma$ which traditionally was important for database programming languages. On the other handTdeductive databases normally use a flat data model and do not support data abstraction. It therefore can be expected that combining the two paradigms will pay off in a big way.

A great number of attempts to combine the two approaches has been reported in the literature (see $\Gamma$ e.g.Г[1Г2Г3Г13Г16Г17Г31Г50Г60Г58Г65Г85Г10]) butTin our opinion Tnone was entirely successfully. These approaches would seriously restrict object structure and queries; or they may sacrifice declarativeness by adding extra-logical concepts; or they would not address certain important aspects of object-oriented systems $\Gamma$ such as typing or inheritance.

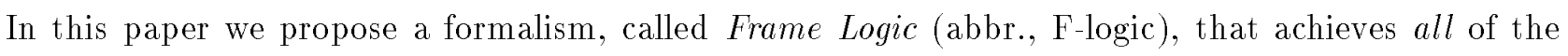
goals listed above and $\Gamma$ in addition $\Gamma$ is suitable for defining and manipulating database schema and types. F-logic is a full-fledged logic; it has a model-theoretic semantics and a sound and complete proof theory. In a senseГF-logic stands in the same relationship to the object-oriented paradigm as classical predicate calculus stands to relational programming.

Besides object-oriented databasesTanother important application for F-logic is in the area of framebased languages in AI [37Г 72] $\Gamma$ since these languages are also built around the concepts of complex objectsTinheritanceTand deduction. It is from this connection that the name "Frame Logic" was derived. HoweverTmost of our terminology comes from the object-oriented parlanceГnot from AI. ThusTwe will be talking about objects and attributes instead of framesTslotsTand the like.

To reason about inheritance and for tasks requiring exploration of the knowledge base schema $\mathrm{\Gamma}$ a logic-based language would be greatly aided by higher-order features. HoweverThigher-order logics must

be approached with great care to ensure the desired computational properties. In the past $\Gamma$ a number of researchers suggested that many useful higher-order concepts of knowledge representation languages can be encoded in predicate calculus [41Г69]. From a programmer's point of view $\Gamma$ however Tencoding does not adequately capture many higher-order constructsTas it gives no direct semantics and does not 
A Appendix: A Perfect-Model Semantics for F-logic 84

B Appendix: A Unification Algorithm for F-molecules $\quad 88$

$\begin{array}{lr}\text { References } & 90\end{array}$ 
11.1 Logic Programs and their Semantics . . . . . . . . . . . . . . . . . 42

11.2 Examples of IS-A Hierarchies . . . . . . . . . . . . . . . . . . 45

11.3 Examples of Type Declarations . . . . . . . . . . . . . . . . . . 47

11.4 Examples of Object Bases . . . . . . . . . . . . . . . . . . 47

11.4.1 Set Manipulation . . . . . . . . . . . . . . . . 48

11.4.2 Querying Database Schema ...................... 50

11.4.3 Representation of Analogies . . . . . . . . . . . . . . . 51

11.4 .4 List Manipulation . . . . . . . . . . . . . . . . . . 52

11.4.5 A Relational Algebra Interpreter . . . . . . . . . . . . . . . . 52

12 Well Typed Programs and Type Errors $\quad 53$

$\begin{array}{ll}13 \text { Encapsulation } & 57\end{array}$

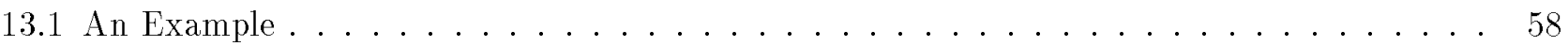

13.2 Modules and Type Correctness . . . . . . . . . . . . . . . . . . 59

14 Inheritance $\quad 62$

14.1 Structural Inheritance . . . . . . . . . . . . . . . . . . . . . . 62

14.2 Behavioral Inheritance . . . . . . . . . . . . . . . . . . . . . . 62

14.2.1 Informal Introduction to the Approach . . . . . . . . . . . . . 65

14.2.2 A Fixpoint Semantics for Non-monotonic Inheritance . . . . . . . . . . . . 66

14.3 Modeling Other Overriding Strategies . . . . . . . . . . . . . . . . 72

15 Other Issues in Data Modeling $\quad 74$

15.1 Existing and Non-existent Objects . . . . . . . . . . . . . . . . . 74

15.2 Empty Sets vs. Undefined Values . . . . . . . . . . . . . . . . . . . . . . 74

15.3 Complex Values. . . . . . . . . . . . . . . . . . . . 75

15.4 Version Control . . . . . . . . . . . . . . . . . 77

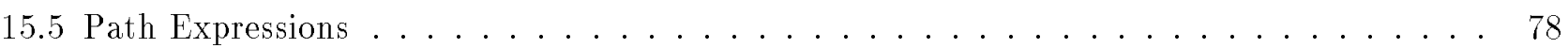

16 Extensions to F-logic $\quad 78$

16.1 Sorted F-logic and its Uses . . . . . . . . . . . . . . . 79

16.2 HiLog-Inspired Extensions . . . . . . . . . . . . . . . . . 80

17 The Anatomy of F-logic $\quad 80$ 


\section{Contents}

1 Introduction 1

2 A Perspective on Object-Oriented vs. Declarative Programming 2

3 F-logic by Example $\quad 5$

4 Syntax $r$

5 Semantics $\quad 16$

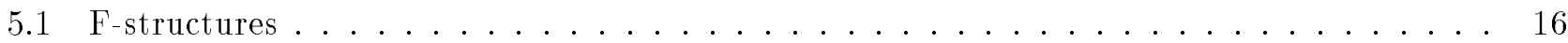

5.2 Satisfaction of F-formulas by F-structures . . . . . . . . . . . . . . . 19

6 Predicates and their Semantics $\quad 21$

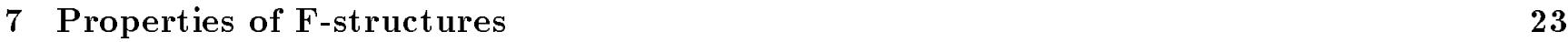

7.1 Properties of the Equality . . . . . . . . . . . . . . . . . 23

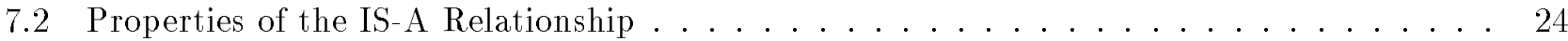

7.3 Properties of Signature Expressions . . . . . . . . . . . . . . . . . . 24

7.4 Miscellaneous Properties . . . . . . . . . . . . . . . . . . 25

8 Herbrand Structures $\quad 26$

9 Skolemization, Clausal Form, and Herbrand's Theorem 28

10 Proof Theory $\quad 31$

10.1 Substitutions and Unifiers . . . . . . . . . . . . . . . . 31

10.2 Core Inference Rules . . . . . . . . . . . . . . . . . . . . 33

10.3 IS-A Inference Rules . . . . . . . . . . . . . . . . . . . . . . . 34

10.4 Type Inference Rules . . . . . . . . . . . . . . . . . . . . . . . . 35

10.5 Miscellaneous Inference Rules . . . . . . . . . . . . . . . . . . . 36

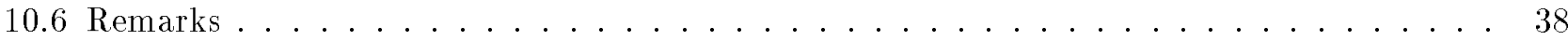

10.7 Soundness of the Proof Theory $\ldots \ldots \ldots \ldots \ldots \ldots \ldots \ldots$

10.8 A Sample Proof . . . . . . . . . . . . . . . . . . . . 39

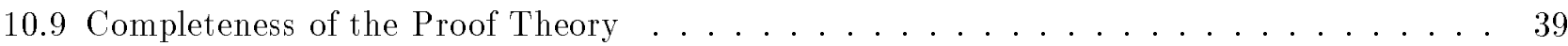




\title{
Logical Foundations of Object-Oriented and Frame-Based Languages
}

\author{
Michael Kifer * Georg Lausen ${ }^{\dagger} \quad$ James $W_{u}^{\ddagger}$
}

\begin{abstract}
We propose a novel logic, called Frame Logic (abbr., F-logic), that accounts in a clean, declarative fashion for most of the structural aspects of object-oriented and frame-based languages. These features include object identity, complex objects, inheritance, polymorphic types, methods, encapsulation, and others. In a sense, F-logic stands in the same relationship to the object-oriented paradigm as classical predicate calculus stands to relational programming. The syntax of F-logic is higher-order, which, among other things, allows the user to explore data and schema using the same declarative language. F-logic has a model-theoretic semantics and a sound and complete resolution-based proof procedure. This paper also discusses various aspects of programming in declarative object-oriented languages based on F-logic.
\end{abstract}

Categories and Subject Descriptors: H.2.1 [Database Management]: Languages-query languages; I.2.3 [Artificial Intelligence]: Deduction and theorem proving-deduction, logic programming, nonmonotonic reasoning; F.4.1 [Mathematical Logic and Formal Languages]: Mathematical logiclogic programming, mechanical theorem proving

General Terms: Languages, Theory

Additional Key Words and Phrases: Object-oriented programming, frame-based languages, deductive databases, logic programming, semantics, proof theory, typing, nonmonotonic inheritance

Technical Report $93 / 06^{1}$

April 1993

Department of Computer Science

SUNY at Stony Brook

Stony Brook NNY 11794

\footnotetext{
*Department of Computer Science, SUNY at Stony Brook, Stony Brook, NY 11794, U.S.A.

Email: kifer@cs.sunysb.edu. Work supported in part by the NSF grants DCR-8603676 and IRI-8903507.

${ }^{\dagger}$ Fakultät für Mathematik und Informatik, Universität Mannheim, D-68131 Mannheim, Germany. Email: lausen@pi3.informatik.uni-mannheim.de.

${ }_{\ddagger}^{\ddagger}$ Renaissance Software, 175 S. San Antonio Rd., Los Altos, CA 94022, U.S.A. Email: wu@rs.com. Work supported in part by the NSF grant IRI-8903507.

${ }^{1}$ A substantial revision of Technical Report 90/14 bearing the same title.
} 